\title{
Evaluation eines Schulinterventionsprojekts zur Steigerung des Obst- und Gemüsekonsums bei Kindern in niedersächsischen Grundschulen
}

\author{
Dissertation \\ zur Erlangung des Doktorgrades \\ der Fakultät Agrarwissenschaften \\ der Georg-August-Universität Göttingen
}

vorgelegt von

Silke Mittmann

geboren in Bünde

Göttingen, im September 2015 
D 7

1. Referent:

PD Dr. med. Thomas Ellrott

2. Korreferentin:

Prof. Dr. Elke Pawelzik

Tag der mündlichen Prüfung: $\quad$ 12. November 2015 


\section{Inhaltsverzeichnis}

Problemstellung / Einleitung $\quad 1$

Theoretischer Bezugsrahmen 3

1. Krebs und Ernährung 3

1.1. Ernährungsassoziierte Erkrankungen 3

1.2. Entstehung von Krebs 6

1.3. Präventionspotential einzelner Lebensmittelinhaltsstoffe $\quad 8$

1.4. Krebs und Prävention 14

2. Ernährungsverhalten und Ernährungsbildung 18

2.1. Obst- und Gemüseverzehr 18

2.2. Obst- und Gemüseverzehr bei Kindern 18

2.3. Entwicklung des Ernährungsverhaltens 20

2.4. Ernährungsbildung im Setting Schule 22

3. Die Gesundheitskampagne ,5-a-day“ 24

3.1. „5 am Tag“ in Deutschland 25

3.2. Projekt „5 am Tag für Kids - Spielend leicht vorbeugen mit Obst \& Gemüse“ 25

Eigene Beiträge

- Behavioural Effects of a Short School-Based Fruit and Vegetable Promotion Programme: 5-a-Day for Kids 39

- Welche Faktoren sind mit dem Obst- und Gemüseverzehr von Grundschülern assoziiert? $\quad 62$

- Praktische Ernährungsbildung in der 5./6. Klasse: Evaluation des Projekts „Entdeckungsreise Essen“

$\begin{array}{ll}\text { Abschlussbetrachtung } & 91\end{array}$

$\begin{array}{ll}\text { Abkürzungsverzeichnis } & 100\end{array}$

$\begin{array}{ll}\text { Danksagung } & 101\end{array}$

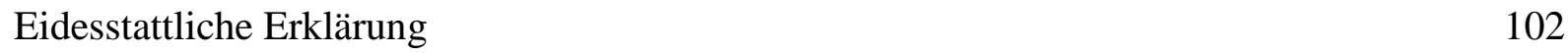




\section{Problemstellung / Einleitung}

Es gibt einen engen Zusammenhang zwischen verschiedenen Nahrungsfaktoren und dem Auftreten von chronischen Krankheiten wie Herz-Kreislauf-Erkrankungen, Diabetes mellitus Typ 2, Adipositas oder bestimmten Krebserkrankungen. Diese Erkrankungen werden auch als nicht-übertragbare Erkrankungen oder Zivilisationserkrankungen bezeichnet.

Ständig verfügbare energiedichte und hochverarbeitete Lebensmittel begünstigen bei gleichzeitigem Bewegungsmangel eine positive Energiebilanz, in deren Folge die oben beschriebenen Erkrankungen auftreten können (Deutsche Adipositas-Gesellschaft, 2014). Derzeit sind beispielsweise knapp ein Viertel aller Erwachsenen in Deutschland morbid adipös (Body Mass Index > $30 \mathrm{~kg} / \mathrm{m}^{2}$ ). Die damit assoziierten Erkrankungen, zu denen HerzKreislauferkrankungen, Diabetes Typ 2, Erkrankungen des Bewegungsapparats, Tumorerkrankungen u.a. gehören, stellen für die Gesellschaft und deren Gesundheitssystem eine erhebliche ökonomische Belastung dar (Deutsche Adipositas-Gesellschaft, 2014).

Neben adäquaten therapeutischen Maßnahmen müssen breit angelegte Präventionsprogramme implementiert werden, um diesen Trend umzukehren. Das Ziel derartiger Präventionsprogramme ist, insbesondere Personengruppen mit hohem Risiko in deren Lebenswelten zu erreichen, und Verhaltensänderungen zu induzieren. Während es zumeist schwierig ist, Risikogruppen wie Familien aus bildungsfernen Schichten im familiären Umfeld zu erreichen, so eröffnet die allgemeine Schulpflicht eine prinzipiell gute Erreichbarkeit im Setting Schule. Daher werden immer mehr präventive Interventionen in sekundären Sozialisationsinstanzen (Kindertageseinrichtungen, Grundschulen, weiterführende Schulen u. a.) angeboten. Auch die Niedersächsische Krebsgesellschaft e.V. offeriert unter dem Namen „5 am Tag für Kids“ ein Präventionsangebot für Dritt- und Viertklässler an niedersächsischen Grundschulen zur Steigerung des Obst- und Gemüseverzehrs (geringe Energiedichte, hoher Mikronährstoff- und Ballaststoffgehalt) u. a. zur Vorbeugung späterer Tumorerkrankungen. Das Präventionskonzept wurde in der vorliegenden Arbeit mit Hilfe einer prospektiven Interventionsstudie auf Akzeptanz und Wirksamkeit hin untersucht.

Im ersten Teil wird das Forschungsprojekt in den theoretischen Bezugsrahmen eingebettet und die Hintergründe des Präventionsansatzes werden erläutert. Dazu werden im ersten Kapitel die Zusammenhänge zwischen Krebs und Ernährung beschrieben. Im Anschluss werden im zweiten Kapitel die Determinanten der Entwicklung des Ernährungsverhaltens im Kindesalter diskutiert. Zudem wird auf den Aspekt der Ernährungsbildung im Setting Schule eingegangen. Die Gesundheitskampagne „,5 am Tag“ und das darauf basierende Projekt „,5 am Tag für Kids“ 
der Niedersächsischen Krebsgesellschaft werden im dritten Kapitel vorgestellt. Die Zeitschriftenbeiträge „Behavioural Effects of a Short School-Based Fruit and Vegetable Promotion Programme: 5-a-Day for Kids" und "Welche Faktoren sind mit dem Obst- und Gemüseverzehr von Grundschülern assoziiert?“ im zweiten Teil der Arbeit thematisieren die Evaluation des Konzeptes. Der Beitrag „Praktische Ernährungsbildung in der 5./6. Klasse: Evaluation des Projekts 'Entdeckungsreise Essen'“، vertieft das Thema Ernährungsbildung und liefert Erkenntnisse, welche Bausteine schulischer Interventionen erfolgsversprechend sind. In der Abschlussbetrachtung werden die Ergebnisse vertiefend und zusammenfassend dargestellt und Limitationen der Arbeit erörtert. 


\section{Theoretischer Bezugsrahmen}

\section{Krebs und Ernährung}

\subsection{Ernährungsassoziierte Erkrankungen}

Eine bedarfsgerechte Ernährung ist die grundlegende Voraussetzung für eine gesunde körperliche, seelische und geistige Entwicklung von Kindern und Jugendlichen und steht in einem engen Zusammenhang mit der Erhaltung von Gesundheit, Wohlbefinden und Leistungsfähigkeit des Menschen (Dixey et al., 2000; Heindl, 2003; Deutsche Gesellschaft für Ernährung e.V., 2008;). Eine angemessene Ernährung und ein körperlich aktiver Lebensstil beinhalten ein großes Präventionspotential im Hinblick auf die dauerhafte Gesunderhaltung der Bevölkerung (Heindl, 2003; Deutsche Gesellschaft für Ernährung e.V., 2008).

Zahlreiche Studien weisen auf den Zusammenhang zwischen Ernährungsdefiziten und der Inzidenz von Herz-Kreislauferkrankungen, bestimmten Krebserkrankungen, Diabetes mellitus Typ 2 oder Adipositas hin (World Cancer Research Fund and American Association for Cancer Research, 1997; Hyson, 2002; 2002; World Health Organization, 2003; Dauchet et al., 2006; World Cancer Research Fund and American Institute for Cancer Research, 2007; Boffetta et al., 2010; Boeing et al., 2012a; Boeing et al., 2012b). Zunehmend sind bereits Kinder und Jugendliche von Übergewicht und Adipositas betroffen. So gelten 15 Prozent der Kinder und Jugendlichen in Deutschland als übergewichtig, davon werden ca. 6 Prozent als adipös eingestuft (Deutsche Gesellschaft für Ernährung e.V., 2008). Mit Adipositas und Fehlernährung ist wiederum ein deutlich erhöhtes Risiko für viele Komorbiditäten verbunden (Deutsche Gesellschaft für Ernährung e.V., 2008).

\section{Obst und Gemüse und Krebs}

Für den zuvor beschriebenen Zusammenhang spielt insbesondere der Einfluss des Obst- und Gemüseverzehrs im Hinblick auf die Prävention ernährungsassoziierter Krankheiten eine bedeutende Rolle und ist daher Untersuchungsgegenstand vieler Studien. Die Deutsche Gesellschaft für Ernährung e.V. (DGE) fasst die Evidenz hinsichtlich des Zusammenhangs zwischen dem Konsum von Obst und Gemüse und dem Auftreten einzelner chronischer Erkrankungen zusammen (Tabelle 1). 
Tabelle 1: Zusammenfassung der Beweislage zum Zusammenhang zwischen Gemüse- und Obstverzehr und der Prävention ausgewählter chronischer Krankheiten

\begin{tabular}{|c|c|c|c|c|}
\hline & \multicolumn{4}{|c|}{ Evidenzbewertung (Härtegrade) } \\
\hline & überzeugend & wahrscheinlich & möglich & unzureichend \\
\hline Adipositas & & $\mathrm{o}^{1}$ & $\downarrow^{2}$ & \\
\hline Diabetes mellitus Typ 2 & & o & & \\
\hline Hypertonie & $\downarrow$ & & & \\
\hline KHK & $\downarrow$ & & & \\
\hline Schlaganfall & $\downarrow$ & & & \\
\hline Krebs & & $\downarrow$ & & \\
\hline $\begin{array}{l}\text { Chronisch entzündliche } \\
\text { Darmerkrankungen }\end{array}$ & & & & $\sim$ \\
\hline Rheumatoide Arthritis (RA) & & & $\downarrow$ & \\
\hline $\begin{array}{l}\text { Chronisch obstruktive } \\
\text { Lungenerkrankung (COPD) }\end{array}$ & & & $\downarrow$ & \\
\hline Asthma & & & $\downarrow$ & \\
\hline Osteoporose & & & $\downarrow$ & \\
\hline $\begin{array}{l}\text { Augenerkrankungen } \\
\text { Makuladegeneration } \\
\text { Katarakt } \\
\text { Glaukom } \\
\text { Diabetische Retinopathie }\end{array}$ & & & $\begin{array}{l}\downarrow \\
\downarrow\end{array}$ & $\sim$ \\
\hline Demenz & & & $\downarrow$ & \\
\hline
\end{tabular}

Quelle: Modifizierte Darstellung nach Deutsche Gesellschaft für Ernährung e.V., 2012, S. 35

Für Hypertonie, koronare Herzkrankheiten und Schlaganfall lässt sich aus der vorliegenden Studienlage eine „überzeugende“ Evidenz ableiten, dass eine obst- und gemüsereiche Ernährung das Erkrankungsrisiko reduziert (Boeing et al., 2012a). Gemäß der Studienlage wird der Evidenzgrad für eine Senkung des Krebsrisikos durch einen hohen Verzehr von Obst und Gemüse mit „wahrscheinlich“ angegeben (Boeing et al., 2012a). Ebenso wird mit „wahrscheinlicher“ Evidenz eingestuft, dass ein vermehrter Konsum von Obst und Gemüse eine Zunahme des Körpergewichtes verhindert. Da Übergewicht zugleich der wichtigste Risikofaktor für das Auftreten von Diabetes mellitus Typ 2 darstellt, wird indirekt auch hierfür eine Risikosenkung prognostiziert (Boeing et al., 2012a). Für Rheumatoide Arthritis, Chronisch obstruktive Lungenkrankheit, Asthma, Osteoporose, bestimmte Augenerkrankungen sowie Demenz wird ein möglicher Zusammenhang mit einer vermehrten Aufnahme von Obst und Gemüse diskutiert (Boeing et al., 2012b).

Bezüglich der Stärke des Zusammenhangs zwischen einem reichlichen Verzehr von Obst und Gemüse und der Krebsinzidenz hat sich die Studienlage in den letzten 30 Jahren gewandelt. Zu Beginn der Forschungsaktivitäten deuteten insbesondere Beobachtungsstudien und Fall- 
Kontroll-Studien darauf hin, dass die Ernährung, insbesondere der Konsum pflanzlicher Lebensmittel, einen bedeutenden Effekt auf das Krebsrisiko hat (Key, 2011; Riboli und Norat, 2003). Im weiteren Verlauf wurde diese Assoziation aufgrund der Ergebnisse einiger prospektiver Kohortenstudien abgeschwächt (Riboli und Norat, 2003; Gerhäuser, 2011). So spricht der Report „Ernährung, körperliche Aktivität und Krebsprävention: Eine globale Perspektive“ des World Cancer Research Fund und des American Institute for Cancer Research aus dem Jahr 1997 noch von einer, ,überzeugenden“ Evidenz, dass eine hohe Zufuhr an Obst und Gemüse das Risiko für folgende Krebsarten reduziert: Mund, Rachen, Speiseröhre, Magen, Dickdarm und Rektum sowie Lunge (World Cancer Research Fund und American Institute for Cancer Research, 1997). Bei einer Neuauflage des Überblicks aus dem Jahr 2007 wird diese Aussage auf eine „wahrscheinliche“ Evidenz herabgestuft (World Cancer Research Fund und American Institute for Cancer Research, 2007). Diese Ausführung gilt als „Goldstandard“ für die Beurteilung von Zusammenhängen zwischen Krebsprävention und Ernährung (Gerhäuser, 2011).

In der groß angelegten EPIC-Kohortenstudie (The European Prospective Investigation into Cancer and Nutrition) mit 521.000 Teilnehmern aus zehn verschiedenen Europäischen Ländern, wurden die Beziehungen zwischen der Ernährung, dem Ernährungszustand, dem Lebensstil und Umweltfaktoren und der Inzidenz von Krebs und weiteren chronischen Erkrankungen aktuell untersucht. Das Review von Bradbury kommt zu dem Ergebnis, dass für neun Krebsarten (Magen, Gallenblase, Bauchspeicheldrüse, Gebärmutterhals, Endometrium, Prostata, Nieren, Blase und Lymphome) keine signifikanten Assoziationen zwischen dem Risiko und der Zufuhr von Obst, Gemüse und Ballaststoffen gefunden werden konnten (Bradbury et al., 2014). Der Obstkonsum ist invers assoziiert mit Tumoren im oberen Gastrointestinaltrakt (Mundhöhle, Kehlkopf, Rachen und Speiseröhre) sowie der Lunge (Bradbury et al., 2014; Gonzalez und Riboli, 2010). Ein Risikozusammenhang besteht ebenfalls zwischen der Aufnahme von Obst, Gemüse bzw. Ballaststoffen und Dickdarmkrebs (Gonzalez und Riboli, 2010; van Duijnhoven et al., 2009). Eine Untersuchung der griechischen EPICKohorte deutet darauf hin, dass der Obst- und Gemüseverzehr das Gesamtkrebsrisiko beeinflusst, wenngleich der Zusammenhang schwächer ausfällt als erwartet (Benetou et al., 2008). 
Aufgrund der latenten Entstehung eines Krebsgeschehens, der komplexen Krankheitsentwicklung und der Herausforderung die multifaktoriellen Aspekte der Ernährungsweise und körperlicher Aktivität im Lauf des Lebens zu charakterisieren, gestaltet es sich äußerst schwierig, gesicherte Zusammenhänge zwischen Ernährungsgewohnheiten, Lebensmitteln bzw. deren einzelnen Inhaltsstoffen sowie körperlicher Zusammensetzung und Krebs zu identifizieren (Wiseman, 2008).

Trotz dieser Entwicklungen kommen Wissenschaftler auch heute noch zu dem Schluss, dass nationale Kampagnen, die einen erhöhten Verzehr von Obst und Gemüse propagieren, ihre Berechtigung haben und die gesellschaftlichen Auswirkungen chronischer Erkrankungen in westlichen Ländern positiv beeinflussen (Boeing et al., 2012a).

\subsection{Entstehung von Krebs}

Krebs entsteht aus einigen Zellen, die in ihrer normalen Regulation von Wachstum, Vermehrung (Proliferation), Differenzierung und interzellulären Beziehungen gestört sind Wiseman, 2008). Die Entwicklung eines Tumors durchläuft drei Phasen: die Initiation, die Promotion und die Progression (Dorai und Aggarwal, 2004; Béliveau und Gingras, 2008).

\section{Initiation:}

Die DNS der Zelle wird durch den Kontakt mit einer krebsauslösenden Substanz (z.B. bestimmte Viren, Tabakrauch, industrielle Emissionen oder krebserregende Substanzen aus Lebensmitteln) irreversibel geschädigt und so eine Mutation hervorgerufen. Auch inflammatorische Prozesse oder Tumorpromotoren können Auslöser sein. Diese sogenannten prämalignen oder präkanzerösen Zellen haben das Potenzial zur Tumorbildung (Gescher, 1998; Dorai und Aggarwal, 2004; Béliveau und Gingras, 2008).

\section{Promotion:}

Sofern durch bestehenden Einfluss karzinogener Substanzen oder anderer günstiger Faktoren weitere Mutationen erzeugt werden, führen diese Mutationen zu veränderten Proteinfunktionen, die wiederum eine unkontrollierte Vermehrung der entarteten Zellen erlaubt. Diese Promotion erstreckt sich über einen langen Zeitraum (10+ Jahre) und ist grundsätzlich reversibel (Gescher, 1998; Béliveau und Gingras, 2008). 


\section{Progression:}

In dieser Phase entwickelt die entartete Zelle ihre malignen Eigenschaften und kann nun in Nachbargewebe eindringen und sich in Form von Metastasen im Organismus ausbreiten (1+ Jahre) (Gescher, 1998; Béliveau und Gingras, 2008).

Die Abbildung 1 verdeutlicht den Entstehungsprozess eines Tumors.

Abbildung 1: Die Krebsentwicklung

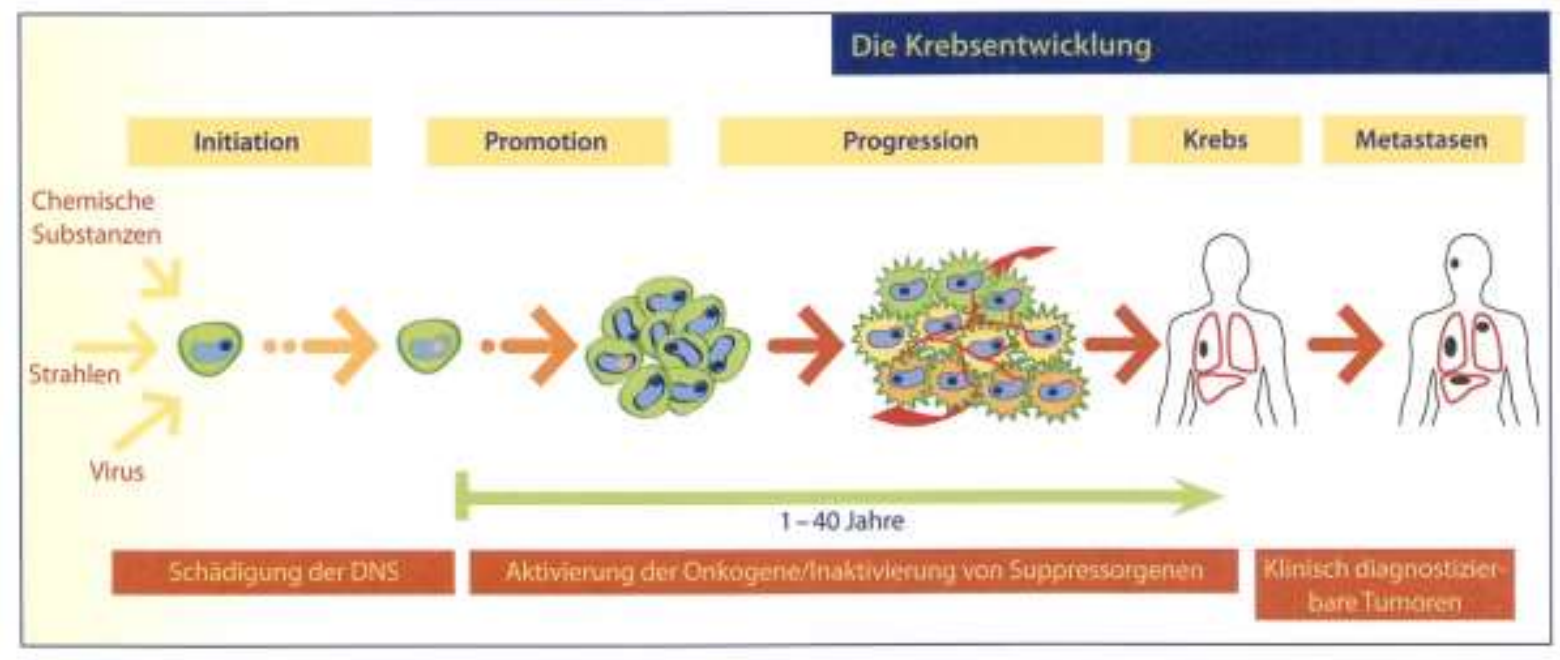

Quelle: Béliveau und Gingras, 2008, S. 37

Jeder Mensch entwickelt im Laufe seines Lebens präkanzeröse Zellveränderungen oder kleinste Tumoren, die in der Regel durch körpereigene Abwehr- bzw. Reparaturmechanismen unschädlich gemacht werden können. Überwinden präkanzeröse Zellen diese Schutzmechanismen jedoch, erwerben sie die Fähigkeit, zu wachsen und in andere Gewebe vorzudringen (Béliveau und Gingras, 2007).

\section{Risikofaktoren}

Doll und Peto schlussfolgerten schon 1981 in ihrem Bericht, dass ungefähr 35 Prozent aller Krebstodesfälle auf die Ernährung zurückzuführen sind (Doll und Peto, 1981). Auch heute noch stellen Ernährungsdefizite sowie Übergewicht bzw. Adipositas und fehlende körperliche Aktivität bedeutende Risikofaktoren für das Auftreten einer Krebserkrankung dar (Eichholzer, 2000; Béliveau und Gingras, 2008; Wiseman, 2008).

Lebensstilfaktoren machen den überwiegenden Teil der Risikofaktoren aus, während genetische Faktoren mit geschätzten 15 Prozent eine nachrangigere Rolle spielen. Die Abbildung 2 kennzeichnet die Risikofaktoren für die Entstehung einer Krebserkrankung. 
Abbildung 2: Risikofaktoren für eine Krebserkrankung

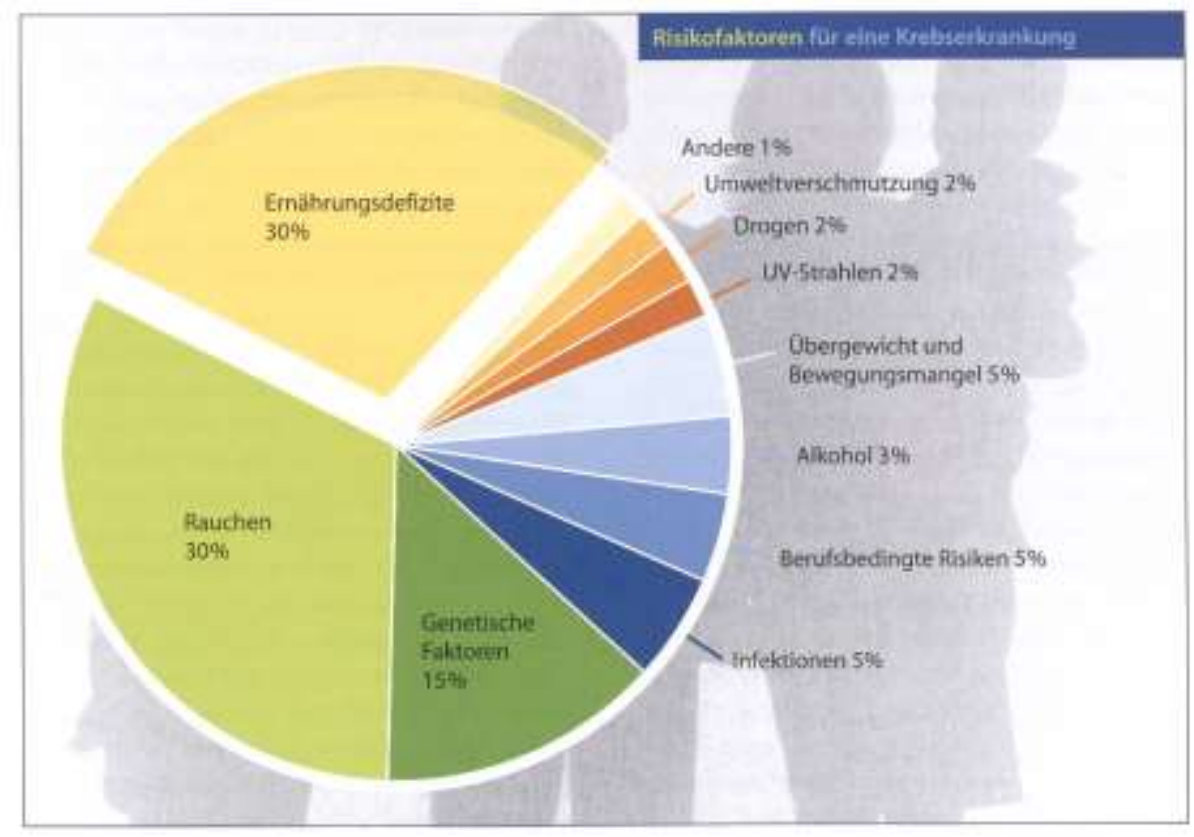

Quelle: Béliveau und Gingras, 2008, S. 19

Zunehmend weisen Studien darauf hin, dass Bestandteile aus pflanzlichen Lebensmitteln in die verschiedenen Phasen der Tumorentstehung eingreifen und so das Wachstum von Mikrotumoren verhindern können (Béliveau und Gingras, 2007). Diese Mechanismen werden im folgenden Kapitel näher beschrieben.

\subsection{Präventionspotential einzelner Lebensmittelinhaltsstoffe}

Obst und Gemüse nehmen bei den Empfehlungen für eine ausgewogene Ernährung einen besonderen Stellenwert ein, da sie nicht nur wichtige Nährstoffe wie Vitamine, Mineralstoffe, sekundäre Pflanzenstoffe sowie Ballaststoffe liefern (Slavin und Lloyd, 2012), sondern diese Nahrungsbestandteile auch über chemopräventive Wirkungen verfügen (Gerhäuser, 2011). Ein weiterer Vorteil ist, dass Obst und Gemüse über eine geringe Energiedichte verfügen, wodurch das Gewichtsmanagement positiv beeinflusst wird. Damit wiederum wird das durch Übergewicht verursachte Risiko für das Auftreten weiterer Komorbiditäten minimiert (Slavin und Lloyd, 2012).

$\mathrm{Zu}$ Beginn der Forschungen wurde vermutet, dass die krebspräventiven Effekte auf einzelne Antioxidantien wie Vitamin A, C und E oder Selen zurückzuführen sind. Heute ist man sich einig, dass der gesundheitliche Nutzen (antioxidative und antikanzerogene Wirkungen) einer obst- und gemüsereichen Ernährung auf additiven und synergistischen Effekten der natürlichen 
Pflanzeninhaltstoffe beruht (Donaldson, 2004; Liu, 2004, Deutsche Gesellschaft für Ernährung e.V., 2004). Aus diesem Grund besteht Konsens darin, dass die Supplementierung mit einzelnen hochdosierten Nährstoffen keinen gesundheitlichen Nutzen verspricht (Deutsche Gesellschaft für Ernährung e.V., 2004; Kushi et al., 2006).

\section{Vitamine}

Vitamin A spielt eine Rolle bei der Proliferation und der Ausdifferenzierung von Epithelzellen sowie der Gesunderhaltung des Gewebes und wird in diesem Kontext mit einem möglichen krebspräventiven Effekt assoziiert (Kushi et al., 2012, Knasmüller et al., 2014). Weitere Mechanismen, die im Hinblick auf die Krebsprävention erforscht werden, sind die antioxidativen Effekte sowie die Auslösung von Apoptose (programmierter Zelltod) und die Tumorsuppression (Knasmüller et al., 2014).

Vitamin E stellt einen hoch antioxidativ-wirksamen Nährstoff dar. Aufgrund dessen wird ein potentieller Einfluss auf die Kanzerogenese untersucht u. a. die Auslösung der Apoptose und die Beeinflussung der Angiogenese (Wachstum von Blutgefäßen) (Kushi et al., 2012, Knasmüller et al., 2014).

Eine Vitamin-C-reiche Ernährung wird mit einer Reduzierung des Krebsrisikos in Verbindung gebracht. Zum einen verhindert Vitamin C oxidative DNA-Schäden, zum anderen werden auch Effekte auf die Angiogenese und Apoptose diskutiert (Knasmüller et al., 2014). In Tierexperimenten konnte festgestellt werden, dass die Salze der Ascorbinsäure in hohen Konzentrationen toxisch auf Krebszellen wirken (Donaldson, 2004).

Randomisierte Kontrollstudien, in denen den Teilnehmern hohe Gaben von Beta-Carotin (Vorstufe von Vitamin A), Vitamin C oder E bzw. Alpha-Tocopherol in Form von Supplementen verabreicht wurden, konnten die prognostizierte Schutzwirkung bzw. Risikosenkung von Krebs nicht belegen (Kushi et al., 2006; Kushi et al., 2012). Im Gegenteil, die Gabe führte teilweise zu einer erhöhten krebsbedingten Sterblichkeitsrate (Béliveau und Gingras, 2008) bzw. zu einem erhöhten Krebsrisiko (Kushi et al., 2012).

Vitamin D ist an zahlreichen krebspräventiven Vorgängen beteiligt: Regulation der Zellteilung, Auslösung von Apoptose, Schutz vor Entzündungen, Auslösung von Differenzierungsvorgängen sowie der Inhibition der Invasion und Metastasierung (Knasmüller et al., 2014). Evidenzbasierte Erkenntnisse lassen vermuten, dass Vitamin D positive Effekte auf Dickdarm- und Brustkrebs aufweist (Kushi et al., 2012, Knasmüller et al., 2014). Das aktiv wirksame Vitamin D-Hormon Calcitriol scheint über antikanzerogene Wirkungen zu verfügen, z.B. verhindert es das Wachstum von bestimmten Körperzellen (Donaldson, 2004). 
Eine krebspräventive Wirkung von Vitamin B12 wird diskutiert, konnte bisher aber noch nicht bewiesen werden. Studien zeigen, dass der Nährstoff eine Rolle bei der genetischen Stabilität und der Reparatur von DNA spielt und damit auch für die Kanzerogenese und die Krebstherapie relevant sein könnte (Donaldson, 2004).

Folat / Folsäure ist an der DNA-Methylierung und der DNA-Synthese beteiligt und ist insbesondere für schnell teilendes Gewebe wichtig (Donaldson, 2004). Ein Mangel an diesem Nährstoff wird in Zusammenhang mit dem Krebsrisiko (Dickdarmkrebs und Brustkrebs) (Knasmüller et al., 2014) und der Kanzerogenese gebracht, da die Gen-Expression beeinflusst wird und vermehrt DNA-Schäden auftreten (Key, 2004). Bisherige Studien deuten darauf hin, dass Folate, die aus Obst, Gemüse und Vollkorn-Getreideprodukten aufgenommen werden, das Risiko möglicherweise senken (World Health Organization, 2003; Kushi et al., 2012, Knasmüller et al., 2014).

\section{Mineralstoffe}

Bei den Mineralstoffen wird u. a. Selen als Stoff mit krebsvorbeugenden Eigenschaften diskutiert. Es spielt bei zahlreichen Vorgängen im Körper eine Rolle:

1. Selen ist beteiligt an Enzymvorgängen, die Redox-Reaktionen katalysieren. Diese Reaktionen können Krebszellen zur Apoptose veranlassen.

2. Selen ist ein Bestandteil eines antioxidativen Enzyms.

3. Im Immunsystem verbessert Selen die Immunabwehr bei Infektionen. Nach Zufuhr von Selen finden sich ein Anstieg von Zytokinen und eine erhöhte Proliferation von T- und T-Helfer-Zellen.

4. Selen induziert möglicherweise die Entgiftung karzinogener Stoffe in der Leber.

5. Selen hat eine hemmende Wirkung auf Prostaglandine, die inflammatorische Prozesse verursachen.

6. Selen hat einen Einfluss auf das Tumorwachstum.

(Donaldson, 2004, Knasmüller et al., 2014)

Es gibt Hinweise aus Untersuchungen, dass Selen das Risiko für das Auftreten bestimmter Tumorarten senken kann (Kushi et al., 2006). Eine hochdosierte Supplementierung wird nicht empfohlen, da Selen auch toxische Wirkungen haben kann, zumal Studien eine Senkung des Krebsrisikos unter Gabe von Selen in Form von Supplementen nicht belegen (Kushi et al., 2012). 
Bezüglich Eisen werden sowohl ein Mangel, als auch ein Überschuss mit einem erhöhten Krebsrisiko (insbesondere für die Organe des Verdauungstraktes) diskutiert. Jedoch ist die Datenlage bisher zu unbefriedigend, um klare Zusammenhänge zu detektieren (Knasmüller et al., 2014).

Zink ist Bestandteil von Transkriptionsfaktoren, DNA-Reparaturproteinen und Tumorsuppressorproteinen. Im Krebsgeschehen werden dem Mineralstoff folgende präventive Wirkungen zugesprochen: Antioxidative Effekte, Beeinflussung der DNA-Reparatur und Beeinflussung des Immunsystems und der Apoptose (Knasmüller et al., 2014).

\section{Sekundäre Pflanzenstoffe}

Man geht davon aus, dass es ungefähr 10.000 sekundäre Pflanzenstoffe gibt. Betrachtet man diese Fülle, sind bisher nur wenige Stoffe und deren Wirkungen erforscht. Den sekundären Pflanzenstoffen werden folgende gesundheitsfördernde Effekte zugesprochen: antikanzerogene, antioxidative, immunmodulatorische, cholesterol-senkende, antibiotische und antithrombotische Wirkungen (Deutsche Gesellschaft für Ernährung e.V., 2004).

Tier- und Laborstudien zeigen, dass zahlreiche sekundäre Pflanzenstoffe in verschiedene zelluläre Prozesse eingreifen und so einen präventiven Effekt sowohl auf die Entstehung von Krebs, als auch einen positiven Einfluss auf das Fortschreiten des Krebsgeschehens haben (Liu, 2004; Béliveau und Gingras, 2007). Beispielsweise können sie oxidative DNA-Schäden, die durch freie Radikale, krebserregende Stoffe aus der Nahrung oder Umwelt oder durch Stoffwechselprodukte hervorgerufen werden, vermindern (Deutsche Gesellschaft für Ernährung e.V., 2004; Béliveau und Gingras, 2007). Zudem können sekundäre Pflanzenstoffe unerwünschtes Zellwachstum verhindern (Dorai und Aggarwal, 2004). Einige Pflanzenstoffe verhindern das Tumorwachstum, indem sie Krebszellen zur Apoptose veranlassen bzw. die Expression von anti-apoptotischen Proteinen unterdrücken (Dorai und Aggarwal, 2004; Liu, 2004). Untersuchungen zeigen, dass einige Inhaltsstoffe aus pflanzlichen Lebensmitteln über anti-angiogene Aktivitäten verfügen, d.h. dass sie die Neubildung von Blutgefäßen, die der Tumor zur eigenen Versorgung mit Sauerstoff und Nährstoffen bildet, unterbinden (Dorai und Aggarwal, 2004; Liu, 2004; Béliveau und Gingras, 2007). Darüber hinaus gibt es Hinweise, dass auch inflammatorische Prozesse beim Fortschreiten eines Krebsgeschehens eine Rolle spielen, die durch anti-inflammatorisch wirksame sekundäre Pflanzenstoffe beeinflusst werden (Dorai und Aggarwal, 2004; Béliveau und Gingras, 2007). 
Die unterschiedlichen Wirkungen von sekundären Pflanzenstoffen im Überblick:

Direkte inhibitorische Wirkungen auf Krebszellen:

$$
\downarrow
$$

Reduzierung von DNA-Schäden

\section{Zytotoxizität gegen-} über Krebszellen

Effekte auf die Mikroumgebung von Tumoren:

\section{$\downarrow$}

Anti-angiogene Möglichkeiten
Anti-inflammatorische Effekte

(eigene Darstellung nach Béliveau und Gingras, 2007)

\section{Ballaststoffe}

Die empfohlene tägliche Zufuhrmenge an Ballaststoffen für einen Erwachsenen beträgt laut DGE mindestens $30 \mathrm{~g}$ (Deutsche Gesellschaft für Ernährung e.V., 2015). Eine hohe Zufuhr an Ballaststoffen hat positive Auswirkungen auf den Energiestoffwechsel und ist mit protektiven Wirkungen vor Diabetes mellitus Typ 2, Herz-Kreislauf-Erkrankungen sowie Dickdarm- und Brustkrebs assoziiert (Kushi, 2006; Slavin und Lloyd, 2012, Kushi et al., 2012; Knasmüller et al., 2014). Diesen schützenden Wirkungen liegen folgende Effekte zugrunde:

- Wasserunlösliche Ballaststoffe vergrößern das Stuhlvolumen und beschleunigen die Darmpassage, so dass die Kontaktzeit krebserregender Stoffe mit der Darmwand reduziert wird (Slavin und Lloyd, 2012, Knasmüller et al., 2014),

- Ballaststoffe binden und inaktivieren Kanzerogene (Knasmüller et al., 2014),

- Ballaststoffe werden größtenteils zu kurzkettigen Fettsäuren abgebaut, die schützende Effekte aufweisen (Knasmüller et al., 2014),

- Wasserlösliche Ballaststoffe beeinflussen Serumlipide positiv und vermindern so das Risiko für kardiovaskuläre Erkrankungen (Kushi et al., 2006),

- Ballaststoffe beeinflussen den Hormonstatus (Östrogenspiegel) positiv (Knasmüller et al., 2014)

Die nachfolgende Abbildung 3 verdeutlicht die Mechanismen der Ballaststoffe. 
Abbildung 3: Mechanismen des Krebsschutzes durch Ballaststoffe $\downarrow$ Abnahme

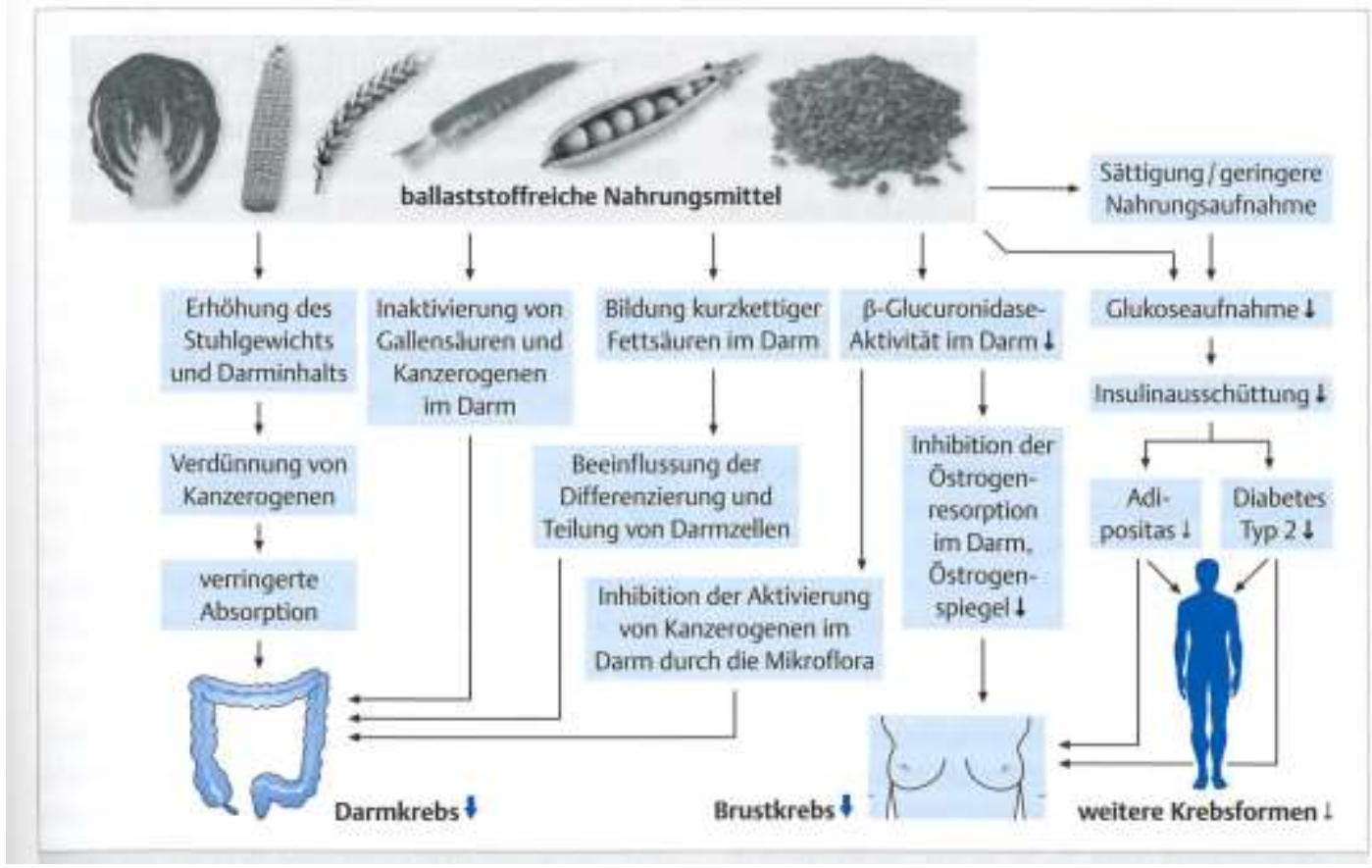

Quelle: Knasmüller et al., 2014, S. 267

An dieser Stelle kann nur ein Auszug der bisherigen Studienlage abgebildet werden. Insgesamt betrachtet, sind die Zusammenhänge zwischen einzelnen Lebensmittelinhaltsstoffen und ihren Wirkungen im Körper bzw. ihrer Beteiligung an einem Krebsgeschehen zum Teil noch zu unzureichend untersucht, um daraus eindeutige Rückschlüsse ziehen zu können und um konkrete Handlungsempfehlungen abzuleiten (Deutsche Gesellschaft für Ernährung e.V., 2004). Darüber hinaus gestaltet es sich schwierig, Ergebnisse aus Tier- oder Laborstudien, die einzelne Nährstoffe und deren Stoffwechselprozesse untersuchen, auf die Bevölkerung zu übertragen, da die Ernährung des Menschen einen individuellen, multifaktoriellen und komplexen Prozess darstellt (Kushi et al., 2012). 


\subsection{Krebs und Prävention}

Im Jahr 2010 sind nach Schätzung der Gesellschaft epidemiologischer Krebsregister in Deutschland und des Robert Koch-Instituts (RKI) ungefähr 477.300 Menschen in Deutschland neu an Krebs erkrankt (Robert Koch-Institut und Gesellschaft der epidemiologischen Krebsregister in Deutschland e.V., 2013). In Niedersachsen traten im Jahr 2011 insgesamt 47.779 Neuerkrankungen auf (Registerstelle des EKN, 2014). Im Jahr 2012 starben laut Angaben des statistischen Bundesamtes 869.582 Menschen. Dabei gingen fast mehr als zwei Drittel aller Sterbefälle auf Krankheiten des Kreislaufsystems oder auf (bösartige) Neubildungen zurück (Statistisches Bundesamt, Todesursachen, 2014). Die Krankheitskosten für bösartige Neubildungen beliefen sich in 2008 auf 15.466 Millionen Euro (Statistisches Bundesamt, Krankheitskosten, 2014). In einer Studie wurden die durch Krebs verursachten Kosten für die gesamte Europäische Union berechnet. Für Deutschland wurden hier für das Jahr 2009 Kosten in Höhe von 14.893 Millionen Euro veranschlagt (Luengo-Fernandez et al., 2013). In diesem Kontext muss jedoch auch erwähnt werden, dass nicht nur die gesamtgesellschaftlichen und wirtschaftlichen Konsequenzen bei der Betrachtung zu berücksichtigen sind, sondern auch die Tatsache, dass hinter jeder Krebserkrankung ein persönliches Schicksal steht, dass oft mit enormem Leid, Arbeitslosigkeit und / oder finanzieller Notlage verbunden ist (Eyre et al., 2004). Vor diesem Hintergrund ist es besonders wichtig, den Stellenwert von (Primär-)Prävention hervorzuheben, um die finanziellen, sozialen und persönlichen Auswirkungen von Krebserkrankungen zu mindern (Wiseman, 2008).

Unter Prävention werden grundsätzlich alle Ansätze zusammengefasst, die darauf abzielen, das Auftreten einer Krankheit zu vermeiden, indem die Auslösefaktoren zurückgedrängt bzw. ausgeschaltet werden (Hurrelmann et al., 2010). Hierbei wird zwischen Primär-, Sekundär- und Tertiärprävention unterschieden.

Primärprävention interveniert frühzeitig und hat zum Ziel, die Entstehung einer Krankheit im Vorfeld zu verhindern (Österreichische Akademie für Arbeitsmedizin und Prävention, 2014). Zwei Basisstrategien sind hier die Verhaltensprävention und die Verhältnisprävention. Bei der Verhaltensprävention geht es darum, dass Individuum $\mathrm{zu}$ einem gesundheitsförderlichen Verhalten $\mathrm{zu}$ befähigen und $\mathrm{zu}$ motivieren (Bayrisches Landesamt für Gesundheit und Lebensmittelsicherheit, 2010). Beispiele hierfür sind erzieherische, bildende oder beratende Maßnahmen zu Themen wie Ernährung, Bewegung oder Sucht. Die gesundheitsförderliche Gestaltung der verschiedenen Lebensbereiche wie Schule, Arbeit oder Freizeit steht im Fokus der Verhältnisprävention (Slesina, 2007). 
Sekundärprävention setzt im Frühstadium einer Krankheit an. Sie dient zur Früherkennung von Krankheiten und der Eindämmung ihres Fortschreitens (Progredienz) oder der Chronifizierung einer Erkrankung. Beispiele für Sekundärprävention sind Vorsorge- und Früherkennungsuntersuchungen wie das Brustkrebs-Screening oder die Darmspiegelung (Slesina, 2007; Österreichische Akademie für Arbeitsmedizin und Prävention, 2014).

Tertiärprävention findet nach einer Akutbehandlung oder der Manifestation einer Erkrankung statt. Auf diesem Weg sollen Folgeschäden und Rückfälle verhindert werden (Slesina, 2007; Österreichische Akademie für Arbeitsmedizin und Prävention, 2014).

Im Gegensatz dazu soll Menschen bei der Gesundheitsförderung durch die Verbesserung ihrer Lebensbedingungen eine Stärkung der gesundheitlichen Ressourcen ermöglicht werden (Klotz, et al., 2006; Hurrelmann et al., 2010). Beide Begriffe - Gesundheitsförderung und Prävention beinhalten Formen der Intervention, d. h. „das gezielte Eingreifen von Akteuren, meist öffentlich und / oder professionell autorisierter Personen und Institutionen, um sich abzeichnende oder bereits eingetretene Verschlechterungen der Gesundheit in relevanten Bevölkerungsgruppen zu beeinflussen“ (Hurrelmann et al., 2010, S. 14). Präventive Interventionen identifizieren die krankheitsauslösenden Risikofaktoren und versuchen frühzeitig in die Entwicklung einzugreifen (Klotz et al., 2006; Hurrelmann et al., 2010). Die Risikofaktoren lassen sich in drei Gruppen einteilen:

- Genetische, physiologische und psychische Dispositionen

- Behaviorale Dispositionen und Verhaltensweisen wie Rauchen, fettreiche Ernährung, wenig Bewegung

- Umweltbezogene individuelle Dispositionen wie Strahlenbelastung, erhöhte Sonnenexposition

(Klotz et al., 2006; Hurrelmann et al., 2010)

Hurrelmann et al. kritisieren in diesem Zusammenhang, dass die gesundheitlichen Versorgungssysteme den Schwerpunkt auf die Kuration und Therapie von Krankheiten legen (Hurrelmann et al., 2010). Den geringen Stellenwert von Prävention und Gesundheitsförderung im deutschen Gesundheitssystem machen die Herausgeber an der Verteilung der finanziellen Ressourcen und den gesetzlichen Rahmenbedingungen fest (Hurrelmann et al., 2010). Demnach werden nur vier Prozent des Gesamtbudgets der gesetzlichen Krankenversicherungen in präventive Maßnahmen investiert (Hurrelmann et al., 2010), davon ein Prozent für Primärprävention (Willich, 2007). Im Berichtsjahr 2013 wurden für die Prävention und die 
betriebliche Gesundheitsförderung insgesamt 267 Millionen Euro ausgegeben (Medizinischer Dienst des Spitzenverbandes Bund der Krankenkassen e.V. und GKV-Spitzenverband, 2014). Das entspricht 3,82 Euro pro Versichertem (Medizinischer Dienst des Spitzenverbandes Bund der Krankenkassen e.V. und GKV-Spitzenverband, 2014).

Laut einem Gutachten des Sachverständigenrates für die Konzertierte Aktion im Gesundheitswesen ließen sich theoretisch die Gesundheitsausgaben in Deutschland um 25-30 Prozent durch langfristige Prävention reduzieren (Sachverständigenrat für die Konzertierte Aktion im Gesundheitswesen, 2000/2001). Die Abbildung 4 verdeutlicht graphisch das Potential der Kosteneinsparung.

Abbildung 4: Modellhafte Kosten-Nutzen-Betrachtung

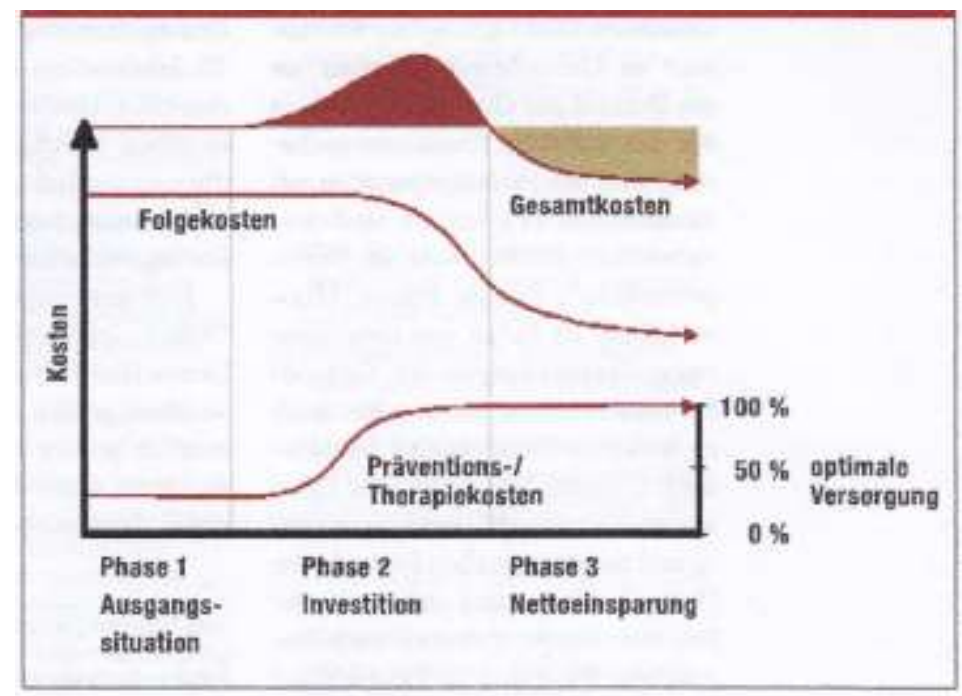

Quelle: Willich, 2007, S. A 1894

Da die Entstehung von Krebs weitestgehend von Umweltfaktoren wie Ernährung, Konsum von Genussmitteln wie Alkohol und Tabak sowie körperlicher Aktivität beeinflusst wird, bietet eine Verbesserung dieser Faktoren ein nicht zu unterschätzendes Präventionspotential (Eichholzer, 2000). Ein gesünderer Lebensstil kann dazu beitragen, das individuelle Krebsrisiko zu senken. Die entsprechenden Grundlagen für einen gesundheitsförderlichen Lebensstil sollten bereits im Kindesalter gelegt werden, da einmal erworbene Gewohnheiten häufig auch im Erwachsenenalter Bestand haben (Burchett, 2003).

Auf Basis der bisherigen wissenschaftlichen Erkenntnisse, hat die Association of European Cancer Leagues Empfehlungen, den sogenannten Europäischen Kodex gegen Krebs, herausgegeben. 
Die Einhaltung der nachfolgenden Regeln soll zur Krebsprävention beitragen:

Regel 1: Rauchen Sie nicht. Verwenden Sie keine Art von Tabak (z.B. Kau- oder Schnupftabak).

Regel 2: $\quad$ Halten Sie Ihre Wohnung und Ihr Arbeitsumfeld rauchfrei.

Regel 3: Halten Sie ein gesundes Körpergewicht.

Regel 4: Betätigen Sie sich täglich körperlich aktiv. Reduzieren Sie sitzende Tätigkeiten. Regel 5: Ernähren Sie sich ausgewogen und gesund: Essen Sie viel Getreide, Hülsenfrüchte sowie Obst und Gemüse. Reduzieren Sie den Konsum von hochkalorischen Lebensmitteln und zuckerhaltigen Getränken. Vermeiden Sie verarbeitetes Fleisch und reduzieren Sie die Zufuhr von rotem Fleisch und salzhaltigen Speisen.

Regel 6: Verringern Sie Ihren Alkoholkonsum.

Regel 7: Vorsicht vor übermäßiger Sonnenbestrahlung. Dies gilt vor allem für Kinder, weil sie eine besonders empfindliche Haut haben.

Regel 8: Halten Sie die Sicherheitsvorschriften ein, die Sie vor einem Kontakt mit krebserregenden Stoffen am Arbeitsplatz schützen sollen.

Regel 9: Halten Sie die Belastung durch Radon zu Hause gering.

Regel 10: $\quad$ Für Frauen: Stillen schützt vor Brustkrebs. Stillen Sie daher Ihr Baby, wenn Sie können. Wenden Sie eine Hormonersatztherapie gegen Beschwerden in den Wechseljahren nur maßvoll an.

Regel 11: Lassen Sie Ihr Neugeborenes gegen Hepatitis-B und Mädchen gegen HPV impfen.

Regel 12: Gehen Sie regelmäßig zur Krebsfrüherkennungsuntersuchung.

(Modifiziert nach Association of European Cancer Leagues)

Bezogen auf die Ernährung werden darüber hinaus folgende Empfehlungen zur Krebsprävention gegeben: Vermehrter Verzehr pflanzlicher Lebensmittel wie Obst und Gemüse (fünf Portionen täglich) sowie ballaststoffreiche Vollkorn-Getreideprodukte, Reduktion der Zufuhr von Fett, rotem, verarbeitetem Fleisch und Salz sowie ein maßvoller Umgang mit Alkohol (Eichholzer, 2000; Eyre et al., 2004, Kushi et al., 2012). 


\section{Ernährungsverhalten und Ernährungsbildung}

\subsection{Obst- und Gemïseverzehr}

Obwohl vielen Menschen der gesundheitliche Nutzen einer obst- und gemüsereichen Ernährung bewusst ist, sieht der tatsächliche Verzehr häufig anders aus. Denn schon in den Erhebungen der Nationalen Verzehrsstudie I (NVS I), die zwischen 1985 und 1988 in den alten Bundesländern durchgeführt wurde, wird deutlich, dass zwar frischem Gemüse, Salat sowie frischem Obst die größte Bedeutung für eine gesunde Ernährung zugesprochen wird (Frauen $\approx 85 \% /$ Männer $\approx 75 \%$,sehr wichtig“); diese Vorstellungen aber nicht auf den Verzehr übertragen wurden (Kübler et al., 1994). In der Studie nahmen Männer im Durchschnitt täglich 152,3 g und Frauen 149 g Gemüse und Gemüseprodukte zu sich (Kübler et al., 1994). Bei Obst und Obstprodukten verzehrten Männer nur 106,4 g pro Tag während Frauen 130,6 g konsumierten (Kübler et al., 1994). Aktuelle Daten der bundesweit repräsentativen Nationalen Verzehrsstudie II (NVS II) aus 2008 bestätigen einen nach wie vor unzureichenden Obst- und Gemüseverzehr. 222 g Gemüse und Gemüseprodukte aßen Männer im Schnitt täglich, Frauen 243 g. Die von der Deutschen Gesellschaft für Ernährung empfohlene Menge von $400 \mathrm{~g}$ am Tag erreichten 88,5 Prozent der Männer und 86,3 Prozent der Frauen nicht (Max RubnerInstitut, 2008). Besser sieht es bei den Obst und Obsterzeugnissen aus. Männer nahmen durchschnittlich $222 \mathrm{~g}$ pro Tag zu sich und Frauen $270 \mathrm{~g}$. Aber auch hier unterschritten 65 Prozent der Männer und 54 Prozent der Frauen die von der DGE empfohlene Menge von 250 g pro Tag (Max Rubner-Institut, 2008).

\subsection{Obst- und Gemüseverzehr bei Kindern}

Insbesondere im Kindesalter spielt eine gesunde und ausgewogene Ernährung eine bedeutende Rolle (siehe Kapitel 2.3). Um dies zu gewährleisten, sieht das Forschungsinstitut für Kinderernährung (FKE) in Dortmund, folgende Empfehlungen für die altersgemäßen Lebensmittelverzehrmengen vor (Tabelle 2). 
Tabelle 2: Empfohlene altersgemäße Lebensmittelverzehrmengen

\begin{tabular}{|l|c|c|c|}
\hline Empfohlene Lebensmittel & $\mathbf{4}$ bis $\mathbf{6}$ Jahre & $\mathbf{7}$ bis 9 Jahre & 10 bis 12 Jahre \\
\hline Gemüse (g/Tag) & 200 & 220 & 250 \\
\hline Obst (g/Tag) & 200 & 220 & 250 \\
\hline Kartoffeln, Nudeln, Reis (g/Tag) & 180 & 220 & 270 \\
\hline Brot, Getreide (-flocken) (g/Tag) & 170 & 200 & 250 \\
\hline Fleisch, Wurst (g/Tag) & 40 & 50 & 60 \\
\hline Fisch (g/Woche) & 50 & 75 & 90 \\
\hline Eier (Stück/Woche) & 2 & 2 & $2-3$ \\
\hline Milch, -produkte (g/Tag) & 350 & 400 & 420 \\
\hline Öl, Margarine, Butter (g/Tag) & 25 & 30 & 35 \\
\hline Getränke (ml/Tag) & 800 & 900 & 1000 \\
\hline
\end{tabular}

Quelle: Modifizierte Darstellung nach Alexy et al., 2008, S. 171

Doch auch bei Kindern weicht das tatsächliche Ernährungsverhalten immer noch von den Empfehlungen deutlich ab, wie die bundesweit repräsentative Erhebung zur Gesundheit von Kindern und Jugendlichen in Deutschland, die vom Robert Koch-Institut zwischen 2003 und 2006 durchgeführt wurde, ergeben hat. Im Rahmen des Kinder- und Jugendgesundheitssurvey (KiGGS), an dem 17.641 Jungen und Mädchen im Alter von 0 bis 17 Jahren teilgenommen haben, wurde das Ernährungsverhalten mit Hilfe eines Verzehrhäufigkeitsfragebogens charakterisiert (Mensink et al., 2007a). In einer Nachfolgeerhebung des KiGGS, in der sogenannten „Ernährungsstudie als KiGGS-Modul“ (EsKiMo), wurde das Ernährungsverhalten von 2.506 Kindern und Jugendlichen im Alter von 6 bis unter 18 Jahren eingehender untersucht. In der Altersgruppe der 6- bis unter 12-Jährigen machten die Eltern in einem 3-TageVerzehrprotokoll (Ernährungstagebuch) Angaben zur Portionsgröße, Zubereitung, Produktbezeichnungen etc. der verzehrten Lebensmittel und Getränke (Deutsche Gesellschaft für Ernährung e.V., 2008).

Von besonderer Bedeutung für die Evaluation des Präventionsprojektes „5 am Tag für Kids“ sind die Ergebnisse zum Obst- und Gemüseverzehr bei den 6- bis 11-Jährigen. In dieser Altersgruppe lagen Daten aus 1.234 Ernährungstagebüchern mit insgesamt 3.677 Protokolltagen vor (Mensink et al., 2007b). Der Gemüseverzehr blieb weit unter den altersgemäßen Empfehlungen und wurde nur von 6 Prozent der Jungen und 7 Prozent der Mädchen erreicht. Beim Obstkonsum hingegen erreichten 15 Prozent der Jungen und 19 Prozent der Mädchen die empfohlenen Mengen (Mensink et al., 2007b). Demnach verzehrten Jungen im Alter von 7 bis 10 Jahren im Durchschnitt 106 g Gemüse und Gemüseprodukte pro Tag und $134 \mathrm{~g}$ Obst und Obstprodukte. Mädchen derselben Altersstufe aßen täglich durchschnittlich $107 \mathrm{~g}$ Gemüse und Gemüseprodukte sowie $142 \mathrm{~g}$ Obst und 
Obstprodukte (Deutsche Gesellschaft für Ernährung e.V., 2008). Ältere Daten aus der Einkommens- und Verbrauchsstichprobe 1998 belegen einen noch geringeren Verzehr. Demzufolge aßen Jungen zwischen 7- und 10 Jahren im Durchschnitt täglich nur 75 g Gemüse und 92 g Obst während Mädchen im Mittel 104 g Gemüse und 100 g Obst am Tag zu sich nahmen (Deutsche Gesellschaft für Ernährung e.V., 2004). Ähnliche Verzehrmengen ergaben sich auch in der ersten Nationalen Verzehrsstudie. Demnach lag die tägliche Aufnahme von Gemüse und Gemüseprodukten bei Jungen in der Altersgruppe 7 bis 9 Jahre bei 85,5 $\mathrm{g}$ und bei Obst und Obstprodukten bei 114,2 g; Mädchen aßen hingegen täglich im Durchschnitt 88,9 g Gemüse und 107,5 g Obst (Kübler et al., 1995).

Aus dem Verlauf der Datenerhebungen lässt sich ableiten, dass beim Verzehr von Obst und Gemüse in dieser Altersgruppe zwar ein leichter Anstieg zu verzeichnen ist, die empfohlenen Mengen jedoch trotz zunehmender Aktivitäten im Bereich „gesunde Ernährung“ nach wie vor nicht erreicht werden.

\subsection{Entwicklung des Ernährungsverhaltens}

Die Entwicklung von Ernährungsgewohnheiten, Geschmacksvorlieben und -abneigungen findet im Wesentlichen im frühen Kindesalter statt und bleibt auch im Erwachsenenalter relativ stabil (Patrick und Nicklas, 2005; Rasmussen, 2006). Eine Vielzahl von Faktoren spielt bei der Ausprägung der Verhaltensweisen eine Rolle: Sozio-ökonomische und kulturelle Faktoren, persönliche Aspekte, familienbezogene Faktoren sowie Einflüsse von peer-Group und Werbung (Patrick und Nicklas, 2005; Molderings, 2009).

$\mathrm{Zu}$ den persönlichen Faktoren zählen beispielsweise Geschmacksvorlieben. Die Vorliebe für süß ist angeboren und bestimmt von Beginn an die Auswahl von Lebensmitteln (Birch und Fisher, 1998; Ellrott, 2009; Scaglioni, 2011). Dagegen werden die Geschmacksrichtungen bitter und sauer abgelehnt (Birch und Fisher, 1998). Aufgrund des süßen Geschmacks ist Obst daher für Kinder auch attraktiver als Gemüse (Krølner et al., 2011). Darüber hinaus haben Kinder die Veranlagung, neue, unbekannte Lebensmittel abzulehnen und Verbindungen zwischen dem Geschmack und den postprandialen Auswirkungen nach dem Essen zu knüpfen (Birch und Fisher, 1998).

Die Familie spielt wohl die größte Rolle. Gerade in den ersten Jahren bestimmen die Eltern über die Verfügbarkeit und das Angebot von Lebensmitteln, über Mahlzeitenstruktur, Portionsgröße und Außer-Haus-Verzehr (Patrick und Nicklas, 2005; Ellrott, 2009). Ein weiterer 
wichtiger Aspekt bei der Entwicklung von Geschmacksvorlieben und Essgewohnheiten ist das Nachahmen von Vorbildern, das sogenannte Modelllernen (Patrick und Nicklas, 2005). Kinder orientieren sich an dem Verhalten ihrer Eltern oder anderer wichtiger Personen in ihrem Lebensumfeld (Birch und Fisher, 1998; Ellrott, 2009).

Dass auch sozioökonomische Aspekte wie das Einkommen und das Bildungsniveau der Eltern das kindliche Ernährungsverhalten beeinflussen, wurde in zahlreichen Studien belegt. Ein niedriges Haushaltseinkommen geht z.B. mit einem signifikant geringeren Einkauf von Obst und Gemüse einher (Molderings, 2009). Mit einem steigenden Bildungsniveau der Mutter, nimmt die Qualität der Ernährung zu (Molderings, 2009).

Mit zunehmendem Alter wirken sich Einflüsse von Werbung bzw. Fernsehen ebenfalls auf die Lebensmittelauswahl aus (Cullen et al., 2000; Blanchette und Brug, 2005). Oftmals werden hauptsächlich als nicht gesundheitsförderlich einzustufende Lebensmittel kommerziell beworben und nicht Obst und Gemüse (Krølner et al., 2011). Ein zunehmender Fernsehkonsum geht nachgewiesenermaßen mit einem verringerten Obst- und / oder Gemüseverzehr einher (Patrick und Nicklas, 2005; Rasmussen et al., 2006).

Anhand der nachfolgenden Abbildung 5 soll die Komplexität und das Zusammenwirken mannigfaltiger Faktoren, die zur Entwicklung des Ernährungsverhaltens beitragen und die die Lebensmittelauswahl beeinflussen, veranschaulicht werden: 
Abbildung 5: Influences on Food Choice

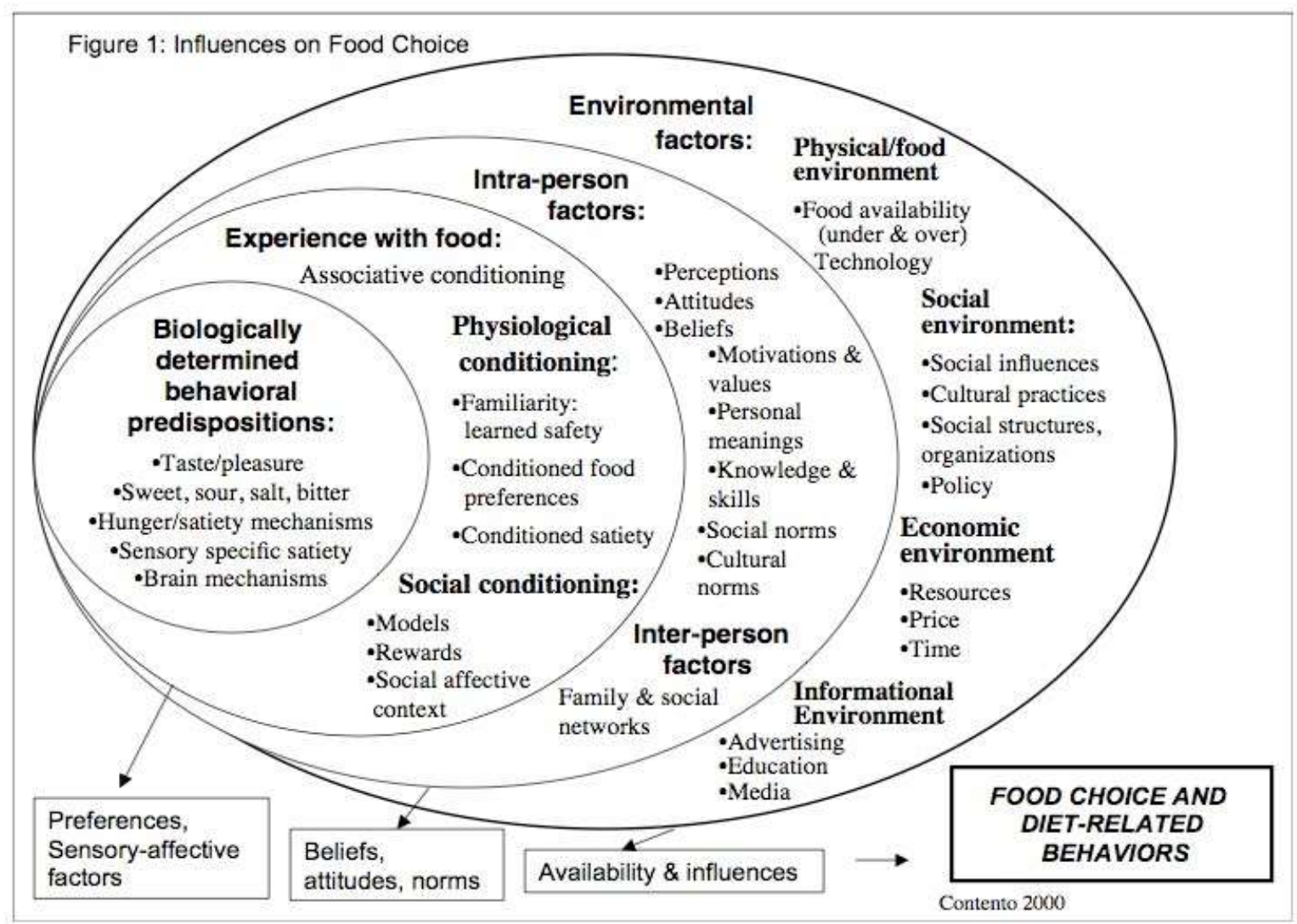

Quelle: Contento, 2008, S. 177

\subsection{Ernährungsbildung im Setting Schule}

Neben der Familie tragen sekundäre Sozialisationsinstanzen wie Kindertagesstätten und Schulen zur Prägung des Ernährungsverhaltens bei (Bundeszentrale für gesundheitliche Aufklärung, 2003; Bundeszentrale für gesundheitliche Aufklärung, 2009). Viele Familien sind heutzutage auch mit der Vermittlung von Wissen und Fähigkeiten im Umgang mit Essen überfordert, so dass Bildungseinrichtungen aufgefordert sind, diesen Defiziten durch eine zielgruppengerechte Ernährungsbildung entgegenzuwirken (Heindl, 2003; Heindl, 2009). Nachdem Forschungen jedoch offenbaren, dass insbesondere Menschen mit niedrigem Sozialund Bildungsstand von Verhaltensprävention in Form von kognitiven Aufklärungsstrategien und Expertenempfehlungen (traditionelle Ernährungserziehung) nicht erreicht werden, überträgt sich der Schwerpunkt auf institutionelle Programme in Erziehungs- und Bildungseinrichtungen im Bereich der Verhältnisprävention (Heindl, 2009). Das Ziel heutiger Ernährungsbildung ist daher der Kompetenzaufbau, „der die Lernenden zu reflektierten (möglichst gesundheitsförderlichen und nachhaltigen) Essentscheidungen in einer komplexen Welt befähigt“ (Bartsch, 2008). Der zunehmende Einfluss der Sozialisationsinstanzen auf die 
Ernährungsbildung der Kinder beruht auch auf der wachsenden Bedeutung des Pausenfrühstücks und dem Angebot der Mittagsverpflegung im Zuge des Ausbaus der Ganztagsbetreuung (Philipps, 2004). Die Institution Schule ist besonders geeignet, ernährungsbezogene Inhalte und Handlungsweisen systematisch zu vermitteln, da - bedingt durch die Schulpflicht - Kinder aus allen sozialen Schichten erreicht werden (Molderings, 2009). Darüber hinaus werden weitere relevante Zielgruppen wie Eltern, Erzieher und Lehrkräfte involviert (Heindl, 2009). Zudem verbringen die Schülerinnen und Schüler einen Großteil ihrer Zeit in der schulischen Umgebung und nehmen dort mindestens eine Mahlzeit ein (De Sa und Lock, 2007; Molderings, 2007). Auch unter Kosten-Nutzen-Aspekten werden Kindertagesstätten und Schulen als ideale Settings für Präventions- und Gesundheitsförderungsmaßnahmen angesehen (Heindl, 2009). Immer mehr gesundheitsfördernde Programme externer Institutionen wie „Klasse 2000“, „aidErnährungsführerschein“, Aktionen des Landfrauenverbandes o.ä. sind im Rahmen des Unterrichts angesiedelt, um den oben genannten Entwicklungen entgegen zu wirken.

Aber nicht nur externe Anbieter bringen gesundheitsförderliche Konzepte in die Schule, auch in den Lehrplänen sind inzwischen Themenfelder der Gesundheitsförderung verankert. So wurde ein Europäisches Kerncurriculum zur Ernährungsbildung mit sieben verschiedenen Themenfeldern entwickelt, das die strukturelle Voraussetzung für eine institutionelle Ernährungsbildung gründet. Hierdurch sollen den Kindern im Verlauf ihrer gesamten Schulzeit kontinuierlich vertiefende Ernährungsinhalte vermittelt werden (Heindl, 2009). Zu den Themenfeldern gehören:

- Essen und emotionale Entwicklung

- Essgewohnheiten, kulturelle und soziale Einflüsse

- Ernährung und persönliche Gesundheit

- Prozesse der Erzeugung, Verarbeitung und Verteilung von Lebensmitteln

- Lebensmittel, Märkte, Verbraucher und Konsum

- Konservierung und Lagerung von Lebensmitteln

- Kultur und Technik der Lebensmittelzubereitung

(Dixey et al., 2000; Heindl, 2009) 


\section{Die Gesundheitskampagne ,5-a-day“}

Der eingangs beschriebene Zusammenhang zwischen Ernährung, speziell dem Verzehr von Obst und Gemüse, und dem Auftreten von Krebs sowie weiteren chronischen Erkrankungen war der Grund, dass 1991 in Amerika das „5 A Day for Better Health Program“ initiiert wurde. Mit dieser Kampagne soll die Verzehrmenge von Obst und Gemüse bei der Bevölkerung gesteigert, und über die positiven gesundheitlichen Wirkungen dieser Lebensmittel aufgeklärt werden. Das Programm basiert auf vier Bausteinen: Massenmedien, Verkaufsstellen, kommunale Vereinigungen und Forschung.

Der Erfolg der Initiative wurde im Jahr 1999 evaluiert. Hierbei wurde die Teilnahme der Industrie, die Durchführung von Interventionen in der Gemeinde sowie die Berichterstattung in den Medien erfasst. Mit einer Prozessevaluation sollte festgestellt werden, ob die Kampagne auf die Zielgruppe zugeschnitten ist und die eingesetzten Materialien und Kommunikationsstrategien angemessen sind. Die Ergebnisevaluation lieferte Erkenntnisse, ob eine Änderung des Bewusstseins und des Wissens in der Bevölkerung stattgefunden hat. Anhand von zwei Surveys (baseline in 1991 und follow-up in 1997) wurden auch die Entwicklungen bezüglich des Obst- und Gemüseverzehrs beobachtet (Potter et al., 2000).

Von Interesse sind an dieser Stelle die Ergebnisse der Prozessevaluation. Während im Jahr 1991 nur zwei Prozent der Bevölkerung die Kampagne wahrgenommen hat, waren dies im Jahr 1997 immerhin 18 Prozent der Amerikaner. Die Bekanntheit war größer bei Frauen, jüngeren Personen, Weißen und Menschen mit höherer Bildung. Kenntnisse über die propagierten Verzehrempfehlungen (fünf oder mehr Portionen Obst und Gemüse am Tag) hatten beim ersten Survey nur acht Prozent der Bevölkerung, beim zweiten Survey immerhin 19 Prozent. Der mittlere Obst- und Gemüseverzehr stieg in den Jahren um 0,12 Portionen an (statistisch nicht signifikant), wobei diese Zunahme nicht zweifelsfrei auf die Maßnahmen des 5 A Day Program zurückzuführen sind (Potter et al., 2000).

http://www.fruitsandveggiesmorematters.org/ 


\section{1. „5 am Tag“6 in Deutschland}

Im Mai 2000 wurde in Deutschland der Verein 5 am Tag e.V. unter der Schirmherrschaft des Bundesministeriums für Gesundheit und des Bundesministeriums für Ernährung und Landwirtschaft gegründet, der den Verzehr von fünf Portionen Obst und Gemüse (etwa $650 \mathrm{~g}$ pro Tag) propagiert. Hinter dem Verein steht ein Netzwerk aus mehr als 100 Mitgliedern aus den Bereichen Gesundheit, Wirtschaft und Wissenschaft z.B. Fachgesellschaften, Krankenkassen, Ministerien oder Stiftungen. Durch eine enge Zusammenarbeit mit Unternehmen aus der Ernährungsindustrie, dem Lebensmittelhandel, der Obst- und Gemüseerzeugung sowie der Gemeinschaftsverpflegung und Gastronomie soll sichergestellt werden, dass die ,5-am-Tag“-Botschaft die Verbraucher vor Ort erreicht. Vielfältige Aktivitäten und Aktionen machen auf die Kampagne aufmerksam. Ein Expertengremium stellt die wissenschaftliche Begründung und Richtigkeit der Aussagen sicher. Zahlreiche weitere Länder beteiligen sich an der Aktion und propagieren die Botschaft länderspezifisch. Seit 2002 wird die Initiative von der Europäischen Union gefördert.

http://www.5amtag.org

\subsection{Projekt „5 am Tag für Kids - Spielend leicht vorbeugen mit Obst \& Gemüse“}

Basierend auf der Gesundheitskampagne „5 am Tag“ wurde im Jahr 2001 von „food \& future“ (Agentur für Kommunikation GmbH, Eichstr. 22, 67098 Bad Dürkheim, heute: food \& future - Agentur für Kommunikation \& Vertrieb e.K., Schumannstr. 12, 67269 Grünstadt) in Zusammenarbeit mit der Deutschen Krebsgesellschaft e.V. das Konzept sowie diverse Materialien für das fächerübergreifende Schulprojekt „5 am Tag für Kids“ entwickelt. Folgende Gründe sprachen für die Zielgruppe Kinder:

- Obst \& Gemüse sind „out“, Fast Food und Snacks sind „in“

- Kinder und Eltern sind besonders empfänglich für ,gesundheitsorientiertes Marketing“

- Wenn die Kinder begeistert sind, sind es die Eltern auch

- Die Ernährungsgewohnheiten werden im Kindesalter geprägt

- Kinder tragen die Botschaft der „5 am Tag“-Kampagne an andere Zielgruppen weiter

- Erreichbarkeit vieler Multiplikatoren: Eltern, Lehrkräfte, Journalisten, Sportvereine, Kinderärzte, Gemeinschaftsverpflegung

(food \& future, 2001) 
Im Jahr 2002 übernahm die Agentur „Das Trio“ (Das Trio - Kommunikation und Marketing GmbH, Käfertaler Str. 190, 68167 Mannheim) die PR und Kommunikationsarbeit für den Verein 5 am Tag e.V. und damit auch für das Schulprojekt.

Das entwickelte Konzept stand den 16 Länderkrebsgesellschaften zur Verfügung, die daraufhin die Umsetzung in den jeweiligen Bundesländern übernahmen. Folgende Länder haben das Projekt durchgeführt oder führen es bis heute durch:

- $\quad$ Baden-Württemberg

- Bayern

- Berlin (bis heute)

- Brandenburg (bis heute)

- Bremen (bis heute)

- Hamburg (bis heute)

- Hessen

- Niedersachsen

- Rheinland-Pfalz

- Saarland

- Schleswig-Holstein

- Thüringen

\section{Projektinhalte}

Zielgruppe für die Durchführung waren interessierte Grundschulen mit ihren 3. und 4. Klassen. In drei Unterrichtseinheiten sollten die Kinder dazu ermuntert werden, mehr Obst und Gemüse zu essen. In der schulischen Theorie-Einheit wurden die Portionsgrößen von Obst, Gemüse und Saft erläutert. Eine Portion entspricht einer Hand voll. Zudem lernten die Schülerinnen und Schüler die Farb-, Geruchs-, Geschmacks- und Abwehrstoffe der Pflanzen als Sekundäre Pflanzenstoffe anhand praktischer Beispiele kennen. Die positiven Wirkungen der Vitamine für den Körper wurden erklärt. Auch über den Einfluss der Ballaststoffe auf die Verdauung sowie die Aufnahme kanzerogener Substanzen wurden die Kinder informiert. In der WarenkundeEinheit, die jeweils in einem nahe gelegenen Lebensmittelmarkt stattfand, wurden verschiedene Obst- und Gemüsesorten betrachtet, verglichen und gewogen. Darüber hinaus wurden Informationen zur Saftkennzeichnung wie die Angaben zum Zuckergehalt und die Unterschiede im Fruchtgehalt von Fruchtsaft, -nektar und -saftgetränk vermittelt. Zum Schluss erhielt jedes Kind eine kleine Jutetasche mit Informationsmaterial und eine heimische Frucht 
als Wegzehrung. In der Schule wurde in der Praxis-Einheit das Gericht „Schatzinsel“ zubereitet. Die Kinder lernten den Umgang mit Küchenmesser und Sparschäler und richteten aus Kräuterquark, Mohrrüben, Paprika und Tomaten leckere und phantasievolle Teller her. Nebenbei erfuhren sie eine lebendige Esskultur. Mit Hilfe der Ernährungspyramide in Form eines riesigen Puzzles wurde den Kindern die ausgewogene und abwechslungsreiche Lebensmittelauswahl gezeigt. Abschließend wurden die teilnehmenden Schülerinnen und Schüler mittels Urkunde als ,5 am Tag“ - Kids ausgezeichnet.

Die erste Einheit vermittelt den Kindern das kognitive Hintergrundwissen über den gesundheitlichen Nutzen von Obst und Gemüse. Die zweite verhaltensorientierte Einheit soll sie dazu befähigen, dieses Wissen in eine geeignete Lebensmittelauswahl im Alltag zu übertragen. In der letzten praktischen Einheit sollen die Schülerinnen und Schüler ihre Kompetenzen hinsichtlich der Lebensmittelzubereitung trainieren und aufgrund von ModelLernen und peer education Freude am Probieren neuer Lebensmittel und der Zubereitung kleiner Gerichte gewinnen.

Folgende Materialien wurden im Rahmen des Projektes eingesetzt:

- Infomappen für interessierte Schulen

- Rezeptblätter „Schatzinsel“

- Urkunden

- Broschüre „5 am Tag - Obst \& Gemüse“, 5 am Tag e.V. und c/o Deutsche Krebsgesellschaft e.V. (Hrsg.) heute: „5 am Tag - Gesund mit Obst und Gemüse“, aid infodienst Verbraucherschutz, Ernährung, Landwirtschaft e.V. und 5 am Tag e.V. (Hrsg.)

- Elternbrief

- Kinderfaltblatt

- Diverse Infomaterialien des Vereins 5 am Tag e.V.

Die Niedersächsische Krebsgesellschaft e.V. hat das Projekt „5 am Tag für Kids“ im Zeitraum von August 2002 bis Dezember 2012 angeboten. In diesem Zeitraum fanden insgesamt 945 Schulprojekte mit knapp 20.000 Schülerinnen und Schülern statt. 395 Grundschulen aus allen 49 niedersächsischen Landkreisen beteiligten sich an der Aktion. Im November 2009 beschloss der Vorstand der Niedersächsischen Krebsgesellschaft, das Konzept wissenschaftlich zu evaluieren, um sowohl die Akzeptanz als auch den Erfolg der Intervention zu messen. 


\section{Literatur}

Alexy U., Clausen K. und Kersting M. 2008. Die Ernährung gesunder Kinder und Jugendlicher nach dem Konzept der Optimierten Mischkost. Ernährungs-Umschau, 03/08, 168-177.

Association of European Cancer Leagues. European Code Against Cancer (fourth version).

URL: http://cancer-code-europe.iarc.fr/images/doc/ecac_en.pdf (Zugriff am 25.06.2015).

Bartsch S. 2008. Subjektorientierung in der Ernährungs- und Gesundheitsbildung. Bundesgesundheitsblatt - Gesundheitsforschung - Gesundheitsschutz (3), 100-106.

Bayrisches Landesamt für Gesundheit und Lebensmittelsicherheit (Hrsg.) 2010. Evaluation in der Gesundheitsförderung. Eine Schritt-für-Schritt Anleitung für Gesundheitsförderer. Band 3 der Schriftenreihe Materialien zur Gesundheitsförderung, Erlangen.

Béliveau R. und Gingras D. 2007. Role of nutrition in preventing cancer. Canadian Family Physician. Vol. 53, 1905-1911.

Béliveau R. und Gingras D. 2008. Krebszellen mögen keine Himbeeren. Nahrungsmittel gegen Krebs. Das Immunsystem stärken und gezielt vorbeugen. Kösel-Verlag, München.

Benetou V., Orfanos P., Lagiou P., Trichopoulos D., Boffetta P. und Trichopoulou A. 2008. Vegetables and Fruits in Relation to Cancer Risk: Evidence from the Greek EPIC Cohort Study. Cancer Epidemiology, Biomarkers \& Prevention. 17 (2), 387-392.

Birch LL. und Fisher JO. 1998. Development of Eating Behaviors Among Children and Adolescents. Pediatrics. 101, 539-549.

Blanchette L. und Brug J. 2005. Determinants of fruit and vegetable consumption among 612-year-old children and effective interventions to increase consumption. Journal of Human nutrition and Dietetics, Vol. 18 (6), 431-443.

Boeing H., Bechthold A., Bub A., Ellinger S., Haller D., Kroke A., Leschik-Bonnet E., Müller MJ., Oberritter H., Schulze M., Stehle P. und Watzl B. 2012a. Critical review: vegetables and fruit in the prevention of chronic diseases. European Journal of Nutrition, Vol. 51, 637-663. 
Boeing H., Bechthold A., Bub A., Ellinger S., Haller D., Kroke A., Leschik-Bonnet E., Müller MJ., Oberritter H., Schulze M., Stehle P. und Watzl B. 2012b. Gemüse und Obst in der Prävention ausgewählter chronischer Krankheiten. Deutsche Gesellschaft für Ernährung e.V. (Hrsg.), Bonn, URL: http://www.dge.de/pdf/ws/DGE-Stellungnahme-GemueseObst-2012.pdf (Zugriff am 08.11.2012).

Boffetta P. et al. 2010. Fruit and vegetable intake and overall cancer risk in the European

Prospective Investigation into Cancer and Nutrition (EPIC). Journal of the National Cancer Institute, Vol. 102, 529-537.

Bradbury KE., Appleby PN. und Key TJ. 2014. Fruit, vegetable, and fiber intake in relation to cancer risk: findings from the European Prospective Investigation into Cancer and Nutrition (EPIC). American Journal of Clinical Nutrition, 100, 394S-398S.

Bundeszentrale für gesundheitliche Aufklärung (Hrsg.) 2003. Das Ernährungsverhalten Jugendlicher im Kontext ihrer Lebensstile. Band 20, Köln.

Bundeszentrale für gesundheitliche Aufklärung (Hrsg.) 2009. Gesundheitsförderung Konkret.

Unterrichtsmaterialien zur Ernährungserziehung in der Grundschule - eine bundesweite Bestandsaufnahme. Band 11, Köln.

Contento IR. 2008. Nutrition education: linking research, theory, and practice. Asia Pacific Journal of Clinical Nutrition.

Cullen KW., Baranowski T., Rittenberry L. und Olvera N. 2000. Social-environmental influences on children's diets: results from focus groups with African-, Euro- and Mexican-American children and their parents. Health Education Research. Vol. 15, no. $5,581-590$.

Dauchet L., Amouyel P., Hercberg S. und Dallongeville J. 2006. Fruit and vegetable consumption and risk of coronary heart disease: A meta-analysis of cohort studies. The Journal of Nutrition. Vol. 136, no. 10, 2588-2593.

De Sa J. und Lock K. 2007. School-based fruit and vegetable schemes: A review of the evidence. London School of Hygiene and Tropical Medicine. URL: http://www.5aday.co.nz/5adaywebsite/cms_resources/file/SCHOOL\%20REVIEW\%2 02007.pdf (Zugriff am 12.05.2011).

Deutsche Adipositas-Gesellschaft (DAG) e.V., Deutsche Diabetes Gesellschaft (DDG), Deutsche Gesellschaft für Ernährung (DGE) e.V., Deutsche Gesellschaft für Ernährungsmedizin (DGEM) e.V. (Hrsg.) 2014. Interdisziplinäre Leitlinie der Qualität S3 zur „Prävention und Therapie der Adipositas“. Version 2.0. 
Deutsche Gesellschaft für Ernährung e.V. (Hrsg.) 2004. Ernährungsbericht 2004. Bonn.

Deutsche Gesellschaft für Ernährung e.V. (Hrsg.) 2007. Obst und Gemüse in der Prävention chronischer Krankheiten.

Deutsche Gesellschaft für Ernährung e.V. (Hrsg.) 2008. Ernährungsbericht 2008. Bonn.

Deutsche Gesellschaft für Ernährung e.V. (Hrsg.) 2012. Obst und Gemüse in der Prävention chronischer Krankheiten.

Deutsche Gesellschaft für Ernährung e.V., Österreichische Gesellschaft für Ernährung, Schweizerische Gesellschaft für Ernährungsforschung, Schweizerische Vereinigung für Ernährung (Hrsg.) 2015. Referenzwerte für die Nährstoffzufuhr. Bonn, 2. Auflage, 1. Ausgabe.

Dixey R., Heindl I., Loureiro I., Pérez-Rodrigo C., Snel J. und Warnking P. 2000. Healthy eating for young people in Europe - a school-based nutrition education guide. WHO. URL: http://www.euro.who.int/_data/assets/pdf_file/0005/119921/E69846.pdf (Zugriff am 03.09.2014).

Doll R. und Peto R. 1981. The causes of cancer: quantitative estimates of avoidable risks of cancer in the United States today. Journal of the National Cancer Institute. Vol. 66, no. 6, 1192-1308.

Donaldson MS. 2004. Nutrition and cancer: A review oft he evidence for an anti-cancer diet. Nutrition Journal. 3:19.

Dorai T. und Aggarwal BB. 2004. Role of chemopreventive agents in cancer therapy. Cancer Letters. 215, 129-140.

Eichholzer M. 2000. Ernährung und Krebs. In: Therapeutische Umschau. Band 57, Heft 3, Verlag Hans Huber, Bern, 146-151.

Eyre H., Kahn R. und Robertson RM. 2004. Preventing Cancer, Cardiovascular Disease, and Diabetes. A common agenda for the American Cancer Society, the American Diabetes Association, and the American Heart Association. Diabetes Care. Vol. 27, no. 7, 18121824.

Food \& future - Agentur für Kommunikation GmbH 2001. Profilierung der DKG als innovativer und führender Gesundheitspartner der "5 am Tag"-Kampagne. Präsentation. 
Gerhäuser C. 2011. Schach dem Krebs? Einfluss sekundärer Pflanzenstoffe auf die

Karzinogenese - Keeping Cancer in Check? The Influence of Phytochemicals on Carcinogenesis. Aktuelle Ernährungsmedizin. 36 (1), 518-522.

Gescher A., Pastorino U., Plummer SM. und Manson MM. 1998. Suppression of tumour

development by substances derived from the diet: mechanism and clinical implications.

British Journal of Clinical Pharmacology. 45, 1-12.

Gonzalez CA. und Riboli E. 2010. Diet and cancer prevention: Contributions from the European

Propective Investigation into Cancer and Nutrition (EPIC) study. European Journal of Cancer. 46, 2555-2562.

Heindl I. 2003. Studienbuch Ernährungsbildung - Ein europäisches Konzept zur schulischen

Gesundheitsförderung. Verlag Julius Klinkhardt, Bad Heilbrunn.

Heindl I. 2009. Ernährungsbildung - curriculare Entwicklung und institutionelle

Verantwortung. Ernährungs Umschau. 56, 568-573.

Horne PJ., Tapper K., Lowe CF., Hardman CA., Jackson MC. Und Woolner J. 2004.

Increasing children's fruit and vegetable consumption: a peer modeling and rewardsbased intervention. European Journal of Clinical Nutrition, 58, 1649-1660.

Howerton MW., Bell BS., Dodd KW., Berrigan D., Stolzenberg-Solomon R. und Nebeling L. 2007. School-based Nutrition Programs Produced a Moderate Increase in Fruit and Vegetable Consumption: Meta and Pooling Analyses from 7 Studies. Journal of Nutrition Education and Behavior, 39, 186-196.

Hurrelmann K., Klotz T. und Haisch J. (Hrsg.). 2010. Lehrbuch Prävention und

Gesundheitsförderung. 3. Vollständig überarbeitete und erweiterte Auflage, Verlag Hans Huber, Hogrefe AG, Bern.

Hyson D. 2002. The Health Benefits of Fruits and Vegetables. A Scientific Overview for Health Professionals. Wilmington, DE: Produce for Better Health Foundation. URL: http://www.polymva.com/pdf/Fruits-and-Vegetables.pdf (Zugriff am 04.08.2011).

Key TJ., Schatzkin A., Willet WC., Allen NE., Spencer EA. Und Travis RC. Diet, nutrition and the prevention of cancer. Public Health Nutrition. 7, 187-200.

Key TJ. 2011. Minireview: Fruit and vegetables and cancer risk. British Journal of Cancer. 104, 6-11. 
Kipping RR., Howe LD., Jago R., Campbell R., Wells S., Chittleborough CR., Mytton J., Noble SM., Peters TJ. und Lawlor DA. Effect of intervention aimed at increasing physical activity, reducing sedentary behaviour, and increasing fruit and vegetable consumption in children: Active for Life Year 5 (AFLY5) school based cluster randomised controlled trial. British Medical Journal, 348:g3256.

Klepp KI., Pereze-Rodrigo C., De Bourdeaudhuij I., Due P., Elmadfa I., Haraldsdottir J., Konug J., Sjostrom M., Thorsdottir I., Vaz de Almeida MD., Yngve A. und Brug J. 2005. Promoting Fruit and Vegetable Consumption among European Schoolchildren: Rationale, Conceptualization and Design of the Pro Children Project. Annals of Nutrion \& Metabolism. 49, 212-220.

Klotz T., Haisch J. und Hurrelmann K. 2006. Prävention und Gesundheitsförderung. Ziel ist anhaltend hohe Lebensqualität. Deutsches Ärzteblatt. 103(10), A606-A609.

Knasmüller S., Mišík M., Parzefall W. und Wagner KH. (2014) Krebs und Ernährung - Risiken und Prävention - wissenschaftliche Grundlagen und Ernährungsempfehlungen, Georg Thieme Verlag KG, Stuttgart.

Krølner R., Rasmussen M., Brug J., Klepp KI., Wind M. und Due P. (2011) Determinants of fruit and vegetable consumption among children and adolescents: a review of the literature. Part II: qualitative studies. International Journal of Behavioral Nutrition and Physical Activity. 8:112.

Kushi LH., Byers T., Doyle C., Bandera EV., McCullough M., Gansler T., Andrews KS., Thun MJ. and The American Cancer Society 2006 Nutrition and Physical Activity Guidelines Advisory Committee. 2006. American Cancer Society Guidelines on Nutrition and Physical Activity for Cancer Prevention: Reducing the Risk of Cancer With Healthy Food Choices and Physical Activity. CA: A Cancer Journal for Clinicians. 56, 254-281.

Kushi LH., Doyle C., McCullough M., Rock CL., Demark-Wahnefried W., Bandera EV., Gapstur S., Patel AV., Andrews K., Gansler T. and The American Cancer Society 2010 Nutrition and Physical Activity Guidelines Advisory Committee. 2012. American Cancer Society Guidelines on Nutrition and Physical Activity for Cancer Prevention: Reducing the Risk of Cancer With Healthy Food Choices and Physical Activity. CA: A Cancer Journal for Clinicians. 62, 30-67. 
Kübler W., Anders HJ., Heeschen W. und Kohlmeier M. (Hrsg.). 1994. Lebensmittel- und Nährstoffaufnahme Erwachsener in der Bundesrepublik Deutschland. VERASchriftenreihe, Band III, Zweite, überarbeitete Auflage, Wissenschaftlicher Fachverlag Dr. Fleck, Niederkleen.

Kübler W., Anders HJ. und Heeschen W. (Hrsg.). 1995. Ergebnisse der Nationalen

Verzehrsstudie (1985-1988) über die Lebensmittel- und Nährstoffaufnahme in der Bundesrepublik Deutschland. VERA-Schriftenreihe, Band XI, Wissenschaftlicher Fachverlag Dr. Fleck, Niederkleen.

Liu RH. 2004. Potential Synergy of Phytochemicals in Cancer Prevention: Mechanism of Action. The Journal of Nutrition. Vol. 134, no. 12, 3479S-3485S.

Lowe CF., Horne PJ., Tapper K., Bowdery M. und Egerton C. 2004. Effects of a peermodelling and rewards-based intervention to increase fruit and vegetable consumption in children. European Journal of Clinical Nutrition, 58, 510-522.

Luengo-Fernandez R., Leal J., Gray A. und Sullivan R. 2013. Economic burden of cancer across the European Union: a population-based cost analysis. The Lancet Oncology, Vol. 14, 1165-1174.

Max Rubner-Institut - Bundesforschungsinstitut für Ernährung und Lebensmittel (Hrsg.). 2008. Nationale Verzehrsstudie II - Die Bundesweite Befragung zur Ernährung von Jugendlichen und Erwachsenen. Ergebnisbericht, Teil 2. Karlsruhe.

Medizinischer Dienst des Spitzenverbandes Bund der Krankenkassen e.V. (MDS) und GKVSpitzenverband (Hrsg.). 2014. Präventionsbericht 2014. Leistungen der gesetzlichen Krankenversicherung: Primärprävention und betriebliche Gesundheitsförderung. Berichtsjahr 2013.

Mensink GBM. und Burger M. 2004. Was isst du? Ein Verzehrshäufigkeitsfragebogen für Kinder und Jugendliche. Bundesgesundheitsblatt - Gesundheitsforschung Gesundheitsschutz, Springer-Verlag, 47, 219-226.

Mensink GBM., Kleiser C. und Richter A. 2007a. Lebensmittelverzehr bei Kindern und Jugendlichen in Deutschland. Ergebnisse des Kinder- und Jugendgesundheitssurveys (KiGGS). Bundesgesundheitsblatt - Gesundheitsforschung - Gesundheitsschutz, Springer Medizin Verlag, 50, 609-623.

Mensink GBM., Heseker H., Richter A., Stahl A. und Vohmann C. 2007b. Forschungsbericht Ernährungsstudie als KiGGS-Modul (EsKiMo). 
Molderings M. 2007. Evaluation pädagogisch-didaktischer Ansätze im Rahmen der gesundheitsförderlichen Ernährungserziehung der Grundschule: Einfluss von Unterrichtsformen, Elterneinbeziehung und Zielvereinbarung hinsichtlich einer Veränderung des Ernährungsverhaltens. Schneider Verlag Hohengehren [Dissertation], Baltmannsweiler.

Molderings M. 2009. Wie Kinder ihre Eltern erziehen - Kann Schule die Ernährung zu Hause verändern? In: Ritterbach U., Nicolaus J., Spörhase U., Schleider K. (Hrsg). 2009. Leben nach Herzenslust? Lebensstil und Gesundheit aus psychologischer und pädagogischer Sicht. Freiburg im Breisgau: Centaurus (Schriftenreihe der Pädagogischen Hochschule Freiburg; 20), 83-103.

Österreichische Akademie für Arbeitsmedizin und Prävention. Primär-, Sekundär- und Tertiärprävention. URL: http://www.aamp.at/unsere-themen/praevention/primaersekundaer-und-tertiaerpraevention/ (Zugriff am 07.08.2014).

Patrick H. und Nicklas TA. 2005. A Review of Family and Social Determinants of Children's Eating Patterns and Diet Quality. Journal of the American College of Nutrition. Vol. 24, no. 2, 83-92.

Philipps U. 2004. Evaluation gesundheitsfördernder Maßnahmen bezüglich des

Ernährungsverhaltens von Grundschulkindern. Verlag Julius Klinkhardt, [Dissertation], Bad Heilbrunn.

Potter JD., Finnegan JR., Guinard JX., Huerta EE., Kelder SH., Kristal AR., Kumanyika S., Lin R., McAdams Motsinger B., Prendergast FG., Sorensen G. und Callahan KM. 2000. 5 A Day for Better Health Program Evaluation Report. National Institutes of Health und National Cancer Institute (Hrsg.). November 2000. NIH Publication No. 01-4904.

Rasmussen M., Krølner R., Klepp KI., Lytle L., Brug J., Bere E. und Due P. 2006.

Determinants of fruit and vegetable consumption among children and adolescents: a review of the literature. Part I: quantitative studies. International Journal of Behavioral Nutrition and Physical Activity. 3:22.

Registerstelle des Epidemiologischen Krebsregisters in Niedersachsen (EKN) (Hrsg.) 2014. Krebs in Niedersachsen 2011. Oldenburg.

Riboli E. und Norat T. 2003. Epidemiologic evidence of the protective effect of fruit and vegetables on cancer risk. The American Journal of Clinical Nutrition, 78, 559S-569S.

Robert Koch-Institut und Die Gesellschaft der epidemiologischen Krebsregister in Deutschland e.V. (Hrsg.) 2013. Krebs in Deutschland 2009/2010. 9. Ausgabe, Berlin. 
Sachverständigenrat für die Konzertierte Aktion im Gesundheitswesen (Hrsg.) 2000/2001.

Bedarfsgerechtigkeit und Wirtschaftlichkeit. Band I - Zielbildung, Prävention, Nutzerorientierung und Partizipation. Band II - Qualitätsentwicklung in Medizin und Pflege. Gutachten.

Scaglioni S., Arrizza C., Vecchi F. und Tedeschi S. 2011. Determinants of children's eating behavior. American Journal of Clinical Nutrition. 94, 2006S-2011S.

Slavin JL. und Lloyd B. 2012. Health Benefits of Fruits and Vegetables. American Society for Nutrition. Advances in Nutrition, 3, 506-516.

Slesina W. 2007. Primordiale, orimäre, sekundäre und tertiäre Prävention - Eine Begriffsbestimmung. Deutsche Medizinische Wochenschrift. 132, 2196-2198.

Statistisches Bundesamt 2014. Krankheitskosten Deutschland nach Krankheitsdiagnosen (ICD10). URL: https://www.genesis.destatis.de/genesis/online;jsessionid=52053892E 0480C7A868F1A83E334CA95.tomcat_GO_1_2?operation=previous\&levelindex=2\& levelid=1405414399215\&step=2 (Zugriff am 15.07.2014, Stand 10.53 Uhr).

Statistisches Bundesamt 2014. Todesursachen. URL: https://www.destatis.de/DE/ZahlenFakt en/GesellschaftStaat/Gesundheit/Todesursachen/Tabellen/GestorbeneAnzahl.html (Zugriff am 15.07.2014).

Van Cauwenberghe E., Maes L., Spittaels H., van Lenthe FJ., Brug J., Oppert JM. und De Bourdeaudhuij I. 2010. Effectiveness of school-based interventions in Europe to promote healthy nutrition in children and adolescents: systematic review of published and 'grey' literature. British Journal of Nutrition. 103, 781-797.

Van Duijnhoven FJB. Et al. 2009. Fruit, vegetables, and colorectal cancer risk: the European Prospective Investigation into Cancer and Nutrition. American Journal of Clinical Nutrition. 89, 1441-1452.

Willich SN. 2007. Prävention - Gesundheitliche Wertschöpfung. Deutsches Ärzteblatt. Jg. 104, Heft 26, A 1893-A 1895.

Wiseman M. 2008. The Second World Cancer Research Fund/American Institute for Cancer Research Expert Report. Food, Nutrition, Physical Activity, and the Prevention of Cancer: A Global Perspektive. In: Nutrition Society and BAPEN Medical Symposium on "Nutrition support in cancer therapy". Proceedings of the Nutrition Society. 253-256.

World Cancer Research Fund and American Association for Cancer Research (Hrsg.). 1997.

Diet and the Cancer Process in: Food, Nutrition, Physical Activity and the Prevention of Cancer: a Global Perspective. American Institute for Cancer Research, Washington, DC. 
World Cancer Research Fund and American Institute for Cancer Research (Hrsg.). 2007.

Zusammenfassung - Ernährung, körperliche Aktivität und Krebsprävention: Eine globale Perspektive. 1st ed, World Cancer Research Fund International, London.

World Health Organization (Hrsg.). 2003. Diet, Nutrition and the Prevention of Chronic

Diseases: Report of a Joint WHO/FAO Expert Consultation. Geneva. 28 January-1 February 2002. WHO Technical Report Series 916. 


\section{Eigene Beiträge}

Zahlreiche „5 a day“ Interventionen wurden bereits wissenschaftlich auf ihre Wirksamkeit hin untersucht, allerdings vorwiegend in Amerika oder anderen europäischen Ländern. Dabei zeigen Studien, dass Interventionen, die mehrere Bausteine miteinander kombinieren, z.B. ein Curriculum für Ernährungsbildung, ein verbessertes Angebot von Obst und Gemüse in der Schule sowie der Einbezug der Eltern, erfolgsversprechender sind (Blanchette und Brug, 2005; Howerton et al., 2007; Van Cauwenberghe et al., 2010) als vorwiegend wissensbasierte Interventionen (Kipping et al., 2014).

Seit fast 15 Jahren wird in Deutschland das Projekt „,5 am Tag für Kids“ von mehreren Landeskrebsgesellschaften durchgeführt. Eine große Anzahl an Kindern wurde auf diesem Weg zum Thema Obst und Gemüse geschult. Bisher lagen keine Erkenntnisse bezüglich der Akzeptanz der Maßnahme und vor allem bezüglich der Effekte hinsichtlich der erwünschten Steigerung des Obst- und Gemüseverzehrs der teilnehmenden Dritt- und Viertklässler von Grundschulen vor. Aus diesem Grund initiierte die Niedersächsische Krebsgesellschaft die wissenschaftliche Begutachtung des Projektes. Darüber hinaus soll die vorliegende Dissertation dazu beitragen, die Konzeptionierung schulbasierter Interventionen $\mathrm{zu}$ verbessern und erfolgsversprechende Elemente herauszufiltern.

Der erste Beitrag „Behavioural Effects of a Short School-Based Fruit and Vegetable Promotion Programme: 5 a Day for Kids“ (siehe S. 39) thematisiert die Evaluation des Projektes „,5 am Tag für Kids“. Die Ergebnisse basieren auf einer Erhebung, die zwischen Januar 2011 und Januar 2012 an 69 niedersächsischen Grundschulen durchgeführt wurde. Die Stichprobe schloss 1.376 Schülerinnen und Schüler sowie deren Eltern und 69 Lehrkräfte ein. Die Ergebnisse zeigen, dass die Intervention zwar sowohl von den Kindern als auch von den Lehrkräften gut angenommen und bewertet wird, aber keine kurz- oder mittelfristigen Auswirkungen auf den Obst- und Gemüseverzehr der Schülerinnen und Schüler mit der gewählten Erhebungsmethode (Food Frequency Questionnaire) nachzuweisen sind. Aufgrund dieser Erkenntnisse, wird in diesem Beitrag auch über mögliche Maßnahmen zur Steigerung der Effektivität diskutiert.

Der zweite Beitrag mit dem Titel „Welche Faktoren sind mit dem Obst- und Gemüseverzehr von Grundschülern assoziiert?“ (siehe S. 62) greift den Themenkomplex auf, welche Einflussfaktoren auf das kindliche Essverhalten wirken. Diese Erkenntnisse sollen dazu dienen, 
sowohl das Projekt „5 am Tag für Kids“ als auch andere vorwiegend wissensbasierte Konzepte im Bereich der Ernährungsbildung zu verbessern und um Aspekte zu ergänzen, die sich positiv auf die Effektivität auswirken. Die Ergebnisse zeigen, dass das von den Eltern zur Verfügung gestellte Angebot von Obst und Gemüse sowohl in der häuslichen Umgebung als auch in der Schule, den kindlichen Verzehr dieser Lebensmittel maßgeblich beeinflusst. Damit bestätigt sich, dass die Implementierung eines landesweiten (kostenlosen) Schulfruchtprogramms einen sinnvollen und vielversprechenden Ansatz darstellt. Auf diesem Weg könnten Bildungseinrichtungen zumindest zum Teil häusliche Defizite ausgleichen.

Der dritte Beitrag „Praktische Ernährungsbildung in der 5./6. Klasse: Evaluation des Projekts 'Entdeckungsreise Essen'“ (siehe S. 74) fügt sich in den Kontext 'Vermittlung von Ernährungskompetenzen im Rahmen schulischer Ernährungsbildung' ein. Bei der „Entdeckungsreise Essen“ handelt es sich um ein modulbasiertes, gesundheitsförderliches Ernährungskonzept für die Sekundarstufe 1, das auf einem partizipativen, explorierenden und praktischen Ansatz basiert. Dieses Projekt wurde vom Institut für Ernährungspsychologie an der Universitätsmedizin Göttingen in Kooperation mit weiteren Praxispartnern entwickelt. Bei der Konzipierung wurde besonderer Wert auf die erlebnis-, schüler- und handlungsorientierte Ausrichtung der Intervention sowie auf den ressourcensparenden und flexiblen Einsatz der Module für die Schulen gelegt. Die Lehrkräfte sollen durch die einfache Integration der Module in den Regelunterricht ebenfalls miteinbezogen werden.

Einzelne ausgewählte Module wurden auf ihre Bewertung und Wirksamkeit hin untersucht. Erste Ergebnisse zeigen, dass die praktische, teilhabende Intervention von den Kindern sehr positiv angenommen wird. Je höher die Partizipation und die Praxisanteile eines Moduls ausfielen, desto höher war die Attraktivität für die Schüler. Zudem wurden im Projekt erlernte Verhaltensweisen und Handlungskompetenzen auch auf den Alltag zu Hause übertragen z.B. bereiteten die Kinder Rezepte zu Hause zu. 


\section{Behavioural Effects of a Short School-Based Fruit and Vegetable Promotion Programme: 5-a-Day for Kids}

Autoren: Silke Mittmann, Anja Austel, Thomas Ellrott

Erscheint in Health Education, 2016, Vol. 116 (3), Emerald Group Publishing, im Druck

This article is (C) Emerald Group Publishing and permission has been granted for this version to appear here (https://ediss.uni-goettingen.de/). Emerald does not grant permission for this article to be further copied/distributed or hosted elsewhere without the express permission from Emerald Group Publishing Limited. 


\begin{abstract}
Purpose - The purpose of this paper was to investigate the effects of the Cancer Society of Lower Saxony's, Germany's school-based nutrition education programme "5-a-day for kids", designed to increase children's fruit and vegetable consumption.

Intervention - The programme included three parts (each 45 minutes): 1. Education based classroom session, 2. Food knowledge in a local supermarket, 3. Practical vegetable snack preparation. Additional informational materials for parents were provided.
\end{abstract}

Design/methodology/approach - A pre-/post-test research design was used for the evaluation. 1,376 pupils (age 7-14, 51\% female), their parents and 69 teachers of 35 schools in Lower Saxony participated in the study. The fruit and vegetable intake was measured with the KiGGSFood Frequency Questionnaire.

Findings - A Wilcoxon signed-rank test was used to determine the change in fruit and vegetable consumption over three measurements (baseline, one month, three months). No significant positive effect of the intervention was observed with the applied method for the daily intake of fruit and vegetables, neither at month 1 nor at month 3 follow up.

Implications - A 135 min. school-based intervention does not seem to increase children's fruit and vegetable consumption. To enhance its effectiveness, the programme might be improved by adding a longitudinal classroom component, extensive parental involvement and/or distribution of free fruit/vegetables every day.

Originality/value - This is the first evaluation of a 5-a-day-intervention in Germany.

Key Words - fruit and vegetables, schools, intervention, child nutrition, 5-a-day 


\section{INTRODUCTION}

Research has indicated that eating a diet rich in fruit and vegetables has important health benefits. Higher fruit and vegetable intake has been associated with a lower risk of developing cancer, cardiovascular disease, hypertension, stroke, obesity and other diseases [World Health Organization, 2003; Hyson 2011; Boeing et al., 2012]. Because of these correlations, the U.S. National 5-A-Day for Better Health Program, initiated in 1991 by the National Cancer Institute and the Produce for Better Health Foundation, promotes the consumption of fruit and vegetables by Americans of five or more servings per day [Heimendinger and Van Duyn, 1995; Potter et al., 2000]. In the year 2000, the registered association "5-a-day" was founded in Germany and has also been supporting the increase of consuming these foods.

In accordance with the recommendations of the German Research Institute of Child Nutrition, children aged 7 to 9 are required to consume 220 gram of fruit and 220 gram of vegetables a day and children at the age of 10 to 12 should ingest 250 gram of both [Alexy et al., 2008]. However, German children do not eat the recommended amounts of fruit and vegetables: only $6 \%$ of boys and $7 \%$ of girls aged between 6 and 11 achieve these recommendations for vegetables and only $15 \%$ of the boys and $19 \%$ of the girls attain the recommended daily amount of fruit [Mensink et al., 2007a]. Girls at the age of 7 to 10 eat an average of 106g of vegetables and $141 \mathrm{~g}$ of fruit, while boys consume $105 \mathrm{~g}$ of vegetables and $132 \mathrm{~g}$ of fruit [Mensink et al., 2007a; Deutsche Gesellschaft für Ernährung e.V., 2008].

Even if parents are predominantly responsible for the availability of food, there is evidence that children influence what they eat. Food promotion already influences the food preferences and the purchase behaviour of primary schoolchildren. For example, children asked their mothers to buy products because of television's influence [Hastings et al., 2003]. Normally they get pocket money, so they can buy food or snacks in the school environment or in the supermarket themselves [Roberts et al., 2003]. Moreover, there are hints that schoolchildren strongly influence their lunchbox content [Roberts et al., 2003].

Previous reports have pointed out that a dietary intake of healthy foods should be established in childhood because this early learned behaviour often persists throughout a person's whole life [Burchett, 2003; Gerhards and Rössel, 2003; Bundeszentrale für gesundheitliche Aufklärung, 2009; Van Cauwenberghe et al., 2010] and may have considerable impact on the 
long-term health-status [Livingstone and Robson, 2000; World Health Organization, 2003]. That is why intervening in children's consumption may be more successful than influencing the ingrained eating habits of adults [Burchett, 2003]. School-based nutrition programmes have the potential to be a very effective way of changing fruit and vegetable intake because the children spend a lot of time in the school environment and usually consume at least one meal there [De Sa and Lock, 2007; Van Cauwenberghe et al., 2010]. A number of studies have previously observed the effects of "5-a-day" interventions. Reviews concluded that even multicomponent school-based interventions only had a moderate effect on increasing fruit and vegetable intake [Van Cauwenberghe et al., 2010; Howerton et al., 2007] or only a minimal impact on vegetable consumption [Evans et al., 2012]. However, there were still some components which showed significant effects in the following studies. For example, "Gimme 5" (Georgia, USA) - which is based on 12 classroom sessions each year (fourth and fifth grades), newsletters taken home to parents, videotapes and point-of-purchase education - obtained an effective increase of +0.2 servings in the 2000 study [Baranowki et al. 2000] and +0.3 servings in the original "Gimme 5" undertaken in 1993 [Domel et al., 1993]. Significantly more fruit and vegetables were consumed in the intervention group than in the control group, but this was due to a decline in consumption in the control group rather than an increase in consumption in the treatment group [Burchett, 2003]. The "5-a-Day Power Plus" intervention consisted of four components: behavioural curricula (16 classroom sessions in the fourth and fifth grades), parental involvement, school food service changes, and industry support. Intervention pupils had a higher mean intake of fruit and vegetable ( +0.4 servings/day) than the control group [Perry et al., 1998]. Single-component environmental programmes such as school fruit programmes which provide a free piece of fruit per day have also had significant short-and long-term effects on children's fruit and vegetable intake [Bere et al., 2006a; Bere et al., 2006b; Bere et al., 2007].

The aim of the current study was to evaluate the acceptance of the scheme as well as the shortand intermediate-term effects of the German "5-a-day for kids" project. This short school-based fruit and vegetable programme has been offering at no cost for interested primary schools by the Cancer Society of Lower Saxony, Germany.

\section{Intervention}

Based on the American health campaign "5-a-day", the advertising agency "food \& future" developed a marketing concept for an intervention in primary schools in cooperation with the German Cancer Society. The target group "schoolchildren" were chosen because they eat less 
fruit and vegetables than recommended. Eating behaviour is predominantly established in childhood; children and parents are amenable to health-oriented marketing, and furthermore, the setting school offers a platform to reach many multipliers like parents, teachers, journalists and staff in the supermarket. Nutrition education research suggest that parents can be reached through their children [Klepp et al., 2005]. The programme was designed to be fun and participative while targeting behaviour change. The focus was on "edutainment", that means to combine education and entertainment to enhance motivation for learning.

The Cancer Society of Lower Saxony implemented the project "5-a-day for Kids" in August 2002. Up to December 2012, more than 20,000 third- and fourth-grade pupils from 396 different schools in Lower Saxony have taken part in this prevention programme. In addition, at the beginning the programme was undertaken by nine further federal states of Germany.

The one-day intervention took about 135 Minutes and included three parts.

Part 1 was an educationally based classroom session in which the pupils learned about portion size and nutrients that are beneficial for health. Technical terms, for example vitamins, mineral nutrients, phytochemicals or fibre were explained in a child appropriated way.

Part 2 took place in a local supermarket. During this session, the children got to know the variety of fruit and vegetables and the differences between $100 \%$ fruit juice, juice drink and squash. At the end of this part, they got a bag within information leaflets relating to the campaign "5-a-day" and the health benefits of eating fruit and vegetables (the booklet "5 a day - healthy with fruit and vegetables", a letter for their parents, child-oriented information material to paint in) and generally one piece of fruit. For homework, the pupils had to go through this material together with their parents. In this way, the parents were invited to be involved and to get to know what their child had learned about fruit and vegetables.

In Part 3, the pupils prepared a healthy vegetable snack, tasted it and received information about preparing recipes at home. The task of preparing recipes at home was intended to involve the parents and to enhance the preparation skills of the children.

Part 1 provided the background knowledge about nutrients and the health benefits of fruit and vegetables to enable the children to make healthy food choices not only in the supermarket but 
also in their family or social environment. In part 3 the pupils' experiencing preparing snacks themselves as fun was intended to strengthen the emotional aspect of eating behaviour. Altogether, cognitive information in part 1 was combined with skill training, operant conditioning, model learning and peer education in part 2 and 3.

\section{METHODS}

\section{Study Design}

The project was evaluated using a pre-/post-test research design without a control group. The study was reviewed and approved by the State Schools Authority of Lower Saxony, Germany.

\section{Participants and Recruitment}

Eighty-four classes from 40 different schools participated in the project "5-a-day for kids" during the selected time period of January 2011 to January 2012. Six fourth-grade classes were excluded from the evaluation due to administrative reasons. Furthermore, 9 classes refused to participate in the survey, mainly because the teachers did not consent to take over the handling of the questionnaires. Accordingly, the sample included 69 classes with 1,376 pupils, their parents and 69 teachers from 35 different schools. The majority, 65 classes, were from primary schools with third- and fourth-graders. The other four classes belonged to special education schools with higher class levels (5-7). The age ranged from 7 to 14 years.

\section{Instruments}

The data collection was performed with four different types of questionnaires. The parents' questionnaire collected socio-demographic data including size of household, country of origin, nationality, school education, professional training, and employment; moreover, it inquired about the availability of fruit and vegetables at home. The parents received their questionnaire before their child took part in "5-a-day for kids".

Information about the subject "nutrition" in the curriculum and further interventions already done in class were determined in the teacher questionnaire. Beyond that, the teachers were asked to describe their impressions about possible effects regarding the increase of fruit and vegetable consumption in school and to rate each part of the programme with school grades after the intervention. Additionally, they were asked for suggestions for improvement. 
The pupils' questionnaire included questions to test the topics covered during the programme as well as demographics (e.g. sex and age of the child, country of origin of both parents) and the child's self-reported weight and height. The children were additionally prompted to give grades for the project. This questionnaire was completed in the classroom in the presence of their teacher, who offered help if it was needed. The appropriateness of this method of collecting data was tested in a small pre-test. Both instruments (teacher and pupil questionnaire) were distributed after the intervention.

The Food Frequency Questionnaire (FFQ) “What do you eat?” for children, which had been applied in the German National Health Interview and Examination Survey for Children and Adolescents [Mensink and Burger, 2004] and had been validated in the EsKiMo Nutrition Module (a subsample of KiGGS) [Truthmann et al., 2011], was chosen to assess the long-term habitual food intake of the children [Hebert et al., 1995]. The FFQ inquired about the consumption frequency and portion sizes of approximately 50 main food groups during the previous four weeks [Mensink and Burger, 2004; Mensink et al., 2007b; Truthmann et al., 2011]. It aimed to focus parents on their children's total food intake, thus trying to prevent overestimation of the child's fruit and vegetable consumption. The questionnaire was structured as follows: in the first part, the parents were supposed to answer how often their child eats or drinks the respective food, food group or beverage. They could select from 10 options ranging from "never" to "more than 5 a day". In the second part, the parents were specifically asked about how much the child mostly eats or drinks of it. Answer categories were for example " $1 / 4$ (or less) portion", "1/2 portion", “1 portion", "2 portions", “3 (or more) portions” or other common units of consumption (glass, cup, plate, slice, tablespoon, etc.). For some items, exemplary photos were given to the parents to help them estimate the average size of the consumed portion (Figure 1) [Mensink and Burger, 2004; Mensink et al., 2007b; Truthmann et al., 2011]. To assess children's food consumption, it is a common method to measure portion sizes. One portion was set at $\sim 80$ g [Bere et al., 2006b; Ashfield-Watt, 2006; Reinaerts et al., 2008]. 
Figure 1: Extract of FFQ „What do you eat?” - Example for portion size (@ Robert Koch-Institute)

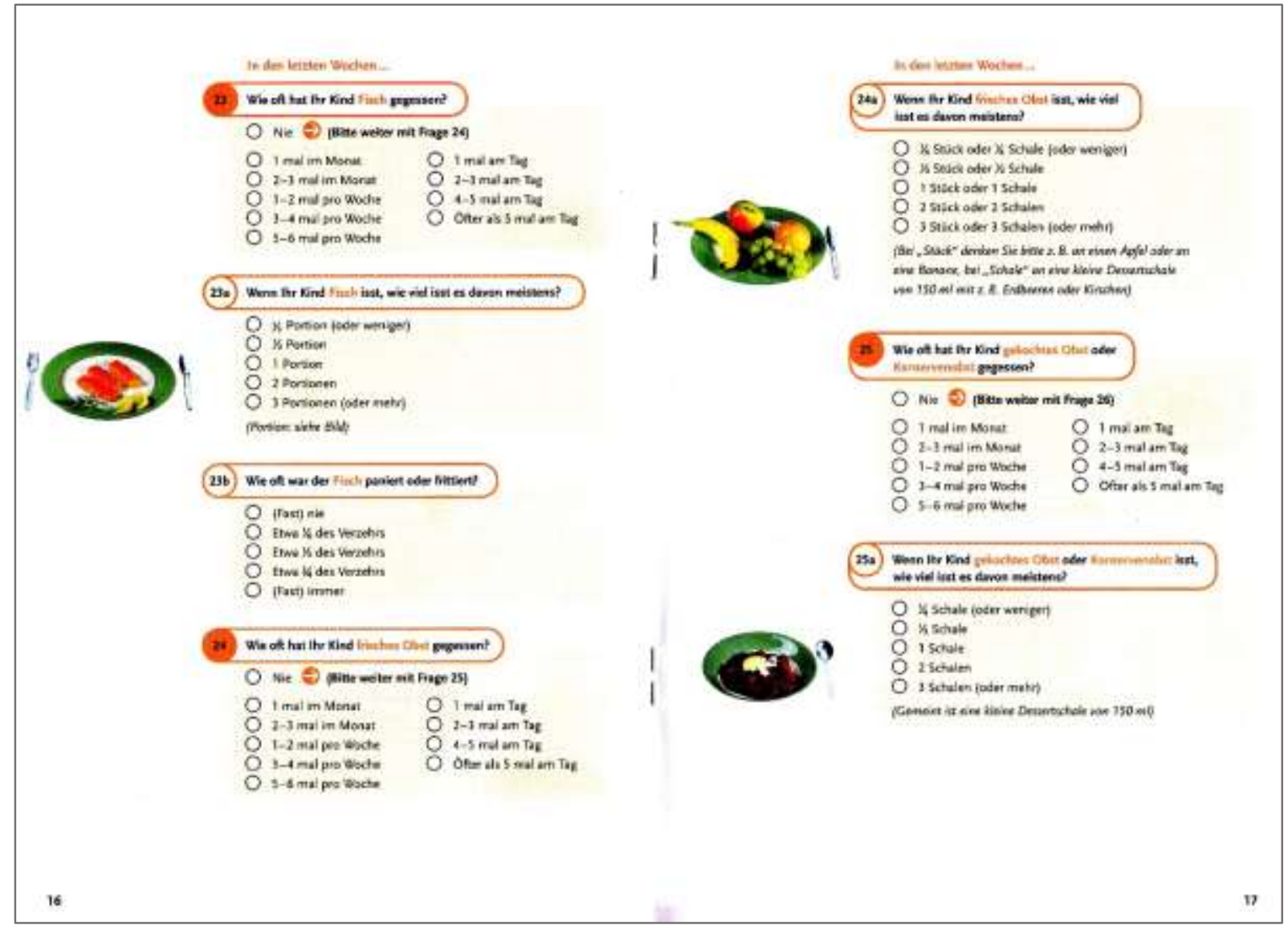

Each child's eating behaviour was recorded before intervention (baseline), one month (followup 1) and three months (follow-up 2) after intervention. The questionnaires were taken home by the children to be completed by one of their parents. All the questionnaires were distributed and returned via the school, and we had to rely on the goodwill and cooperation of the teachers involved to oversee this process. However, that was the only way to deal with; otherwise, the parents would not have been reachable.

\section{Data Analysis}

The data were analysed using the commercially available software Statistical Package for the Social Sciences (version 20.0, SPSS Inc., Chicago, IL, USA). Descriptive statistics and measures of association were used to summarise the demographics and outcome measures. Continuous variables are expressed as mean \pm standard deviation (SD); frequencies are expressed in percentages (\%). In addition, tests for the normal distribution of continuous measures were conducted. The Mann-Whitney U test was used for the comparison of the different outcome variables (e.g. sex, age, education and ethnicity). The mean daily intakes of fruit, vegetables and juice were calculated from the FFQs and changes between baseline and 
follow-up 1, baseline and follow-up 2, and between follow-up 1 and follow-up 2 were calculated. A Wilcoxon signed-rank test was conducted to explore the differences between the various food groups (especially fruit and vegetables). For this analysis, any participants who did not complete all three FFQs were left out. Furthermore, any FFQ, which showed an amount of more than $1,300 \mathrm{~g}$ of fruit and/or vegetables or more than $2,000 \mathrm{ml}$ of fruit juice, were excluded from this analysis, as it was unlikely that a child would consume such quantities [Fischer et al., 2011]. That concerned 44 children (in at least one of the three FFQs). In addition, participants with missing values for fruit, vegetable and/or juice consumption in at least one of the three FFQs were excluded. A probability value (p) of less than 0.05 was selected to indicate statistical significance.

\section{RESULTS}

Nine-hundred thirty FFQs were completed at baseline, while a total of 669 questionnaires were filled in at follow-up 1 and 545 FFQs were sent back at follow-up 2, giving response rates of $68 \%, 49 \%$ and $40 \%$, respectively. Four-hundred and forty children (respectively their parents) completed all three FFQs. All 5 instruments (pupils' and parent's questionnaires, three FFQs) were returned from 407 pupils.

The main characteristics of the study population are described in Table 1. 
Table 1. Demographic Characteristics of the Sample*

Pupil level data $(\mathrm{n}=979)$

Sex

$\operatorname{Girl}(\%)$

$496(50.7)$

Boy $(\%)$

483 (49.3)

Total (\%)

$979(100)$

Age (years) (SD)

$9.0(0.9)$

Age range (years)

$7.0-14.0$

Weight (kg) (SD)

$33.4(7.4)$

Height (m) (SD)

$1.4(0.1)$

Parent level data $(n=1.004)$

Household (SD)

$4.1(1.2)$

German nationality

Mother (\%)

$891(89.9)$

Father (\%)

$850(89.5)$

Maternal school education

HS (\%)

$152(15.8)$

Abitur (\%)

Paternal school education

HS (\%)

$214(23.5)$

Abitur (\%)

$220(24.1)$

Maternal professional training

Apprenticeship (\%)

$457(49.0)$

University degree (\%)

$102(10.9)$

Paternal professional training

Apprenticeship (\%)

$394(44.2)$

University degree (\%)

132 (14.8)

Maternal employment

Full-time (\%)

$140(14.7)$

Part-time (\%)

$549(57.7)$

Unemployed (\%)

$66(6.9)$

Paternal employment

Full-time (\%)

$793(87.5)$

Part-time (\%)

$41(4.5)$

Unemployed (\%)

42 (4.6)

School level data $(\mathrm{n}=41)$

Class size (SD)

$19.7(4.7)$

Number of children with migratory

background per class (SD)

Number of children with socially deprived

circumstances per class (SD)

$4.1(3.7)$

$\mathrm{HS}=$ certificate of completion of compulsory basic secondary education $\left(9^{\text {th }}\right.$ grade)

Abitur $=$ school-leaving examination usually taken at the end of the $13^{\text {th }}$ year

*Figures quoted are mean (standard deviation) or absolute number (\%).

According to the grades given by the pupils and teachers, the overall acceptance of the programme was good. Each part of the project was graded as shown in Figures 2 and 3. 
Figure 2. Students' Grades to Assess Parts 1-3 of the Project after Intervention (N=928)

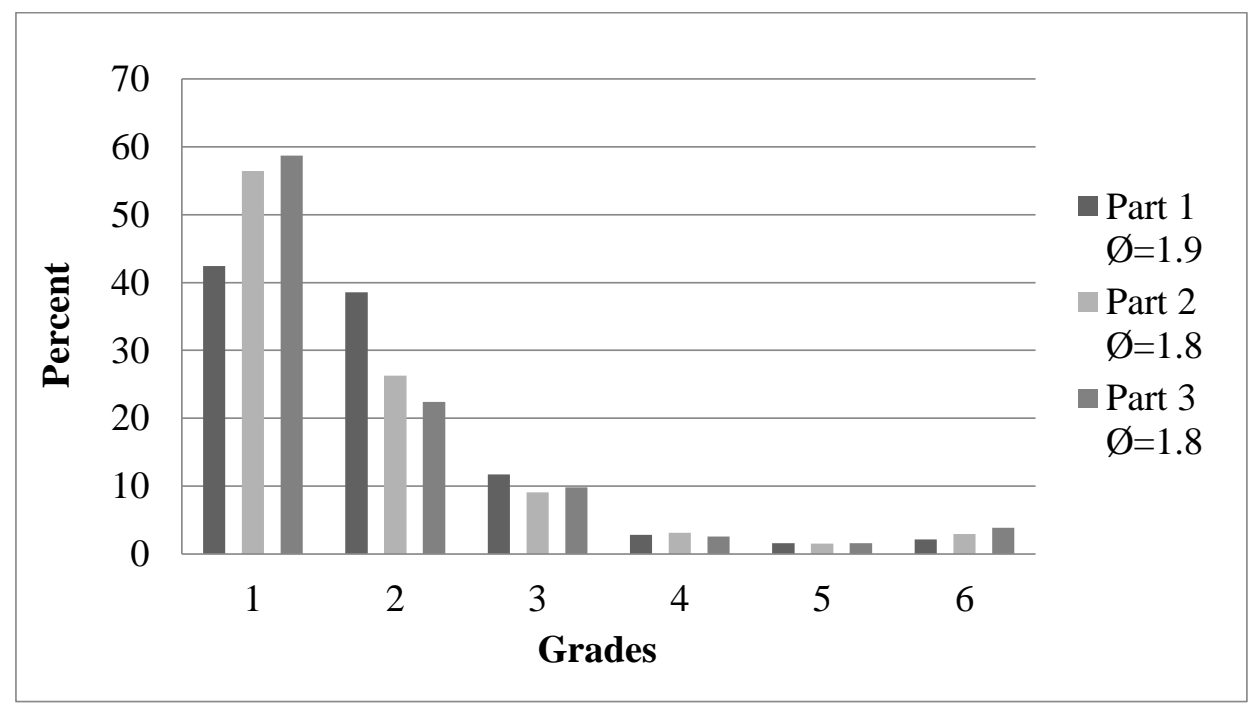

Figure 3. Teacher's Grades to Assess Parts 1-3 and the Overall Project after Intervention $(\mathrm{N}=39)$

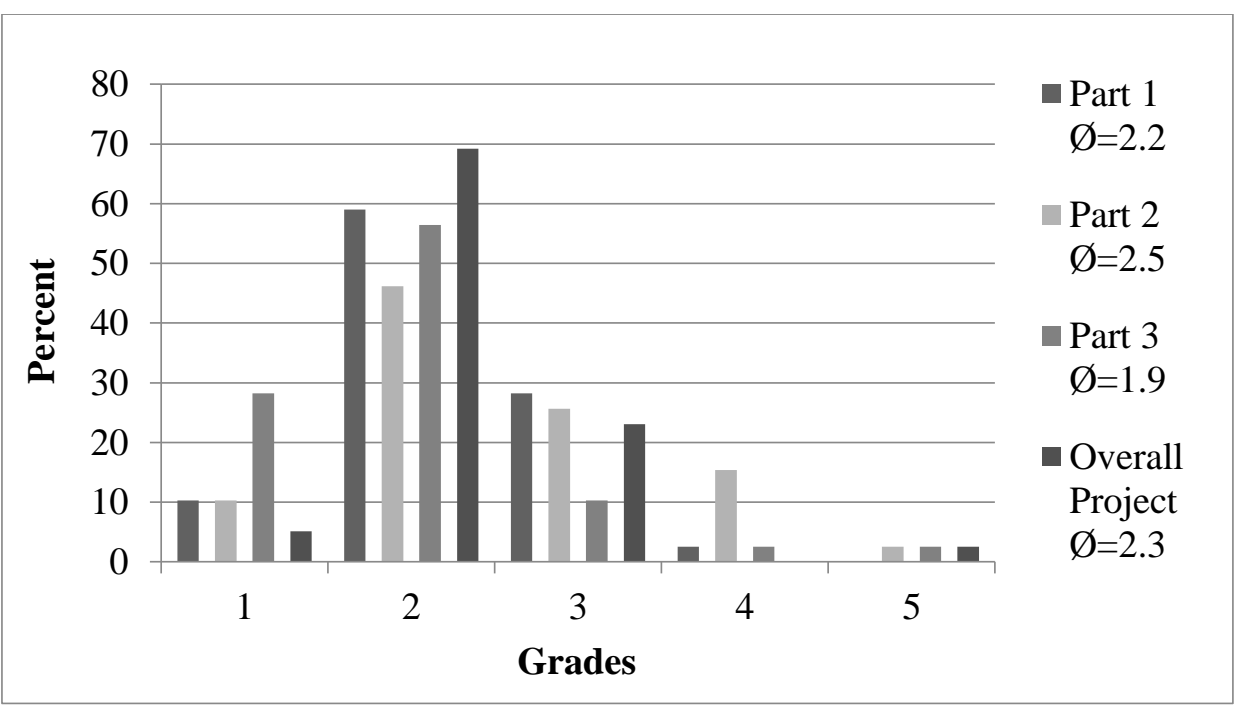

More than half of the teacher (54\%) made suggestions for improvement. 22\% suggested expanding the duration of the project or to repeating the intervention at regular intervals. Increasing the practical part of the programme was proposed by $10 \%$ of the teaching staff. Likewise, $10 \%$ suggested involving parents to enhance the effectiveness of the intervention. Fifteen percent made suggestions for improving the second part in the supermarket.

No steady increase could be observed for the intake of fruit and vegetables eaten throughout the day, neither at follow-up 1 nor at follow-up 2. In contrast, the intake of vegetables, fruit juice and the total amount of fruit/vegetables/juice showed a significant decline between baseline and follow-up 1 and between baseline and follow-up 2. Only fruit consumption rose 
temporarily between baseline and follow-up 1, though not significantly. These results are shown in Table 2.

Table 2. Intake of Fruit, Vegetables and Fruit Juices (in $\mathrm{g}$ or $\mathrm{ml}$ ) at the Three Points of Measurement $(\mathrm{n}=368)$

\begin{tabular}{|c|c|c|c|c|}
\hline Food & $\begin{array}{l}\text { Baseline } \\
\text { (B) }\end{array}$ & Follow-up 1 (FU1) & $\begin{array}{c}\text { Follow-up } 2 \\
\text { (FU2) }\end{array}$ & Significance $^{a}$ \\
\hline Fruit (g/day) & $248.1 \pm 231.7$ & $257.0 \pm 233.8$ & $225.9 \pm 215.5$ & ns; *; \#\# \\
\hline Vegetables (g/day) & $146.2 \pm 125.6$ & $138.7 \pm 117.6$ & $133.3 \pm 126.2$ & $\mathrm{~ns} ;{ }^{*}, \mathrm{~ns}$ \\
\hline Fruit juice (ml/day) & $249.6 \pm 344.6$ & $213.0 \pm 290.7$ & $192.6 \pm 276.6$ & $+; * * * ; \#$ \\
\hline $\begin{array}{l}\text { Total amount } \\
\text { fruit/vegetables/juice } \\
\text { (g/day) }\end{array}$ & $643.9 \pm 474.1$ & $608.7 \pm 421.7$ & $551.8 \pm 412.2$ & $\mathrm{~ns} ; * * * ; \# \# \#$ \\
\hline $\begin{array}{l}\text { All values reported are mean } \\
\mathrm{ns}=\text { not significant } \\
+\mathrm{p} \leq .05 \mathrm{~B} \text { vs. FU1 } \\
* \mathrm{p} \leq .05 \mathrm{~B} \text { vs. FU2 } \\
* * * \mathrm{p} \leq .001 \text { B vs. FU2 } \\
{ }^{\mathrm{a}} \text { calculated using the Wilcox }\end{array}$ & $\begin{array}{l}\text { standard deviation } \\
\# \mathrm{p} \leq .05 \text { FU1 vs. FU2 } \\
\# \# \mathrm{p} \leq .01 \mathrm{FU} 1 \text { vs. FU2 } \\
\# \# \# \mathrm{p} \leq .001 \text { FU1 vs. FU2 } \\
\text { signed-ranked test }\end{array}$ & & & \\
\hline
\end{tabular}

The results of the analysis were similar independently of whether the estimated changes in fruit and vegetable consumption were measured in grams or portions. The fruit intake showed a minimal increase of less than 0.1 portions after one month but then declined about 0.2 portions at follow-up 2. The consumption of vegetables diminished by about 0.1 portions between baseline and follow-up 2. The details are presented in Table 3.

Table 3. Intake of Fruit and Vegetables (in Portions per Day) $(\mathrm{n}=395)$

\begin{tabular}{|c|c|c|c|c|}
\hline Food & $\begin{array}{c}\text { Baseline } \\
\text { (B) }\end{array}$ & $\begin{array}{c}\text { Follow-up } 1 \\
\text { (FU1) }\end{array}$ & $\begin{array}{c}\text { Follow-up } 2 \\
\text { (FU2) }\end{array}$ & Significance $^{\mathrm{a}}$ \\
\hline Fruit & $1.67 \pm 1.57$ & $1.71 \pm 1.57$ & $1.51 \pm 1.45$ & ns; *;\#\# \\
\hline Vegetables & $1.21 \pm 1.16$ & $1.12 \pm 1.02$ & $1.07 \pm 1.05$ & ns; *; \# \\
\hline $\begin{array}{l}\text { All values reported ar } \\
\mathrm{ns}=\text { not significant } \\
* \mathrm{p} \leq .05 \mathrm{~B} \text { vs. FU2 } \\
{ }^{\mathrm{a}} \text { calculated using the }\end{array}$ & $\begin{array}{l}\text { leviation } \\
\text { FU1 vs. FU2 } \\
1 \text { FU1 vs. FU2 } \\
\text { k test }\end{array}$ & & & \\
\hline
\end{tabular}

With regard to the fruit and vegetable consumption, no significant differences were found based on sex or class level (results not shown). No significant interrelationship between the valuation of the programme by the children and their fruit and vegetable intake could be observed (results not shown).

An increased intake of fruit and vegetables of the children was associated with a higher education of the parents (Table 4). Children whose parents had "Abitur" (school-leaving examination usually taken at the end of the 13th year) ate significantly more fruit and vegetables than pupils whose parents got a certificate of completion of compulsory basic secondary 
education (9th grade). The fruit and vegetable intake also correlated with the parental professional training. With children, whose parents possessed a university degree a significantly higher consumption could be observed.

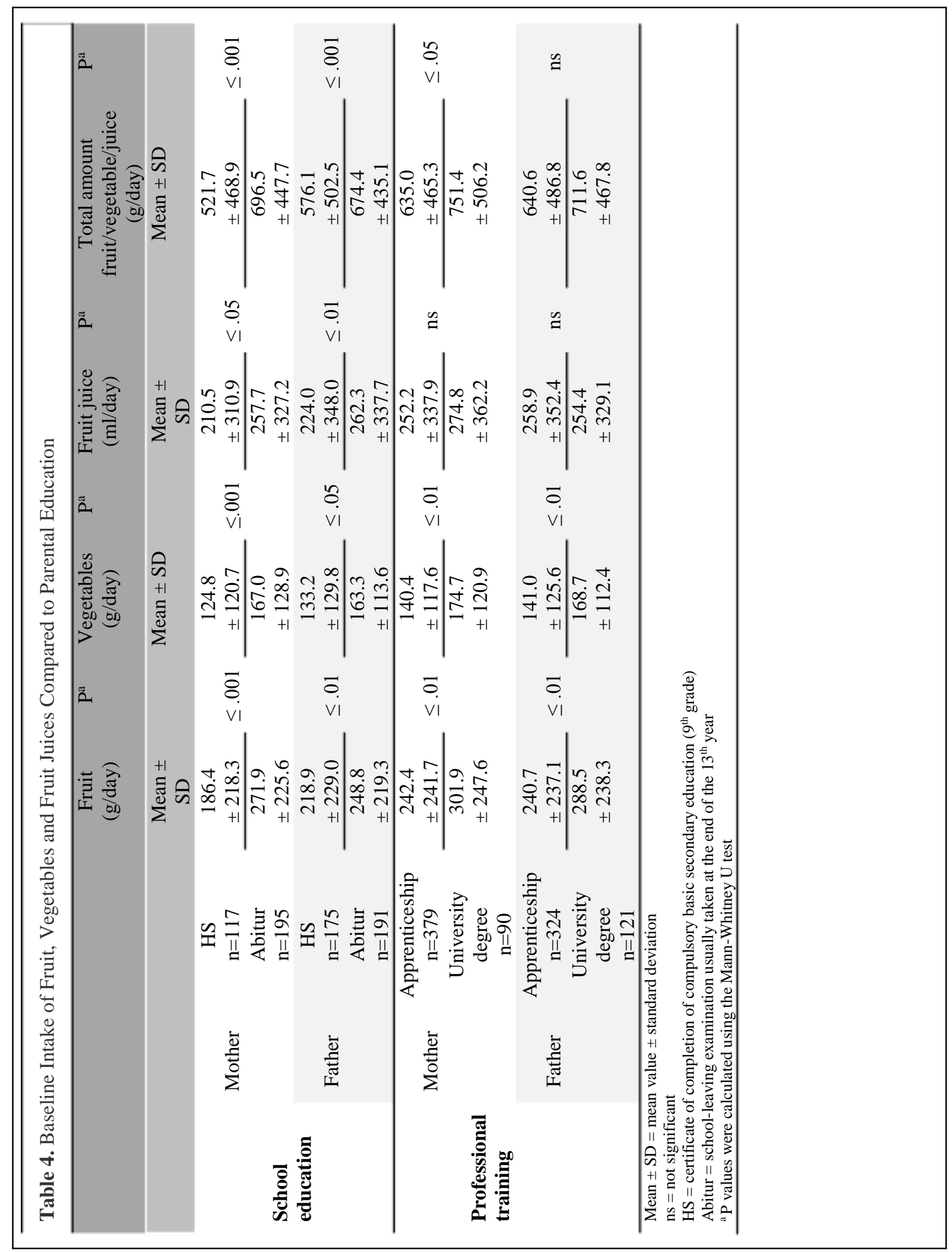




\section{DISCUSSION}

The purpose of this study was to investigate the acceptance and the short- and intermediateterm effects of the school-based nutrition programme "5-a-day for kids".

The acceptance of the intervention by both teachers and pupils was high. These results confirmed our presumption that the acceptability is good, based on the great demand and interest for the project over years as well as the positive feedback. Nevertheless, no significant change in behaviour leading to the requested increase of fruit and vegetable intake was measured.

Altogether, the average fruit and vegetable intake at baseline was clearly higher than in comparable studies. The difference amounted to about 50 gram for vegetables and between 130 and 140 gram for fruit [Robert Koch-Institut, 2006; Mensink et al., 2007a]. In our cohort, at baseline the recommended daily amount of vegetables was consumed by only $21 \%$ of the girls and $20 \%$ of the boys, whereas $40 \%$ of the female pupils and $38 \%$ of the male pupils achieved the recommendation for fruit. In comparison to the Eating Study as KiGGS Module, the percentage of children eating vegetables was reported to be three times higher and the percentage of those eating fruit was about two times higher [Mensink et al., 2007b]. A possible reason for this result could be the overestimation of fruit and vegetable intake due to social desirability [Hebert et al., 1995] because the parents completed the first FFQ and their questionnaire (in which the first five questions deal with fruit and vegetables) at the same time. A further explanation could be that parents who have a high level of concern with their children's diet are more likely to have children who eat fruit and vegetables and are more likely to answer all FFQs. If so, this could explain the high per cent of children who eat the recommended levels of fruit and vegetables and it might also explain the negative results children who live in homes where healthy diet is already stressed are unlikely to benefit further from brief intervention in school.

In addition, our results confirmed the strong association between children's fruit and vegetable intake and the socioeconomic status (SES) based on parental education and parental professional training, which has been well documented in the existing literature [Rasmussen et al., 2006]. In general, the strength of correlation seemed to be stronger between the intake and maternal school education than paternal school education. Further personal, social and 
environmental determinants of children's fruit and vegetable consumption like knowledge, availability at home and in school are discussed elsewhere [Mittmann et al., 2014].

\section{Limitations}

The results of this analysis should be approached cautiously since there are some limitations. The intervention was planned almost 15 years ago by an advertising agency. That is why it was not based on behaviour change theory but on marketing strategies. The following aspects were given priority:

- Profiling and image building of the retail segment within a young target group

- Sales increase of fruit and vegetables

- Establishing a network between schools and retail segment connected by a neutral institution or nutrition expert

- Creating proximity to potential customers

- Reducing mutual reservations to fruit and vegetables (of children) and increasing the appreciation

In contrast to other "5-a-day" interventions, which were randomized or non-randomized controlled trials, this study did not investigate a control group but used a pretest-posttest design with a big sample size to measure the effects. Due to the study design, which was based on the above mentioned concept of carrying out approximately 100 projects a year by one person, and the fact that the data collection was spread over 15 months, it would have been difficult to identify a control group that was not subjected to various influences regarding nutrition education.

With regard to the selected method of data collection, the major disadvantages of the FFQ should be mentioned: only estimates of the average size of the consumed portion were taken [Mensink and Burger, 2004] and parents are not reliable reporters of their children's out-ofhome food intake [Livingstone and Robson, 2000]. Furthermore, there is a risk of under- or over-reporting due to the nature of self-reported data [Livingstone and Robson, 2000]. There was a large dropout between the baseline measurement and the first and second follow-up. Possible reasons could be that many study participants came from disadvantaged social backgrounds, had an inadequate knowledge of the German language or that the workload for the parents was too high. We limited the data collection to keep the workload moderate for the 
participants. That is the reason why our findings do not allow conclusions why the intervention does not appear to work.

\section{Implications for Practice}

Based on our results, it can be concluded that improvements to the described intervention are necessary to enhance its effectiveness. Basically there is a great body of evidence for the effectiveness of school-based nutrition interventions that combine availability of fruit and vegetables in schools with a nutrition education curriculum and parental involvement [Blanchette and Brug, 2005; Van Cauwenberghe et al., 2010]. As suggested by the teacher, promising elements like providing a prolonged intervention to ensure the effects are maintained, improving practical skills or incorporating and maintaining family involvement, which have already shown significant effects in recent fruit and vegetable promoting programmes [Burchett, 2003], could be included in a revised programme. As our results confirmed, availability at home and school is a strong predictor for children's fruit and vegetable intake [Blanchette and Brug, 2005; Krølner et al., 2011; Mittmann et al., 2014]. It may be wise to focus on this together with providers of the EU school fruit programme. The duration of the project could be increased; however, such an increase would lead to a decrease in the number of pupils reached each year or would lead to a rise in costs, if more staff were employed. If the intervention involved a greater length of time then its implementation in schools might become more difficult due to interfering more with the school's standard curriculum.

Altogether, all these results have to be discussed especially with all federal Cancer Societies which still offer "5-a-day-for-kids" to decide about continuation or improvements (taking into account moderate costs for programme provider, simple implementation, broad acceptance and support by children, parents, teacher and supermarket staff).

Our findings confirmed the assumption that not only activities regarding behaviour change, but also improvements regarding environmental factors are required. In general, an institutional anchoring is useful; healthy eating has to become a basic philosophy in a comprehensive school health programme. In practice, this means implementing a school subject "nutrition" which is mainly practical-orientated during the whole educational process, well informed and trained faculty and cafeteria staff, equipment (kitchen), offering healthy food at cafeteria, vending machines and school kiosk, teacher as models. For this purpose, the policy is called upon to create the necessary framework and invest in education. 


\section{Implications for Future Research}

Further research to identify promising elements of changing children's eating behaviour is needed because the results of school based nutrition intervention studies regarding their effectiveness are inconsistent. Several multicomponent programmes seemed to be effective in prior investigations [Howerton et al., 2007; Van Cauwenberghe et al., 2010] whereas predominantly knowledge-based methods have shown no increasing in fruit and vegetable intake in recent studies [Kipping et al., 2014]. Furthermore, research should focus on the questions how to make healthy eating more attractive and how to improve the availability of fruit and vegetables especially in families with a low socioeconomic status. Scientific evidence is required to develop target-group-specific interventions.

\section{Conclusion}

Although the findings of our study documented no sustainable behaviour change, the acceptance and implementation of the short school-based nutrition intervention was well assessed. Nevertheless, our insights disclosed the constraints of such a project; it could only be one of several components which make healthy eating more attractive for children. The short nutrition intervention "5-a-day-for-kids" offered by the Cancer Society of Lower Saxony could not compensate for unfavourable education primarily by parents and other role models over years. It became clear that this short-term intervention is limited regarding to its effectiveness and that further measures and improvements are required. 


\section{REFERENCES}

Alexy, U., Clausen K. and Kersting, M. (2008), "Die Ernährung gesunder Kinder und Jugendlicher nach dem Konzept der Optimierten Mischkost", ("The optimised mixed diet: a concept for nutrition of healthy children and adolescents"), Ernährungs Umschau, Vol. 3, pp. 168-177.

Ashfield-Watt, P.A.L. (2006), 'Fruits and vegetables, 5+ a day: are we getting the message across?“, Asia Pacific Journal of Clinical Nutrition, Vol. 15 No. 2, pp. 245-252.

Baranowki, T., Davis, M., Resnicow, K., Baranowski, J., Doyle, C., Lin, L.S., Smith, M. and Wang, D.T. (2000), "Gimme 5 fruit, juice, and vegetables for fun and health: outcome evaluation", Health Education \& Behavior, Vol. 27 No. 1, pp. 96-111.

Bere, E., Veierød, M.B., Bjelland, M. and Klepp, K.-I. (2006a), "Free school fruit - sustained effect 1 year later", Health Education Research, Vol. 21 No. 2, pp. 268-275.

Bere, E., Veierød, M.B., Bjelland, M. and Klepp, K. (2006b), “Outcome and process evaluation of a Norwegian school-randomized fruit and vegetable intervention: fruits and vegetables make the marks (FVMM), Health Education Research, Vol. 21 No. 2, pp. 258-267.

Bere, E., Veierød, M.B., Skare, Ø. and Klepp, K. (2007), "Free school fruit - sustained effect three years later", International Journal of Behavioral Nutrition and Physical Activity, Vol. 4. No.1.

Blanchette, L. and Brug, J. (2005), "Determinants of fruit and vegetable consumption among 6-12-year-old children and effective interventions to increase consumption", Journal of Human Nutrition and Dietetics, Vol. 18 No. 6, pp. 431-443.

Boeing, H., Bechthold, A., Bub, A., Ellinger, S., Haller, D., Kroke, A., Leschik-Bonnet, E., Müller, M.J., Oberritter, H., Schulze, M., Stehle, P. and Watzl, B. (2012), “Critical review: vegetables and fruit in the prevention of chronic diseases", European Journal of Nutrition, Vol. 51 No. 6, pp. 637-663. 
Bundeszentrale für gesundheitliche Aufklärung (2009), Unterrichtsmaterialien zur Ernährungserziehung in der Grundschule - eine bundesweite Bestandsaufnahme, (Teaching materials for nutritional education in primary schools - a nationwide survey), Gesundheitsförderung konkret, Band 11, Bundeszentrale für gesundheitliche Aufklärung, Köln.

Burchett, H. (2003), "Increasing fruit and vegetable consumption among British primary schoolchildren: a review", Health Education, Vol. 103 No. 2, pp. 99-109.

De Sa, J. and Lock, K. (2007), "School-based fruit and vegetable schemes: a review of the evidence", London School of Hygiene and Tropical Medicine, London, available at: http://ec.europa.eu/agriculture/sfs/documents/de_sa_lock_en.pdf (accessed 13 January 2016).

Deutsche Gesellschaft für Ernährung e.V. (2008), Ernährungsbericht 2008, (Nutrition Report 2008), Deutsche Gesellschaft für Ernährung e.V., Bonn.

Domel, SB., Baranowski, T., Davis, H., Thompson, WO., Leonard, SB., Riley, P., Baranowski, J., Dudovitz, B. and Smyth, M. (1993), "Development and evaluation of a school intervention to increase fruit and vegetable consumption among 4th and 5th grade students", Journal of Nutrition Education and Behavior, Vol. 25 No. 6, pp. 345-349.

Evans, C.E.L., Christian, M.S., Cleghorn, C.L., Greenwood, D.C. and Cade, J.E. (2012), "Systematic review and meta-analysis of school-based interventions to improve daily fruit and vegetable intake in children aged 5 to 12 y", The American Journal of Clinical Nutrition, Vol. 96 No. 4, pp. 889-901.

Fischer, C., Brug, J., Tak, N.I., Yngve, A. and te Velde, S.J. (2011), “Differences in fruit and vegetable intake and their determinants among 11-year-old schoolchildren between 2003 and 2009”, International Journal of Behavioral Nutrition and Physical Activity, 8:141.

Gerhards, J. and Rössel, J. (2003), "Das Ernährungsverhalten Jugendlicher im Kontext ihrer Lebensstile - Eine empirische Studie", ("The nutrition behaviour of adolescents in the context of their lifestyle - an empirical study"), Forschung und Praxis der Gesundheitsförderung, Band 20, Bundeszentrale für gesundheitliche Aufklärung, Köln. 
Hastings, G., Stead, M., McDermott, L., Forsyth, A., MacKintosh, A.M., Rayner, M., Godfrey, C., Caraher, M. and Angus, K. (2003), "Review of research on the effects of food promotion to children - final report", Centre for Social Marketing, Glasgow, available at: http://tna.europarchive.org/20110116113217/http:/www.food.gov.uk/multimedia/ pdfs/foodpromotiontochildren1.pdf (accessed 13 January 2016).

Hebert, J.R., Clemow, L., Pbert, L., Ockene, I.S. and Ockene J.K. (1995), “Social desirability bias in dietary self-report may compromise the validity of dietary intake measures", International Journal of Epidemiology, Vol. 24 No. 2, pp. 389-398.

Heimendinger, J. and Van Duyn, M.A.S. (1995), "Dietary behavior change: the challenge of recasting the role of fruit and vegetables in the American diet", The American Journal of Clinical Nutrition, Vol. 61 No. 6, pp. 1397S-1401S.

Howerton, M.W., Bell, B.S., Dodd, K.W., Berrigan, D., Stolzenberg-Solomon, R. and Nebeling L. (2007), "School-based nutrition programs produced a moderate increase in fruit and vegetable consumption: meta and pooling analyses from 7 studies", Journal of Nutrition Education and Behavior, Vol. 39 No. 4, pp. 186-196.

Hyson, DA. (2011), "Fruits, vegetables, and health: a scientific overview", Wilmington, DE: Produce for Better Health Foundation, available at: http://www.pbhfoundation.org/pdfs/ about/res/pbh_res/PBH_Health_Benefit_Review.pdf (accessed 14 January 2016).

Kipping, R.R., Howe, L.D., Jago, R., Campbell, R., Wells, S., Chittleborough, C.R., Mytton, j., Noble, S.M., Peters, T.J. and Lawlor, D.A. (2014), "Effect of intervention aimed at increasing physical activity, reducing sedentary behaviour, and increasing fruit and vegetable consumption in children: active for live year 5 (AFLY5) school based cluster randomised controlled trial”, British Medical Journal, 348:g3256.

Klepp K.I., Pérez-Rodrigo, C., De Bourdeaudhuij, I., Due, P., Elmadfa, I., Haraldsdóttir, J., König, J., Sjöström, M., Thórsdóttir, I., Vaz de Almeida, M.D., Yngve, A. and Brug, J. (2005), "Promoting fruit and vegetable consumption among European schoolchildren: rationale, conceptualization and design of the pro children project", Annals of Nutrition \& Metabolism, Vol. 49 No. 4, pp. 212-220. 
Krølner, R., Rasmussen, M., Brug, J., Klepp, K.-I., Wind, M. and Due, P. (2011), “Determinants of fruit and vegetable consumption among children and adolescents: a review of the literature. Part II: qualitative studies", International Journal of Behavioral Nutrition and Physical Activity, 8:112.

Livingstone, M.B.E. and Robson, P.J. (2000), "Measurement of dietary intake in children”, Proceedings of the Nutrition Society, Vol. 59 No. 2, pp. 279-293.

Mensink, G.B.M. and Burger, M. (2004), “Was isst du? Ein Verzehrshäufigkeitsfragebogen für Kinder und Jugendliche", ("What do you eat? A food frequency questionnaire for children and adolescents"), Bundesgesundheitsblatt - Gesundheitsforschung - Gesundheitsschutz, Vol. 47 No. 3, pp. 219-226.

Mensink, G.B.M., Heseker, H., Richter, A., Stahl, A. and Vohmann, C. (2007a), "Forschungsbericht. Ernährungsstudie als KiGGS-Modul (EsKiMo)", ("Research report. Nutrition study as KiGGS-module (EsKiMo)"), Robert KochInstitut/Universität Paderborn, Bonn, available at: https://www.bmel.de/SharedDocs/Downloa ds/Ernaehrung/EsKiMoStudie.pdf?__blob=publicationFile (accessed 13 January 2016).

Mensink, G.B.M., Kleiser, C. and Richter, A. (2007b), "Lebensmittelverzehr bei Kindern und Jugendlichen in Deutschland. Ergebnisse des Kinder- und Jugendgesundheitssurveys (KiGGS)", ("Food intake of children and adolescents in Germany. Results of the German national health interview and examination survey for children and adolescents (KiGGS)“"), Bundesgesundheitsblatt - Gesundheitsforschung - Gesundheitsschutz, Springer Medizin Verlag, Vol. 50, pp. 609-623.

Mittmann, S., Austel, A. and Ellrott, T. (2014), "Welche Faktoren sind mit dem Obst- und Gemüseverzehr von Grundschülern assoziiert", ("Determinants of fruit and vegetable consumption in primary schoolchildren”), Ernährungs Umschau, Vol. 61 No. 8, pp. 124-129.

Perry, C.L., Bishop, D.B., Taylor, G., Murray, D.M., Warren Mays, R., Dudovitz, B.S., Smyth, M. and Story, M. (1998), "Changing fruit and vegetable consumption among children: the 5-aday power plus program in St. Paul, Minnesota”, American Journal of Public Health, Vol. 88 No. 4, pp. 603-609. 
Potter J.D., Finnegan, J.R., Guinard, J.-X., Huerta, E.E., Kelder, S.H., Kristal, A.R., Kumanyika, S., Lin, R., McAdams Motsinger, B., Prendergast, F.G. and Sorensen, G. (2000), 5 a day for better health - program evaluation report, Bethesda, MD: National Institutes of Health, National Cancer Institute, NIH Publication No. 01-4904, available at: http://www.scgcorp.com/docs/5_a_Day_Booklet_sm.pdf (accessed 13 January 2016).

Rasmussen, M., Krølner, R., Klepp, K.I., Lytle, L., Brug, J., Bere, E. and Due, P. (2006), "Determinants of fruit and vegetable consumption among children and adolescents: a review of the literature. Part I: quantitative studies”, International Journal of Behavioral Nutrition and Physical Activity, 3:22.

Reinaerts, E., Crutzen, R., Candel, M., De Vries, N.K. and De Nooijer, J. (2008), “Increasing fruit and vegetable intake among children: comparing long-term effects of a free distribution and a multicomponent program", Health Education Research, Vol. 23 No. 6, pp. 987-996.

Robert Koch-Institut (2006), "Erste Ergebnisse der KiGGS-Studie zur Gesundheit von Kindern und Jugendlichen in Deutschland", ("First results of the German national health interview and examination survey for children and adolescents (KiGGS)“), Robert Koch-Institut, Berlin, available at: http://www.rki.de/DE/Content/Gesundheitsmonitoring/Studien/Kiggs/Basiserhebung/Ergebnisbroschüre.pdf?_blob=publicationFile (accessed 13 January 2016).

Roberts, B.P., Blinkhorn, A.S. and Duxbury, J.T. (2003), "The power of children over adults when obtaining sweet snacks", International Journal of Paediatric Dentistry, Vol. 13 No. 2, pp. 76-84.

Truthmann, J., Mensink, G.B.M. and Richter, A. (2011), "Relative validation of the KiGGS food frequency questionnaire among adolescents in Germany", Nutrition Journal, 10:133.

Van Cauwenberghe, E., Maes, L., Spittaels, H., van Lenthe, F.J., Brug, J., Oppert, J.-M. and De Bourdeaudhuij, I. (2010), "Effectiveness of school-based interventions in Europe to promote healthy nutrition in children and adolescents: systematic review of published and 'grey' literature", British Journal of Nutrition, Vol. 103 No. 6, pp. 781-797. 
Behavioural Effects of a Short School-Based Fruit and Vegetable Promotion Programme: 5-a-Day for Kids

World Health Organization (2003), "Diet, Nutrition and the Prevention of Chronic Diseases: Report of a joint WHO/FAO Expert Consultation", Geneva, 28 January - 1 February 2002, World Health Organization, WHO Technical Report Series No. 916, available at: http://whqlibdoc.who.int/trs/who_TRS_916.pdf (accessed 18 October 2012). 
Welche Faktoren sind mit dem Obst- und Gemüseverzehr von Grundschülern assoziiert?

Autoren: Silke Mittmann, Anja Austel, Thomas Ellrott

Veröffentlicht bei Ernährungs Umschau, 2014: 61, M422-M427,

DOI: $10.4455 / e u .2014 .022$

Mit freundlicher Genehmigung der Umschau Zeitschriftenverlag GmbH, Wiesbaden. 


\section{Zusammenfassung}

Kinder im Grundschulalter essen im Mittel weniger Obst und Gemüse als deutsche Fachinstitutionen empfehlen. Nicht alle Strategien zur Förderung des Obst-und Gemüseverzehrs sind erfolgreich. Zur Konzeptionierung und Planung von Interventionen im Setting Schule ist die Kenntnis wesentlicher Determinanten des Obst- und Gemüseverzehrs hilfreich. Um solche Determinanten zu identifizieren, wurde eine Erhebung an Grund-/ Förderschulen in Niedersachsen mit 1376 Kindern (mittleres Alter 9 Jahre) durchgeführt. Dazu wurden Schüler und Eltern mittels Fragebögen befragt. Der reale Verzehr wurde mithilfe des validierten Fragebogens zur Erfassung der Verzehrhäufigkeit „Was isst Ihr Kind?“ des RobertKoch-Instituts erhoben. Die Ergebnisse zeigen, dass Kinder, die täglich im Elternhaus Obst und Gemüse angeboten bekommen bzw. Obst und Gemüse mit in die Schule nehmen, deutlich mehr davon essen als Kinder, denen diese Lebensmittel nicht zur Verfügung stehen. Die kurzzeitige Intervention in der Schule zur Verbesserung des Wissens der Kinder über den gesundheitlichen Nutzen von Obst und Gemüse führte nicht zu einer Steigerung des Verzehrs dieser Lebensmittel. Daraus lässt sich ableiten, dass die Verfügbarkeit von Obst und Gemüse einen entscheidenden Einfluss auf den Verzehr hat. Interventionen, die zu einem häufigeren Angebot von Obst und Gemüse im Elternhaus, in der Frühstuicksbox oder in der Schule führen, erscheinen erfolgversprechend.

Schlüsselwörter: Obst, Gemüse, Verzehr, Ernährungsbildung, Kinder, Ernährungsverhalten 


\section{Einleitung}

Aktuelle Daten der KiGGS- und EsKiMo-Studie [1-3] belegen, dass Kinder in Deutschland zu geringe Mengen an Obst und Gemüse zu sich nehmen und die Empfehlungen des Forschungsinstituts für Kinderernährung (FKE) nicht erreicht werden. Um effektive schulische Interventionen zu konzipieren, die den Obst-und Gemüseverzehr erhöhen, müssen die Rahmenbedingungen, die das kindliche Essverhalten beeinflussen, berücksichtigt werden.

\section{Ernährungsverhalten im Kindesalter}

Das kindliche Ernährungsverhalten wird von einer Vielzahl von Faktoren aus dem persönlichen Bereich, dem sozialen Umfeld sowie durch die prinzipielle Verfügbarkeit beeinflusst $[4,5]$ • Tabelle 1 gibt einen Überblick über Einflussgrößen auf den Obst- und Gemüseverzehr.

Tabelle 1: Einflussfaktoren auf den Obst- und Gemüseverzehr bei Kindern [5]

\begin{tabular}{|c|c|c|}
\hline Persönliche Faktoren & Soziale Umweltfaktoren & $\begin{array}{l}\text { Physische Verfügbarkeit von } \\
\text { O+G }\end{array}$ \\
\hline Wissen & $\begin{array}{l}\text { Vorbildfunktion von Eltern, } \\
\text { Großeltern, Freunden }\end{array}$ & Verfügbarkeit zu Hause \\
\hline Einstellungen & $\begin{array}{l}\text { aktive elterliche Ermunterung } \\
\text { zum Verzehr }\end{array}$ & Verfügbarkeit in der Schule \\
\hline (Geschmacks-)Vorlieben & $\begin{array}{l}\text { Familienregeln, Aufforderung } \\
\text { zum Verzehr }\end{array}$ & Verfügbarkeit bei Freunden \\
\hline $\begin{array}{l}\text { Bevorzugung von } \\
\text { Lebensmitteln }\end{array}$ & $\begin{array}{l}\text { Erlaubnis (z.B. Menge selbst zu } \\
\text { bestimmen) }\end{array}$ & \\
\hline Selbstwirksamkeit & $\begin{array}{l}\text { elterliche Fähigkeiten zur } \\
\text { Zubereitung }\end{array}$ & \\
\hline $\begin{array}{l}\text { wahrgenommene Hindernisse } \\
\text { (Hungergefühl, Uhrzeit) }\end{array}$ & $\begin{array}{l}\text { Mitgabe von Obst und Gemüse } \\
\text { in Schule }\end{array}$ & \\
\hline $\mathrm{O}=$ Obst $; \mathrm{G}=\mathrm{C}$ & & \\
\hline
\end{tabular}

Determinanten, die einen großen Einfluss speziell auf den Obst- und Gemüseverzehr ausüben, sind Geschmacksvorlieben, Verfügbarkeit im Elternhaus und im Lebensumfeld, elterlicher Obst- und Gemüsekonsum (Vorbildfunktion), Kenntnisse über empfohlene Verzehrmengen, Portionsgrößen und Mahlzeitenstruktur, aber auch demografische Aspekte wie Alter, Geschlecht und sozioökonomischer Status [6-9]. Eine gesundheitsförderliche Ernährungsweise ist im Kindes- und Jugendalter von besonderer Bedeutung, da in diesem Lebensabschnitt die Grundlage für das spätere Ernährungsverhalten gelegt wird [9, 10]. Die Essgewohnheiten entwickeln sich insbesondere in der frühen Kindheit und bleiben i. d. R. auch im Erwachsenenalter konstant [7]. Es besteht eine enge Korrelation zwischen Eltern und Kindern bezüglich der Lebensmittelakzeptanz, dem Wissen, den Vorlieben, dem Verzehr sowie der 
Bereitschaft, neue Nahrungsmittel zu probieren [7]. Vorlieben für einzelne Lebensmittel werden u. a. davon bestimmt, wie häufig diese den Kindern angeboten werden, ob sie sie also „gewöhnt" sind (,mere exposure effect“) [7, 9, 11]. Gerade Mütter spielen bei der Ernährungserziehung auch heutzutage noch eine herausragende Rolle. Sie kochen die Gerichte bzw. servieren die Obst- und Gemüsesorten, die sie selbst gerne mögen und geben so ihre Geschmacksvorlieben, aber auch Abneigungen und allgemeine Gewohnheiten weiter [9]. Bei Kindern, deren Eltern ein gesundes Ernährungsverhalten durch das Angebot einer vielseitigen Obst- und Gemüseauswahl fördern, ist ein höherer Konsum dieser Lebensmittel zu verzeichnen. Zudem ist eine erhöhte Experimentierfreudigkeit gegenüber neuen, unbekannten Lebensmitteln zu beobachten [7-9].

\section{Setting (Grund-)Schule}

Neben den Eltern beeinflussen weitere Personen (Großeltern, Geschwister, Freunde) [7], aber auch Bildungsinstitutionen (Kindertageseinrichtungen, Schule) und Umwelteinflüsse wie Fernsehen/Werbung das kindliche Ernährungsverhalten. Gerade Bildungseinrichtungen tragen in den letzten Jahren immer mehr zur Ernährungsbildung von Kindern bei. Durch die wachsende Bedeutung des Pausenfrühstücks, v. a. aber durch den Ausbau von Ganztagsschulen und -betreuungseinrichtungen mit dem vermehrten Angebot von Mittagsverpflegung nimmt der Einfluss der Bildungsinstitutionen auf die Ernährungsbildung der Kinder zu [12]. Die Institution Schule ist besonders geeignet, ernährungsbezogene Inhalte und Handlungsweisen zu vermitteln, da - bedingt durch die Schulpflicht - die Kinder aller sozialen Schichten erreicht werden, die Schülerinnen und Schüler einen Großteil ihrer Zeit in der schulischen Umgebung verbringen und mindestens eine Mahlzeit dort einnehmen [13,14]. Durch Interventionen im Bereich Ernährungsbildung können über die Kinder dann wiederum die Eltern erreicht werden [15]. Die Zahl gesundheitsfördernder Programme externer Institutionen (z.B. „Klasse 2000“, aid-Ernährungsführerschein, Aktionen des Landfrauenverbandes etc.) für den Unterricht in der Schule wächst. Seit 2002 bietet die Niedersächsische Krebsgesellschaft e.V. für interessierte Grundschulen das Projekt „5 am Tag für Kids“ an. Ziel der dreistündigen Intervention ist die Steigerung des Obst- und Gemüseverzehrs von Dritt- und Viertklässlern in Niedersachsen.

\section{Fragestellung}

In dieser Erhebung sollte der Zusammenhang zwischen dem Angebot von Obst und Gemüse im Elternhaus bzw. der Mitnahme dieser Lebensmittel in die Schule und dem tatsächlichen 
Verzehr in Niedersachsen aktuell untersucht werden.

Darüber hinaus sollten die Auswirkungen des im Projekt „5 am Tag für Kids“ erworbenen Wissens über

Obst und Gemüse und die Empfehlungen für deren Verzehr auf den tatsächlichen Obst- und Gemüsekonsum analysiert werden. Diese Erkenntnisse sollen dazu dienen, das Konzept von „,5 am Tag für Kids“ wie auch andere wissensbasierte Interventionen zur Steigerung des Obst- und Gemüseverzehrs in ihrer Effektivität zu steigern.

\section{Methodik}

Im Rahmen der Projektevaluation nahmen 69 Klassen mit insgesamt 1376 Kindern, deren Eltern und 69 Lehrkräften an der Befragung teil.

Bei der Mehrheit der Klassen (65) handelte es sich um Grundschulen mit Dritt- und Viertklässlern.

Vier Klassen mit den Klassenstufen fünf bis sieben gehörten Förderschulen an. Das mittlere Alter der Kinder betrug 9,0 \pm 0,9 Jahre. $51 \%$ der Studienpopulation waren weiblich.

\section{Fragebögen}

Für die Datenerhebung wurden drei verschiedene Instrumente eingesetzt: Eltern- und Schülerfragebögen sowie ein Food Frequency Questionnaire zum Essverhalten der Schüler. Mit einem Elternfragebogen wurden soziodemografische Daten wie Haushaltsgröße, Geburtsländer der Eltern, Nationalität, Schulbildung und Berufsausbildung sowie Berufstätigkeit gewonnen. Darüber hinaus wurden die Eltern gefragt, ob sie ihrem Kind zu Hause täglich Obst, Gemüse bzw. Fruchtsaft zur Verfügung stellen, ob das Angebot von Obst und Gemüse variiert und ob sie ihrem Kind Obst und/oder Gemüse mit zur Schule geben. Die Eltern erhielten den Fragebogen, bevor ihr Kind am Projekt „,5 am Tag für Kids“ teilnahm. Mithilfe eines Schülerfragebogens wurden neben soziodemografischen Daten auch Angaben zu Alter, Größe und Gewicht gewonnen. Zusätzlich wurden die Kinder zu ihren Kenntnissen zum Thema Obst und Gemüse befragt. Die Schülerfragebögen wurden nach der Intervention ausgegeben. Das Essverhalten der Schülerinnen und Schüler wurde mit dem Food Frequency Questionnaire

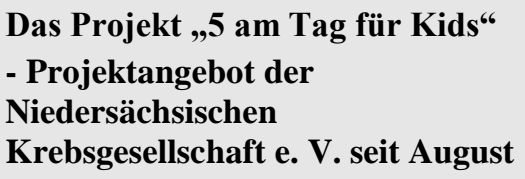

Das Projekt ,5 am Tag für Kids“

Projektangebot der

Krebsgesellschaft e. V. seit August

Ziel: Steigerung des Obst- und Gemüseverzehrs und Verbesserung der allgemeinen Essgewohnheiten

Zielgruppe: Dritt- und Viertklässler an niedersächsischen Grundschulen

Inhalt:

1. Theorieeinheit: Vermittlung von

Wissen über Vitamine, sekundäre

Portionsgrößen

Lebensmittelmarkt

3. Praxiseinheit: Zubereitung eines

kalten Gerichts 
(FFQ) „Was isst Ihr Kind?“ [16], der vom Robert-Koch-Institut beim Kinder- und Jugendgesundheitssurvey (KiGGS) eingesetzt wurde, zu drei Zeitpunkten ermittelt: vor (Baseline), ein (Follow-up 1) und drei Monate (Follow-up 2) nach der Intervention. Jeweils 1.376 Kinder bzw. deren Eltern erhielten den Fragebogen „Was isst Ihr Kind?“. Davon wurden 930 FFQs vor der Intervention ausgefüllt zurückgesendet. Zum Follow-up 1 waren dies 669 Fragebögen und beim Follow-up 2 noch 545 FFQs. Das ergibt Rücklaufquoten von $68 \%$, $49 \%$ und $40 \%$. Von 450 Schülerinnen und Schülern lagen alle FFQs der drei Zeitpunkte vor. Von 407 Studienteilnehmern wurden alle fünf Fragebögen (Eltern- und Schülerfragebogen, drei FFQs) vollständig zurückgegeben.

\section{Datenauswertung}

Die Auswertung der Daten erfolgte mithilfe der Statistik-Software SPSS (Version 20.0). Der Kolmogorov-Smirnov-Test wurde zur Überprüfung der Normalverteilung herangezogen. Unterschiede zwischen den Gruppen wurden mit dem Mann-Whitney-U-Test für kontinuierliche Variablen auf statistische Signifikanz geprüft. Zusammenhänge zwischen den Ziel- und Einflussvariablen wurden mittels einer binären logistischen Regressionsanalyse beschrieben. Diese Regressionsanalyse wurde mit der Statistik-Software SPSS (Version 21.0) durchgeführt. Für alle Analysen wurde ein Signifikanzniveau von $\mathrm{p} \leq 0,05$ angenommen.

\section{Ergebnisse}

Eine signifikante Zunahme des Obst- und Gemüseverzehrs konnte im Verlauf der Messungen mithilfe der FFQs über den Zeitraum von drei Monaten nicht verzeichnet werden. Für die nachfolgende Analyse wurden die Daten der FFQs herangezogen, die das Ernährungsverhalten der Kinder vor der Intervention erfassten. Hier konnten wichtige Einflussfaktoren auf den kindlichen Obst- und Gemüseverzehr ermittelt werden. Die Unterschiede im Verzehr von Obst, Gemüse und Saft sowie dessen Gesamtmenge zwischen Schülerinnen und Schülern, die im Elternhaus diese Lebensmittel zur Verfügung gestellt bekommen bzw. die Obst und Gemüse mit zur Schule nehmen und denen, die diese Nahrungsmittel nicht angeboten bekommen, werden in - Tabelle 2 deutlich. 
Tabelle 2: Obst-, Gemüse- und Saftverzehr in g/Tag in Abhängigkeit vom Angebot im Elternhaus und in der Schule

\begin{tabular}{|c|c|c|c|c|c|c|c|c|c|}
\hline & \multicolumn{2}{|c|}{ Obst zu Hause } & \multirow[t]{2}{*}{$\mathbf{p}$} & \multicolumn{2}{|c|}{$\begin{array}{l}\text { Gemüse zu } \\
\text { Hause }\end{array}$} & \multirow[t]{2}{*}{$\mathbf{p}$} & \multicolumn{2}{|c|}{$\begin{array}{c}\text { Obst/Gemüse in } \\
\text { Schule }\end{array}$} & \multirow[t]{2}{*}{$\mathbf{p}$} \\
\hline & $\begin{array}{c}\text { ja } \\
\mathrm{n}=765\end{array}$ & $\begin{array}{c}\text { nein } \\
n=60\end{array}$ & & $\begin{array}{c}\mathrm{ja} \\
\mathrm{n}=674\end{array}$ & $\begin{array}{c}\text { nein } \\
n=149\end{array}$ & & $\begin{array}{c}\mathrm{ja} \\
\mathrm{n}=597\end{array}$ & $\begin{array}{c}\text { nein } \\
\mathrm{n}=230\end{array}$ & \\
\hline Obst $^{1}$, g/Tag & $\begin{array}{l}261 \\
\pm 233\end{array}$ & $\begin{array}{l}86 \\
\pm 109\end{array}$ & $* * *$ & $\begin{array}{c}263 \\
\pm 237\end{array}$ & $\begin{array}{c}183 \\
\pm 187\end{array}$ & $* * *$ & $\begin{array}{l}286 \\
\pm 237\end{array}$ & $\begin{array}{l}150 \\
\pm 181\end{array}$ & $* * *$ \\
\hline Gemüse $^{2}$, g/Tag & $\begin{array}{l}151 \\
\pm 125\end{array}$ & $\begin{array}{l}100 \\
\pm 106\end{array}$ & $* * *$ & $\begin{array}{c}161 \\
\pm 128\end{array}$ & $\begin{array}{c}82 \\
\pm 79\end{array}$ & $* * *$ & $\begin{array}{l}160 \\
\pm 124\end{array}$ & $\begin{array}{l}116 \\
\pm 119\end{array}$ & $* * *$ \\
\hline Fruchtsaft $^{3}$, g/Tag & $\begin{array}{l}273 \\
\pm 363\end{array}$ & $\begin{array}{l}105 \\
\pm 162\end{array}$ & $* * *$ & $\begin{array}{c}265 \\
\pm 354\end{array}$ & $\begin{array}{c}240 \\
\pm 356\end{array}$ & ns & $\begin{array}{l}277 \\
\pm 366\end{array}$ & $\begin{array}{l}220 \\
\pm 323\end{array}$ & $* * *$ \\
\hline $\begin{array}{l}\text { Gesamtmenge } \\
\text { Obst/Gemüse/Saft, } \\
\text { g/Tag }\end{array}$ & $\begin{array}{l}686 \\
\pm 486\end{array}$ & $\begin{array}{l}291 \\
\pm 222\end{array}$ & $* * *$ & $\begin{array}{c}689 \\
\pm 484\end{array}$ & $\begin{array}{c}506 \\
\pm 448\end{array}$ & $* * *$ & $\begin{array}{l}722 \\
\pm 488\end{array}$ & $\begin{array}{l}487 \\
\pm 425\end{array}$ & $* * *$ \\
\hline
\end{tabular}

${ }^{1}$ frisches Obst, gekochtes Obst und Konservenobst

${ }^{2}$ gekochtes Gemüse, TK-Gemüse, Konservengemüse, Blattsalate, Rohkost und rohes Gemüse

${ }^{3}$ Fruchtsaft, Fruchtnektar und Gemüsesaft (auch verdünnt)

Alle Werte sind Mittelwert \pm Standardabweichung

ns $=$ nicht signifikant

$* \mathrm{p} \leq .05$

$* * \mathrm{p} \leq .01$

$* * * \mathrm{p} \leq .001$

${ }^{a}$ berechnet mit dem Mann-Whitney-U-Test

Kinder, die über den Zugang zu Obst und Gemüse verfügten, aßen signifikant und relevant mehr davon. Darüber hinaus wurde der Zusammenhang analysiert zwischen dem täglichen Verzehr von mindestens einer Portion Obst bzw. einer Portion Gemüse (laut FFQ) und den Faktoren: - „Wissen“ der Kinder (persönlicher Faktor, Schülerbefragung nach der Intervention) - physische Verfügbarkeit von Obst und Gemüse im Elternhaus (Elternfragebogen vor der Intervention) und - „Mitgabe in die Schule“ (sozialer Umweltfaktor, Elternfragebogen vor der Intervention) (• Tabelle 3$)$.

Tabelle 3: Zusammenhang zwischen täglichem Obst-/Gemüseverzehr und Einflussfaktoren darauf

Odds Ratio und Konfidenzintervall einer binären logistischen Regressionsanalyse, n=706

\begin{tabular}{|l|l|l|}
\hline persönliche und Umweltfaktoren & $\begin{array}{l}\text { mindest. 1 Portion Obst } \\
\text { täglich }\end{array}$ & $\begin{array}{l}\text { mindest. 1 Portion Gemüse } \\
\text { täglich }\end{array}$ \\
\hline OR $(\mathbf{9 8} \% \mathbf{~ K I})$ & OR (98 \% KI) \\
\hline Wissen der Kinder & $0,76(0,35-1,65)$ & $0,99(0,50-1,95)$ \\
\hline „Mitgabe in die Schule“ & $\underline{4,15}(2,70-6,36)$ & $\underline{1,70}(1,13-2,55)$ \\
\hline $\begin{array}{l}\text { Obst zu Hause } \\
\text { Gemüise zu Hause }\end{array}$ & $\underline{6,99}(2,91-16,76)$ & $\underline{1,82}(0,81-4,06)$ \\
\hline $\begin{array}{l}\text { OR = Odds Ratio; KI = Konfidenzintervall } \\
\text { signifikante OR sind unterstrichen }\end{array}$ & $\underline{1,69}(1,00-2,86)$ & \\
\hline
\end{tabular}


Statistisch signifikante Ergebnisse konnten bezogen auf die physische Verfügbarkeit (Obst/Gemüse zu Hause) und den Faktor „Mitgabe in die Schule“ festgestellt werden. Weiterhin wurden auch die Zusammenhänge zwischen dem Verzehr von mindestens einer Portion Obst bzw. Gemüse und Faktoren wie Migrationshintergrund der Eltern, Stellenwert der Ernährung bei den Kindern und vorhandene Lebensmittelallergien überprüft. Hier fanden sich keine signifikanten Korrelationen (Daten nicht gezeigt).

\section{Diskussion}

Die Ergebnisse der vorliegenden Untersuchung belegen, dass insbesondere soziale Faktoren und die damit assoziierte Verfügbarkeit eine wichtige Rolle für den Obst- und Gemüseverzehr der Kinder spielen. Das tägliche Anbieten von Obst und Gemüse im Elternhaus, aber auch in Form eines Pausenfrühstücks in der Schule, stellt einen entscheidenden Faktor dar, die Kinder zum Verzehr zu ermuntern. Ergebnisse anderer Studien legten darüber hinaus nahe, dass nicht nur das generelle Vorhandensein dieser Lebensmittel im Lebensumfeld, sondern auch die Darreichungsform von Bedeutung ist. Steht Kindern das Obst oder Gemüse in verzehrfertiger Form z.B. in Form von kleingeschnittenen Stücken oder Sticks zur Verfügung, greifen sie vermehrt zu [17]. Es gibt zudem Hinweise darauf, dass die kognitive Vermittlung von Informationen über den gesundheitlichen Nutzen von Obst und Gemüse und den entsprechenden Verzehrempfehlungen ebenfalls positiv mit dem Verzehr assoziiert sind [17]. Diesen Zusammenhang konnten die Ergebnisse dieser Studie jedoch nicht untermauern. Die rein wissensbasierte Intervention „5 am Tag für Kids“ hatte nicht den gewünschten Effekt einer Verhaltensänderung. Die Einführung von Verhältnispräventionsmaßnahmen wie z.B. (kostenlose) Schulobst-Programme gelten ebenfalls als erfolgversprechender Prädiktor zur Förderung des Obst- und Gemüseverzehrs von Schülerinnen und Schülern. In der vorliegenden Untersuchung konnte gezeigt werden, dass Schülerinnen und Schüler, die durch ihre Eltern Obst und Gemüse für die Pausenverpflegung in der Schule erhalten, auch tatsächlich mehr von diesen Lebensmitteln zu sich nehmen. Die institutionelle Bereitstellung von Obst und/oder Gemüse hat in anderen europäischen Ländern (nachhaltige) signifikante Effekte gezeigt [1820]. In Deutschland nahmen bislang nur sieben Bundesländer am EU-Schulobstprogramm teil. Die verpflichtende Evaluation für das Schuljahr 2010-2011 ergab in fast allen teilnehmenden Bundesländern einen erhöhten Verzehr von Obst und Gemüse. Allerdings ist in diesem Zusammenhang anzumerken, dass die Verzehrdaten oft auf Einschätzung durch Eltern oder Lehrkräfte beruhen [21]. Trotz dieser methodischen Problematik erscheint eine Erweiterung 
des institutionellen Angebots von Obst und Gemüse als eine sinnvolle Maßnahme zur Steigerung des Verzehrs dieser Lebensmittel. Aufgrund der klaren Erkenntnis, dass Eltern einen erheblichen Einfluss auf die Verzehrgewohnheiten ihrer Kinder - und damit auch deren Obstund Gemüsekonsum - haben, sollte bei der Konzeptionierung von schulischen Interventionen darauf geachtet werden, Eltern soweit irgend möglich partizipativ einzubinden.

\section{Limitationen}

Um die Zusammenhänge zwischen dem Verzehr von Obst und Gemüse und den Einflussfaktoren konkreter beschreiben/bestimmen zu können, hätten die Angaben im Elternfragebogen detaillierter formuliert sein müssen, z.B. welche Obst- und Gemüsesorten im Elternhaus vorhanden sind, wie sie den Kindern zugänglich gemacht werden und ob sie den Kindern verzehrfertig angeboten werden. Darüber hinaus hätten in dem Elternfragebogen auch Daten zum Verzehrverhalten der Eltern erhoben werden können, um auch diesbezügliche Korrelationen erfassen zu können. Der Schwerpunkt der Evaluation war die Erfassung der kurzund mittelfristigen Effekte des Projekts „5 am Tag für Kids“ auf den realen Verzehr. Daher stand das Erhebungsinstrument FFQ im Mittelpunkt. Das mehrmalige Ausfüllen war für die Eltern bereits mit einem größeren Aufwand verbunden. Die geringe Rücklaufquote ist ein Indiz dafür, dass die Eltern die erforderliche Zeit zur Beantwortung der Erhebungsbögen nicht aufwenden konnten oder wollten. Zusätzliche umfangreichere bzw. detailliertere Fragebögen hätten daher evtl. eine noch geringere Responserate mit sich gebracht. Die Ergebnisse lassen weiterhin vermuten, dass ein gewisser Selektionseffekt eingetreten ist und vorrangig diejenigen Eltern sämtliche Erhebungsinstrumente ausgefüllt haben, die ein besonderes Interesse an einer gesunden Ernährung ihres Kindes/ihrer Kinder haben, was sich im hohen Obst- und Gemüseverzehr der Kinder widerspiegelt. Auch ist eine Beantwortung gemäß sozialer Erwünschtheit als Ursache für den deutlich höheren Durchschnittsverzehr von Obst und Gemüse im Vergleich zu KiGGS und EsKiMo (Ernährungsstudie als KiGGS-Modul) nicht auszuschließen. 


\section{Literatur}

1. Hempel U. Erste Ergebnisse der KiGGS-Studie zur Gesundheit von Kindern und Jugendlichen in Deutschland. Berlin: Robert Koch-Institut (2006) URL: www.rki. de/DE/Content/Gesundheitsmonitoring/Studien/Kiggs/Basiserhebung/Ergebnisbroschüre.pdf?_blob=publicationFile Zugriff 18.02.2013.

2. Mensink GBM, Heseker H, Richter A, Stahl A, Vohmann C. Forschungsbericht. Ernährungsstudie als KiGGS-Modul (EsKiMo). Bonn: Robert-Koch-Institut/Universität Paderborn (2007).

3. Heseker H, Mensink GBM Lebensmittelverzehr und Nährstoffzufuhr im Kindes-und Jugendalter - Ergebnisse aus den beiden bundesweit durchgeführten Ernährungsstudien VELS und EsKiMo. In: Ernährungsbericht 2008. Deutsche Gesellschaft für Ernährung e. V., Bonn (2008).

4. Brug J, Tak NI, te Velde SJ et al. (2008) Taste preferences, liking and other factors related to fruit and vegetable intakes among schoolchildren: results from observational studies. Br J Nutr 99 Suppl 1: S7-S14.

5. De Bourdeaudhuij I, te Velde S, Brug J et al. (2008) Personal, social and environmental predictors of daily fruit and vegetable intake in 11-year-old children in nine European countries. Eur J Clin Nutr 62: 834-841.

6. Tak NI, te Velde SJ, Brug J (2008) Are positive changes in potential determinants associated with increased fruit and vegetable intakes among primary schoolchildren? Results of two intervention studies in the Netherlands: The Schoolgruiten Project and the Pro Children Study. Int J Behav Nutr Phys Act 5: 21.

7. Patrick H, Nicklas TA (2005) A review of family and social determinants of childrens' eating patterns and diet quality. Journal of the American College of Nutrition 24 (2): 83-92.

8. Rasmussen M, Krølner R, Klepp K-I et al. (2006) Determinants of fruit and vegetable consumption among children and adolescents: a review of the literature. Part I: quantitative studies. Int J Behav Nutr Phys Act 3: 22.

9. Bjarnason B. Untersuchung von Präferenzen für Obst und Gemüse von Grundschulkindern - Befragungen in fünf Schulklassen im Landkreis Rastatt - Justus-Liebig-Universität Gießen, [Dissertation], Gießen (2011). 
10. Krølner R, Rasmussen M, Brug J et al. (2011) Determinants of fruit and vegetable consumption among children and adolescents: a review of the literature. Part II: qualitative studies. Int J Behav Nutr Phys Act 8: 112.

11. Baxter IA, Schröder MJA (1997) Vegetable consumption among Scottish children: a review of the determinants and proposed strategies to overcome low consumption. Brit Food J: $380-387$.

12. Philipps U. Evaluation gesundheitsfördernder Maßnahmen bezüglich des Ernährungsverhaltens von Grundschulkindern, Verlag Julius Klinkhardt, [Dissertation], Bad Heilbrunn (2004).

13. De Sa J, Lock K (2007) School-based fruit and vegetable schemes: a review of the evidence. London School of Hygiene and Tropical Medicine. URL: http://ec.europa.eu/agriculture/sfs/documents/de_sa_lock_en.pdf Zugriff 08.09.2015.

14. Molderings M. Evaluation pädagogisch-didaktischer Ansätze im Rahmen der gesundheitsförderlichen Ernährungserziehung der Grundschule: Einfluss von Unterrichtsformen, Elterneinbeziehung und Zielvereinbarung hinsichtlich einer Veränderung des Ernährungsverhaltens. Schneider Verlag Hohengehren, [Dissertation], Baltmannsweiler (2007).

15. Klepp KI, Pérez-Rodrigo C, De Bourdeaudhuij I et al. (2005) Promoting fruit and vegetable consumption among European schoolchildren: rationale, conceptualization and design of the Pro Children Project. Annals of Nutrition Metabolism 49: 212-220.

16. Mensink GBM, Burger M (2004) Was isst du? Ein Verzehrshäufigkeitsfragebogen für Kinder und Jugendliche. Bundesgesundheitsblatt - Gesundheitsforschung - Gesundheitsschutz 47: 219-226.

17. Blanchette L, Brug J (2005) Determinants of fruit and vegetable consumption among 612-year-old children and effective interventions to increase consumption. J Hum Nutr Dietet 18: 431-443.

18. Bere E, Veierød MB, Bjelland M et al. (2006) Free school fruit - sustained effect 1 year later. Health Educ Res 21 (2): 268-275.

19. Bere E, Veierød MB, Skare $\varnothing$ et al. (2007) Free school fruit - sustained effect three years later. Int J Behav Nutr Phys Act 4: 5.

20. Fogarty AW, Antoniak M, Venn AJ et al. (2007) Does participation in a populationbased dietary intervention scheme have a lasting impact on fruit intake in young children? Int J Epidemiol 36: 1080-1085. 
21. Bundesministerium für Ernährung, Landwirtschaft und Verbraucherschutz. Zusammenfassung der Evaluationsergebnisse der in der Bundesrepublik Deutschland am EU-Schulobstprogramm teilnehmenden Länder für das Schuljahr 2010-2011 URL: www.bmelv.de/SharedDocs/Downloads/ Ernaehrung/Kita-Schule/EU-Schulfrucht_ Evaluationsbericht.pdf?_blob=publica tionFile Zugriff 17.06.2013. 
Praktische Ernährungsbildung in der 5./6. Klasse: Evaluation des Projekts „Entdeckungsreise Essen“

Praktische Ernährungsbildung in der 5./6. Klasse: Evaluation des Projekts „Entdeckungsreise Essen“

Autoren: Helen Schöberle, Nadine Wagner, Silke Lichtenstein, Catrin Ranke,

Silke Mittmann, Anja Austel ${ }^{\dagger}$, Thomas Ellrott

Eingereicht bei Ernährungs Umschau. 


\section{Essen“}

\section{Einführender Absatz}

Gesellschaftliche Veränderungen führen dazu, dass umfassende Ernährungskompetenz1 immer seltener im familiären Kontext vermittelt wird. Sie gilt jedoch als Schlüsselfaktor für ein gesundheitsförderndes Essverhalten. Sekundäre Sozialisationsinstanzen sind daher aufgefordert, diese Defizite zu kompensieren. Neue Konzepte der schulischen Ernährungsbildung fördern praktische Kompetenzen und haben einen klaren Alltagsbezug. Allerdings erschweren limitierte Ressourcen an Schulen oft die Umsetzung solcher Konzepte. Das Projekt „Entdeckungsreise Essen“ (EE) lässt aufgrund seiner Modulstruktur eine flexible Anpassung an die Ressourcen der Schulen zu. Bewertung und Wirksamkeit der Module wurden in einer Pilotstudie überprüft.

Schlüsselwörter: Ernährungsbildung, Essverhalten, Ernährungskompetenz, explorativer Ansatz, Evaluation

\footnotetext{
${ }^{1}$ „Ernährungskompetenz wird als die Fähigkeit definiert, theoretische Kenntnisse und praktische Fertigkeiten im Ernährungsalltag in ein angemessenes Handeln umzusetzen. Ernährungskompetenz als eine Schlüsselkompetenz in der Daseinsvorsorge unterstützt die Bewältigung und Gestaltung des Alltagslebens sowohl im häuslichen Ernährungsalltag (Koch- und Konsumkompetenzen) als auch im Marktgeschehen (u. a. Lebensmitteleinkauf). " [1]
} 


\section{Einleitung und Hintergrund der Studie}

Sowohl die Verfügbarkeit von hochwertigen Lebensmitteln, als auch das Wissen um eine gesunde Ernährungsweise sind in modernen westlichen Gesellschaften besser als je zuvor [2], was sich folglich auch in einer stetigen Verbesserung der Ernährungsgewohnheiten widerspiegeln müsste. Trotz eines zunehmenden Wunsches nach gesunder Ernährung besteht eine Diskrepanz zwischen einem ansteigenden theoretischen Ernährungswissen darüber und dessen praktischer Umsetzung im alläglichen Leben.

In Deutschland ist die Prävalenz von Fehl-, Unter- und Überernährung im Kindesalter auf einem mittelhohem Niveau und nimmt teilweise weiter $\mathrm{zu}[3 ; 4]$. Gerade die kindliche Entwicklungsphase spielt bei der Prävention von Ernährungsproblemen im Erwachsenenalter eine maßgebliche Rolle, da in dieser Phase erworbene Ernährungsgewohnheiten meist das ganze Leben bestehen bleiben und langfristig die Gesundheit beeinflussen [5; 6]. Ebenso wie Gesundheit die Voraussetzung für Bildung darstellt, ist Bildung eine grundlegende Voraussetzung für Gesundheit [7].

Die Relevanz schulischer Ernährungsbildung ist heute höher als in zurückliegenden Jahrzehnten [8]. Infolge einer zunehmenden Berufstätigkeit beider Elternteile, veränderter Arbeitszeiten und der Abkehr vom klassischen Kernfamilien-Modell kommt es zu einer Umverteilung familiärer Aufgaben [9]. Kinder verbringen heute viel mehr Zeit in Kindertageseinrichtungen und Schulen. Diesen Betreuungs- und Bildungseinrichtungen kommt damit ein höherer Stellenwert für eine gesundheitsfördernde Ernährungserziehung und Ernährungsbildung zu. Als sogenannter „Setting-Ansatz“ bieten diese Lernorte Vorteile, weil aufgrund der allgemeinen Schulpflicht alle Kinder und Jugendliche erreicht werden können [10; $11]$.

Seit langem stellt sich die Frage, was den Transfer von theoretischem Ernährungswissen in entsprechendes praktisches Handeln hemmt [12] und welche kindlichen Kompetenzen gefördert werden müssen, damit ein Übertrag gelingt [13]. Die neueren Ansätze der Ernährungsbildung fokussieren in Anlehnung an das Konzept der Gesundheitsförderung (Salutogenese) auf Faktoren, die Individuen widerstandsfähig gegen Stressoren machen [14; 15]. Kinder, Jugendliche und Erwachsene sollen dazu befähigt werden, Informationen und Erfahrungen zu reflektieren, um Alltagssituationen auch unter sozialen, ökologischen und ökonomischen Aspekten selbständig und gesundheitsfördernd gestalten zu können. Gemäß des aktuellen Verständnisses von Ernährungsbildung erfordert dies v. a. individuelle 
handlungsorientierte Ansätze, die einen klaren Alltagsbezug aufweisen und Inhalte über praktische Fertigkeiten (Handeln lernen) vermitteln [12; 13].

Derzeit existieren verschiedene daraus abgeleitete Konzepte zur Ernährungsbildung für unterschiedliche Altersgruppen. Unter dem Titel „aid macht Schule“, ,aid Ernährungsführerschein“ und „SchmExperten“, gibt der „Auswertungs- und Informationsdienst Ernährung, Landwirtschaft, Verbraucherschutz e. V.“ (aid) Informationen und Arbeitshilfen für Schulen heraus, welche in der Grundschule bzw. weiterführenden Schulen eingesetzt werden können [16; 17]. Alle Materialien basieren auf dem evaluierten „Reform der Ernährungs- und Verbraucherbildung an Schulen“-Curriculum (REVIS), welches praktische Ernährungsbildung in Form der Förderung von Handlungskompetenzen mit der Ausrichtung auf die Lebenswelt der Kinder und Jugendlichen in den Vordergrund stellt [18]. Trotz der belegten Effektivität der Ernährungsbildung nach REVIS, sind die Elemente bundesweit (noch) nicht Bestandteile des Regel-Lehrplanes. Als einziges Bundesland hat Schleswig-Holstein ein Unterrichtsfach zur Verbraucherbildung für die Sek. I der allgemeinbildenden Schulen (Klasse 5-10) zum Schuljahr 2009/2010 eingeführt [19].

Mögliche Ursachen für die fehlende Integration zeitgemäßer Ernährungsbildungskonzepte in Schulen können begrenzte finanzielle, personelle und/oder räumliche Ressourcen von Schulen sein. Den meisten Schulen steht zudem nur begrenzt Zeit zur Verfügung, um zusätzliche Konzepte und Projekte jenseits des Lehrplans umzusetzen. Ernährungsbildung muss daher strukturelle Kriterien der Umsetzbarkeit für Schulen erfüllen [20].

\section{Das Konzept „Entdeckungsreise Essen“}

Das praktische Ernährungsbildungskonzept „Entdeckungsreise Essen“(EE) wurde vom Institut für Ernährungspsychologie an der Universitätsmedizin Göttingen in Kooperation mit Praxispartnern ${ }^{2}$ entwickelt und fokussiert auf die Umsetzbarkeit unter den oben beschriebenen Rahmenbedingungen an Schulen.

Das Projekt richtet sich an Schüler der 5. und 6. Klasse. Wesentliche Bestandteile des pädagogischen Konzepts sind das Lernen vom Modell (Beobachtungslernen) und das Lernen durch positive Verstärkung. Als praxisnahes Programm liegt der Fokus auf der Förderung von Handlungskompetenzen rund um Essen und Trinken, auf der Mahlzeitenzubereitung sowie auf

\footnotetext{
${ }^{2}$ Bürgerstiftung Göttingen, Vogt-Realschule (ehemals Personn-Realschule Göttingen-Weende), Club der Köche Göttingen und Verbraucherzentrale Göttingen
} 
der Erfahrung von Esskultur und Lebensmittelvielfalt. Charakteristisch für das Projekt ist der teilhabende und explorierende Ansatz. Gemeinsames Entdecken, Schmecken und Selbermachen, fernab von starren Lernzielen und Benotung ist das Ziel [21]. Dies fördert individuell vorhandene Kenntnisse und Fertigkeiten und erlaubt es, erworbene Kompetenzen auf alltägliche Ess-Situationen selbstständig zu übertragen [11]. Das gemeinsame Zubereiten und der Genuss mit allen Sinnen stärkt darüber hinaus die Wertschätzung für Lebensmittel und das Vertrauen in die eigenen Fertigkeiten (Selbstwirksamkeit) [22].

Entsprechend der Vorgaben des Niedersächsischen Kultusministeriums für didaktische Methoden wird in besonderer Art und Weise die Verknüpfung von Theorie und Praxis angestrebt, um so ein größtmögliches Lernergebnis zu erzielen [23]. Bei EE wird dies durch die Kombination aus praktischen Kocheinheiten („Erlebniskochen“) und praxisnahen partizipativen „Entdeckermodulen“ gewährleistet.

Das „Erlebniskochen“ in der Lehrküche der Schule wird im Idealfall von professionellen lokalen Köchen (z.B. aus Restaurants, Betriebskantinen, Mensen) durchgeführt und von Lehrern begleitet. Die Schüler lernen beim gemeinsamen Kochen und Essen direkt umsetzbare Handlungskompetenzen. Köche und Lehrer wirken dabei als positiv besetzte Vorbilder. Es gibt aber auch einen Lernprozess der Schüler voneinander (Beobachtungslernen). Das erfolgreiche Kochen und geschätzte gemeinsame Essen ergänzt den Lernprozess durch operantes Konditionieren.

Die „Entdeckermodule“ hingegen können von Lehrern selbst im Klassenraum umgesetzt werden. Sie setzen keine besondere räumliche Ausstattung voraus. Auch hier wird der Lernerfolg durch eine angenehme Arbeitsatmosphäre, Freude/Spaß, Erfolg und Genuss positiv verstärkt [21]. Die Durchführung der „Entdeckermodule“ durch eigene Lehrer fördert die Identifizierung des Kollegiums mit moderner Ernährungsbildung. Dabei eröffnen die Module einen vergleichsweise großen Spielraum für die konkrete Umsetzung in der Praxis. Die Gesundheitssystemforschung belegt, dass die Möglichkeit einer Re-Invention durch die Akteure selbst einen Erfolgsfaktor bei der Verstetigung von Projekten darstellt. Eine Verstetigung ist umso wahrscheinlicher, je größer der Gestaltungsspielraum der Akteure (hier: der Lehrer und Schüler) ist [24]. Auch dies wurde bei der konzeptionellen Entwicklung berücksichtigt.

Im Hinblick auf die teilweise eingeschränkten Möglichkeiten der Schulen bezüglich der Ausstattung sind die „Entdeckermodule“ im Projekt EE so gestaltet, dass keine speziellen Lehrküchen nötig sind, sondern normale Klassenräume genutzt werden können. Tabelle 1 zeigt 
Inhalte und Praxisbezug der jeweils 1,5 stündigen im Klassenraum durchführbaren „Entdeckermodule“ im Überblick.

Tabelle 1: Übersicht Entdeckermodule (Aktionslevel: + niedrig, ++ hoch, +++ sehr hoch)

*Unter Aktionslevel wird das Ausmaß der praktischen Mitarbeit der Kinder verstanden.

\begin{tabular}{|c|c|c|}
\hline $\begin{array}{l}\text { Modul } \\
\text { (Aktionslevel*) }\end{array}$ & Inhalte & Praxis \\
\hline $\begin{array}{l}\text { Lebensmittelquiz } \\
(+)\end{array}$ & $\begin{array}{l}\text { Ratespiel mit Lebensmittelfoto- } \\
\text { Puzzles, } \\
\text { sukzessives Aufdecken }\end{array}$ & $\begin{array}{l}\text { Nach dem Ratespiel: Riechen, } \\
\text { Tasten, Schmecken der zu } \\
\text { erratenden Lebensmittel }\end{array}$ \\
\hline $\begin{array}{l}\text { Sandwichwettbewerb } \\
(+++)\end{array}$ & $\begin{array}{l}\text { Rezeptwettbewerb, } \\
\text { Ergebnisbewertung nach } \\
\text { Attraktivität }\end{array}$ & $\begin{array}{l}\text { Zubereitung, Kreativität, } \\
\text { sinnliches Erfahren }\end{array}$ \\
\hline $\begin{array}{l}\text { Lassi, Smoothie und } \\
\text { Co. } \\
(+++)\end{array}$ & $\begin{array}{l}\text { Kennenlernen attraktiver } \\
\text { Mixgetränke }\end{array}$ & $\begin{array}{l}\text { Erraten, Verkosten und } \\
\text { Zubereiten von Obst und Milch } \\
\text { in neuen Zubereitungsformen }\end{array}$ \\
\hline $\begin{array}{l}\text { Ran an die Kartoffel } \\
(++)\end{array}$ & $\begin{array}{l}\text { Exkurs in die Esskultur am Beispiel } \\
\text { Kartoffel }\end{array}$ & $\begin{array}{l}\text { Kennenlernen verschiedener } \\
\text { Sorten, Zubereitung }\end{array}$ \\
\hline $\begin{array}{l}\text { Im Reich der Sinne } \\
(++)\end{array}$ & $\begin{array}{l}\text { Kennenlernen, Erraten, } \\
\text { Wissenswertes zu Gewürzen }\end{array}$ & $\begin{array}{l}\text { Sinnliches Erfahren von } \\
\text { Gewürzen }\end{array}$ \\
\hline $\begin{array}{l}\text { Exkursion: } \\
\text { Käsehof } \\
(+++)\end{array}$ & $\begin{array}{l}\text { Besuch eines regionalen Käsehofes, } \\
\text { Besichtigung, Herstellungsprozess }\end{array}$ & $\begin{array}{l}\text { Käse selber machen als } \\
\text { Mitbringsel für zu Hause }\end{array}$ \\
\hline
\end{tabular}

In den Modulen wird auf eine Wertung von Lebensmitteln in gut/schlecht bzw. gesund/ungesund komplett verzichtet, da entsprechendes Wissen das Ernährungsverhalten von Kindern nicht oder sogar gegenteilig beeinflusst [25]. Zudem kann eine solche Wertung - wenn überhaupt - nur für die Gesamtauswahl über einen längeren Zeitraum gemacht werden, nicht für ein einzelnes Lebensmittel, das nur eine vergleichsweise kleine Teilqualität der Gesamternährung darstellt [26]. 


\section{Evaluation des Einsatzes ausgewählter Module im Unterricht}

In einer Pilotstudie wurden drei der vorbeschriebenen Entdeckermodule im Schuleinsatz überprüft [27]. Im Mittelpunkt stand die Forschungsfrage, ob so die Ernährungskompetenz der Schüler verbessert werden kann und ob eine Transmission in den außerschulischen Alltag stattfindet.

Untersucht wurden hierzu drei Hypothesen [27]:

1. Die Zielgruppe kann über die Entdeckermodule erreicht werden.

2. Je höher der Anteil teilhabender und praktischer Elemente ist, desto besser bewertet die Zielgruppe das entsprechende Entdeckermodul.

3. Über die Entdeckermodule können neue Kompetenzen in den Ernährungsalltag der Zielgruppe Einzug halten (Transmission).

\section{Methoden}

Für die Evaluation wurde eine Schule ausgewählt, die nicht an der Konzeptentwicklung beteiligt war ${ }^{3}$. Die Untersuchung basierte auf einem Zweigruppen-Pretest-Posttest-Design. Alle Schüler ( $n=82)$ wurden je nach Teilnahmebereitschaft der Hauswirtschaftslehrer, entweder der Interventionsgruppe (IG) oder der Kontrollgruppe (KG) zugeordnet (Tabelle 2). Während die IG im Verlauf der dreiwöchigen Untersuchung die Module durchführte, erhielt die KG Hauswirtschaftsunterricht nach Lehrplan. Eine Kontrollgruppe ganz ohne parallele Intervention war wegen des obligatorischen Hauswirtschaftsunterrichts in der 6. Jahrgangsstufe in dieser Schule nicht möglich. Für die Evaluation wurden drei exemplarische Module ausgewählt: „Lebensmittelquiz“, „Lassi, Smoothie und Co.“" sowie "Sandwichwettbewerb“ (vgl. Tab. 1). Die Durchführung der Module oblag Hauswirtschaftslehrern auf Basis eines standardisierten Manuals [27].

Zur Evaluation wurden drei Erhebungsinstrumente eingesetzt:

- Schriftliche Befragungen mittels standardisierter Fragebögen (T0/vor Intervention; T3/nach Intervention)

- Passiv-teilnehmende Beobachtungen mittels unstrukturierter Beobachtungsbögen (T1)

- „Ein-Punkt-Abfrage“ mittels Bewertungsplakaten (T2)

\footnotetext{
${ }^{3}$ Integrierte Gesamtschule (IGS) Mühlenberg Hannover, 6. Jahrgangsstufe ,Schuljahr 2010/2011
} 
Die zeitliche Abfolge der Datenerhebung wird in Abbildung 1 dargestellt.

Abbildung 1: Studiendesign (I=Intervention, T1-3= Erhebungszeitpunkte) [27]

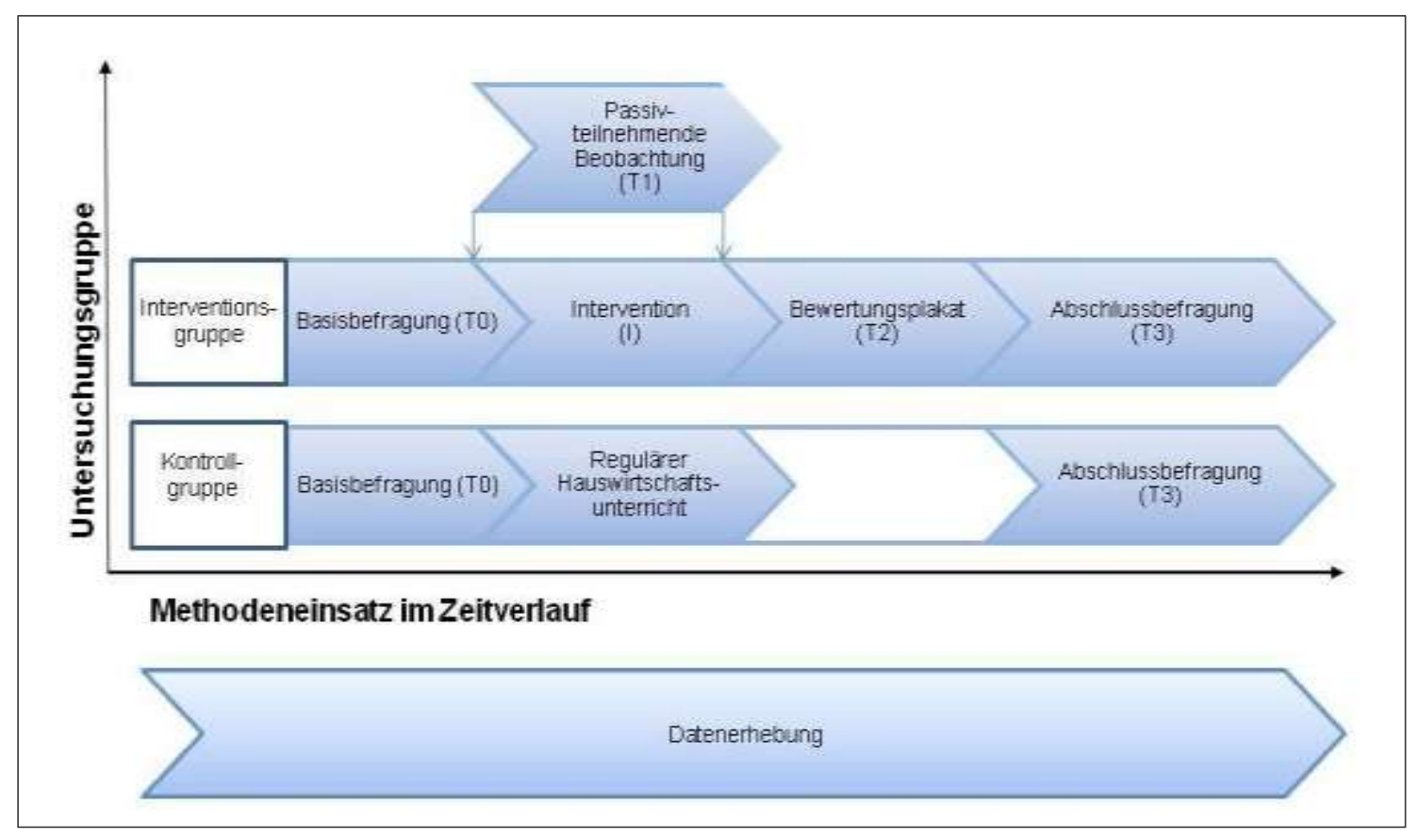

Nachfolgend werden die Ergebnisse der schriftlichen Befragung (T0; T3) dargestellt. Die Ergebnisse der Passiv-teilnehmenden Beobachtung (T1) sowie die der „Ein-Punkt-Abfrage“ (T2) unterstützen die Erkenntnisse aus den schriftlichen Befragungen [27].

Vor der Interventionsphase wurde in beiden Gruppen mittels eines identischen Eingangsfragebogens nach familiären Ernährungsgewohnheiten z.B. bezüglich Mahlzeitenzubereitung oder Lebensmitteleinkauf gefragt. Zusätzlich wurden basale soziodemographische Daten erhoben. Nach der Intervention erhielten die Gruppen zwei unterschiedliche Abschlussfragebögen. Die Version für die IG beinhaltete u. a. Fragen zur subjektiven Beurteilung der Module anhand von Schulnoten. Um eine etwaige Transmission der Lerninhalte auf das außerschulische Lebensumfeld zu erfassen, wurden die eingangs gestellten Fragen zu häuslichen Ernährungsgewohnheiten erneut gestellt. Die Version für die KG war nahezu identisch zum Eingangsfragebogen konzipiert. Die Auswertung der Daten erfolgte anonymisiert [27]. 


\section{Ergebnisse}

\section{Stichprobe}

Das ausgewertete Gesamtkollektiv umfasste 82 Schüler aus sieben Kursen. Vier Kurse bildeten die IG (n=49) und drei Kurse die KG (n=33). Das Alter der Schüler (Gesamtstichprobe) lag zum Zeitpunkt der Befragung zwischen 11 und 13 Jahren (MW=11,4; SD=0,5). In beiden Gruppen herrschte ein ausgewogenes Geschlechtsverhältnis (Tabelle 2) [27].

Tabelle 2: Beschreibung der Stichprobe [27]

\begin{tabular}{|l|c|c|c|c|}
\hline \multirow{2}{*}{ Gruppe } & \multicolumn{2}{|c|}{ Intervention (n=49) } & \multicolumn{2}{c|}{ Kontrolle (n=33) } \\
\cline { 2 - 5 } & Anzahl (n) & in \% & Anzahl (n) & in \% \\
\hline Mädchen & 23 & 46,9 & 17 & 51,2 \\
Jungen & 26 & 53,1 & 16 & 48,5 \\
\hline Gesamtstichprobe (n=82) & 49 & 59,8 & 33 & 40,2 \\
\hline
\end{tabular}

\section{Bewertung der Entdeckermodule}

In der Einzel-Bewertung der drei Module, vergaben die Schüler in der IG vornehmlich die Schulnoten „sehr gut“ und ,gut“ (Tabelle 3). Des Weiteren gaben die Schüler mehrheitlich an, dass ihnen die Entdeckermodule „sehr viel“ (85,7 \%) bzw. „ein bisschen“ (14,3\%) Freude bereitet haben. Negative Bewertungen in Form der Antworten „Nicht so“ oder „gar nicht“ wurden nicht vorgenommen [27].

Tabelle 3: Bewertung der Entdeckermodule durch die Schüler der IG [27]

\begin{tabular}{|l|l|l|}
\hline Entdeckermodule & Schulnote (Median) & $\begin{array}{l}\text { Schulnote } \\
\text { SD) }\end{array}$ \\
\hline Sandwichwettbewerb $(\mathrm{n}=42)$ & 1,0 & $1,4 \pm 0,7$ \\
\hline Lassi, Smoothie und Co. $(\mathrm{n}=45)$ & 1,0 & $1,5 \pm 0,8$ \\
\hline Lebensmittelquiz $(\mathrm{n}=46)$ & 2,0 & $1,9 \pm 0,7$ \\
\hline
\end{tabular}

\section{Erreichen der Zielgruppe und Auswirkungen}

Gut ein Drittel der Schüler gab an, durch die Module neue Lebensmittel kennengelernt zu haben. Ein Fünftel der Kinder aß diese Lebensmittel auch nach der Intervention. Fast die Hälfte der Kinder in der IG hat Rezepte der Module auch zu Hause zubereitet (Abbildung 2). 
Abbildung 2: Antworten der IG (T3) auf die Fragen "Hast du während der Entdeckungsreise Essen neue Lebensmittel kennen gelernt?", "Isst du jetzt andere Lebensmittel als vorher?", "Hast du etwas von den Rezepten der Entdeckungsreise Essen zu Hause zubereit?“ [27]

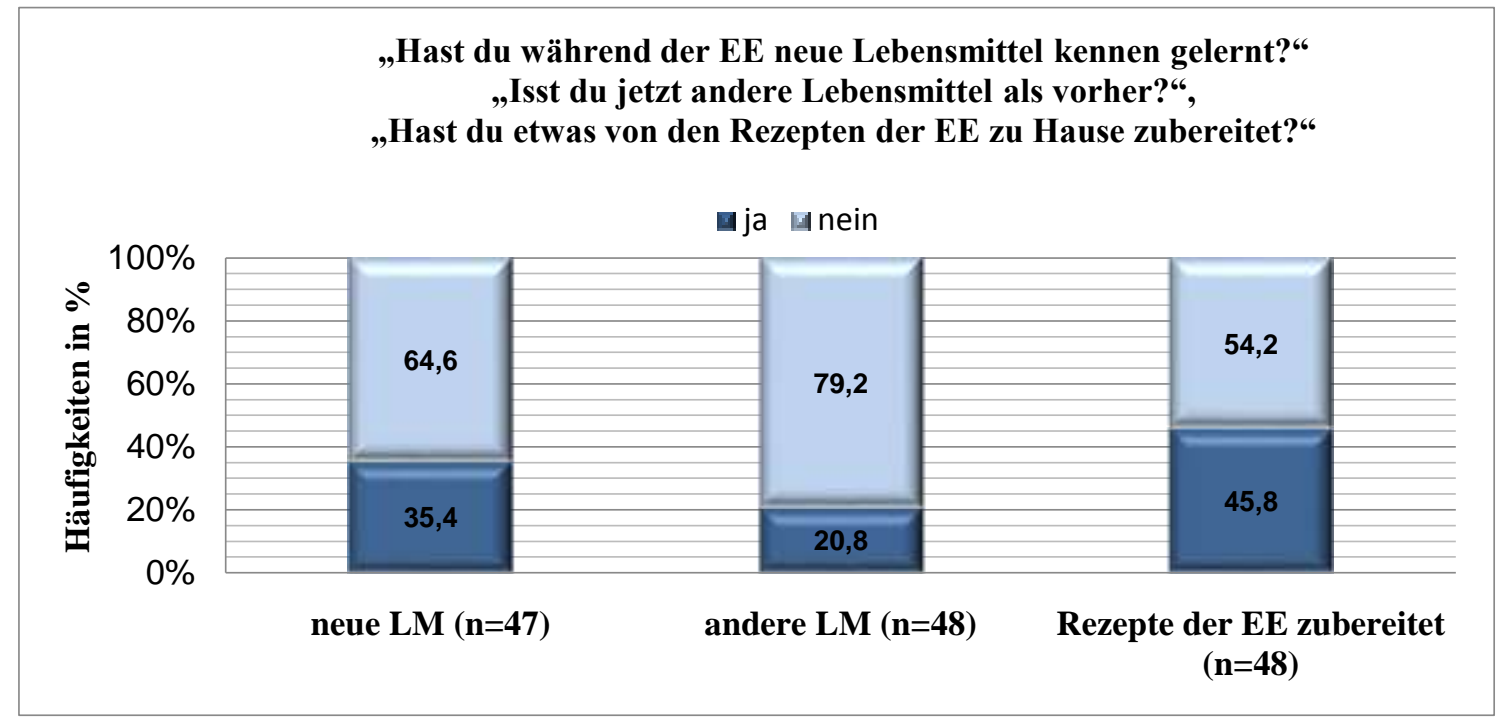

Der Anteil an Schülern in der IG, der die Aussage „Ich koche zu Hause“ mit „nie“ oder „selten“ beantwortete, sank nach der Interventionsphase um rund $20 \%$, während sich der Anteil an Kindern, die nach dem Projekt ,häufig“ zu Hause kochten (18,4\%), mehr als verdoppelt hat (37,5\%) (Abbildung 3). Auch in der Kontrollgruppe sank der Anteil an Schülern, die „nie“ oder „selten“ kochen um 15,1\% bzw. 3,1\%, während mehr als doppelt so viele Schüler nach Abschluss angaben, zu Hause „häufig“ zu kochen (36,4 \%). Die Differenz zwischen Pre- und Postbefragung war innerhalb der jeweiligen Untersuchungsgruppe signifikant (IG: $\mathrm{p}=0,000$; KG: $\mathrm{p}=0,013$ ). Der Vergleich beider Kollektive miteinander erbrachte keine signifikanten Unterschiede zwischen den Gruppen (Abbildung 3).

Die Ergebnisse der Kontrollfrage „Bereitest du dir Gerichte zu Hause selbst zu?“ zeigte keine signifikanten Veränderungen [27]. 

Essen"

Abbildung 3: Unterschiede der Antworten zu der Aussage „Ich koche zu Hause“ nach Gruppenzugehörigkeit und Befragungszeitpunkt $\Delta$ (Differenz zwischen Pre- und Postbefragung); Interventionsgruppe (nT0=49/nT3=48) $* * *(\mathrm{p}=0,000) ;$ Kontrollgruppe $(\mathrm{nT} 0=33 / \mathrm{nT} 3=32) *(\mathrm{p}=0,013)[27]$

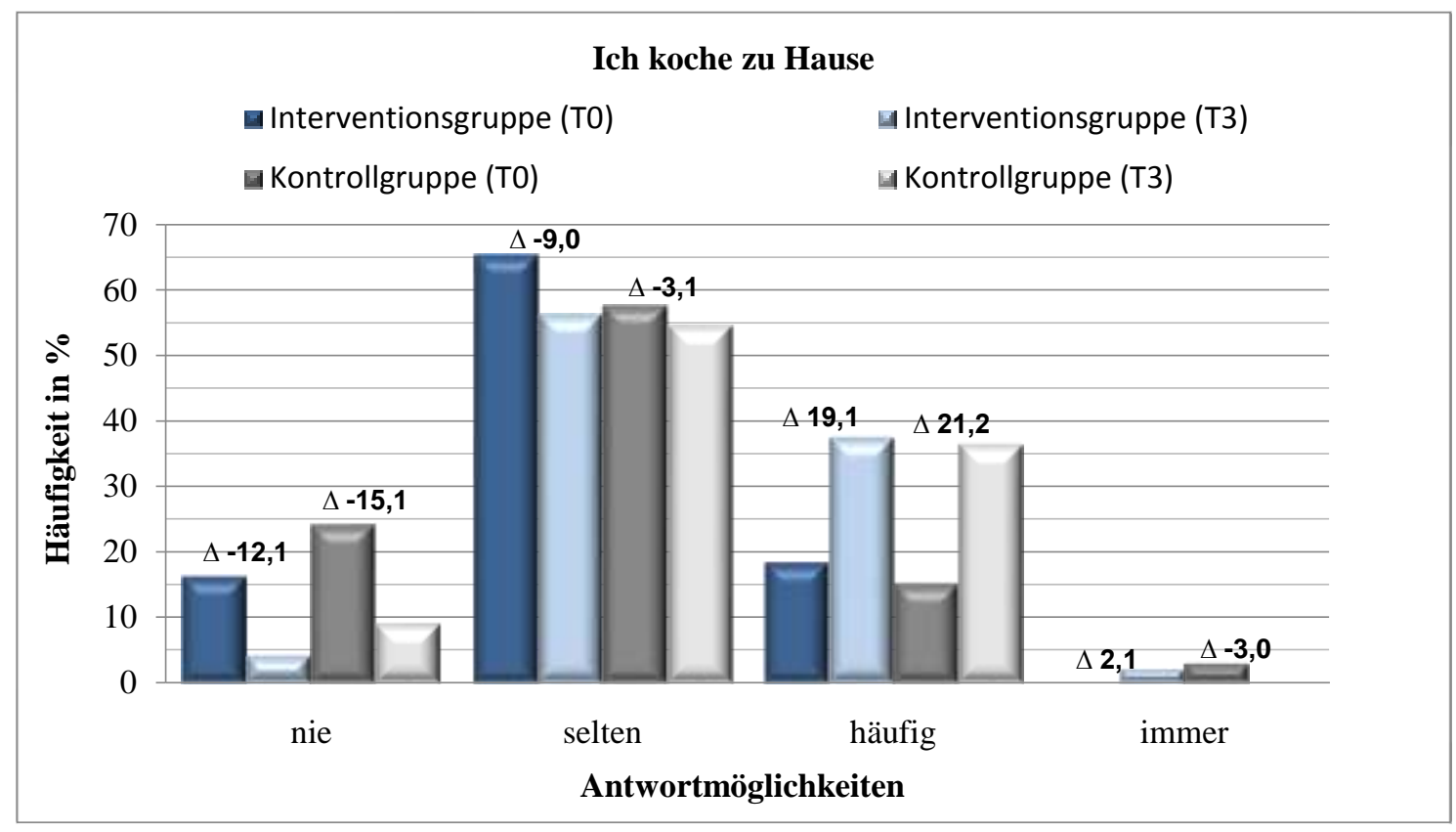

Auf die Frage „Machst du dir Gedanken darüber was du isst?“ überwog die Nennung „ein bisschen“ (Abbildung 4). In der IG zeigte sich eine deutliche Steigerung der Schüler, die sich „sehr“ viele Gedanken machen (16,7\% (T3) vs. 6,3\% (T0)).

Abbildung 4: Unterschiede in der Beantwortung der Frage „Machst du die Gedanken darüber was du isst?“" nach Gruppenzugehörigkeit und Befragungszeitpunkt $\Delta$ (Differenz zwischen Pre- und Postbefragung); Interventionsgruppe $(\mathrm{n}=48) *(\mathrm{p}=0,021)$; Kontrollgruppe $(\mathrm{nT} 0=32 / \mathrm{nT} 3=33)$ n. $\mathrm{s} .(\mathrm{p}=1,000)$ [27]

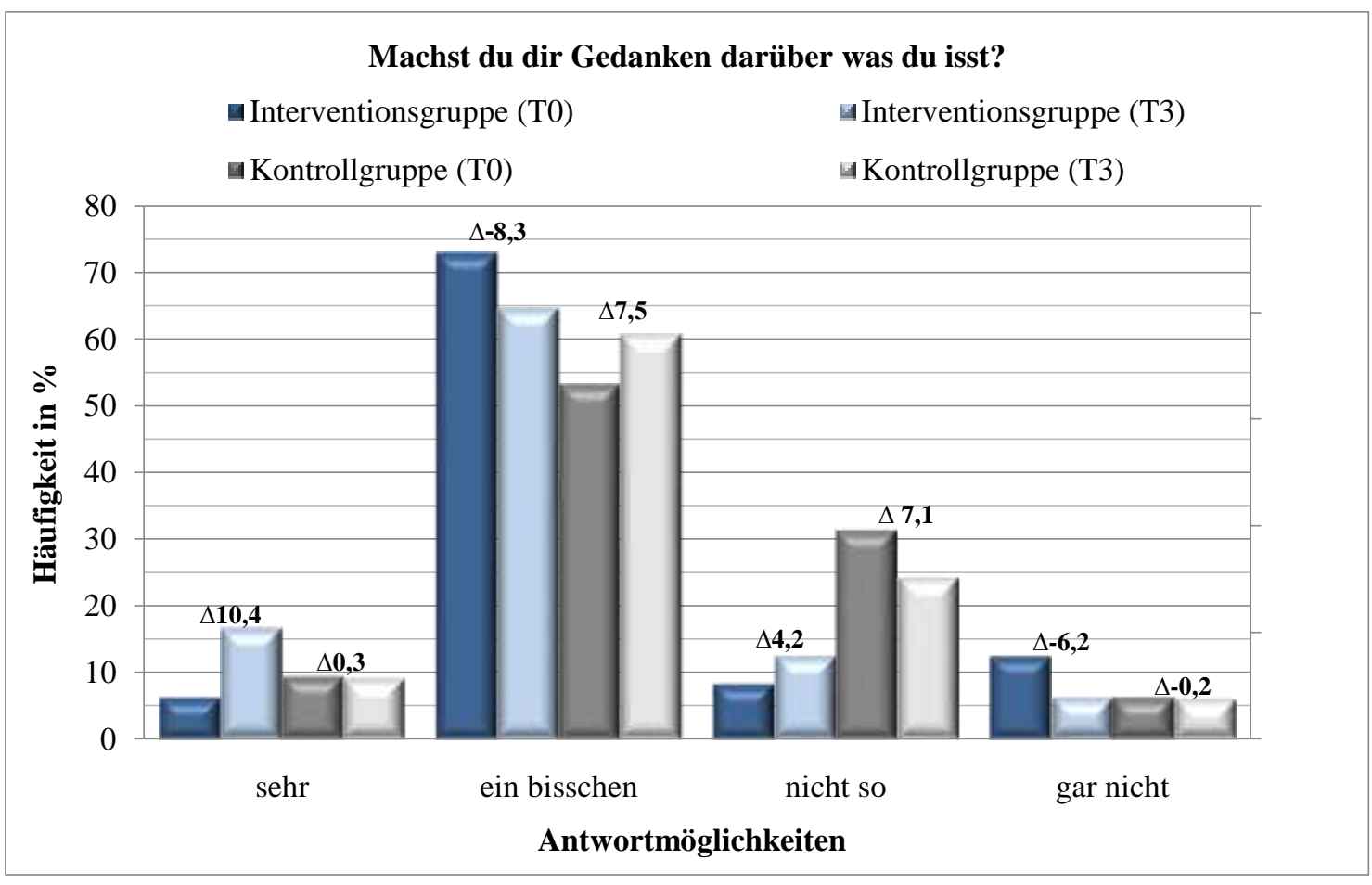




\section{Diskussion}

Der didaktische Ansatz des Projekts EE hat einen Schwerpunkt auf Handlungs-, Schüler- und Alltagsorientierung und entspricht damit aktuellen Erkenntnissen der Forschung, Zielgruppen in ihrer jeweiligen Lebenswelten zu erreichen [7]. Während der Entwicklung des Projekts zeigten sich bereits positive Effekte bei den Schülern der Entwicklungsschule. Die vorgestellte Untersuchung hatte zum Ziel, herauszufinden, ob auch einzelne ausgekoppelte Elemente im Schulalltag einer normalen Schule eingesetzt werden können und ob bereits bei vergleichsweise kurzzeitiger Intervention positive Veränderungen erzielt werden können.

Spaß, Freude und Erfolg sind entscheidende Grundlagen für operantes Konditionieren. Daher wurde anhand dieser Indikatoren im Schulnotensystem überprüft, ob die Schüler in ihrer Lebenswelt erreicht wurden. Weiterhin wurde überprüft, ob sich die Schüler nach der Intervention mehr Gedanken über ihr Essen machen. Auf Grund der positiven Bewertungen in der IG kann davon ausgegangen werden, dass die Schüler über die geprüften Module erreicht werden konnten (Hypothese 1).

Bei der Bewertung der Module durch die Schüler ist erkennbar, dass es einen Zusammenhang zwischen Attraktivität und Aktionslevel gibt. Mit zunehmender Partizipation und Praxisanteilen wurde die Attraktivität höher eingestuft (Hypothese 2). Allerdings steigt der logistische Aufwand für die Schulen meist mit zunehmenden Praxisanteilen, da u. a. Lebensmittel eingekauft und für den Moduleinsatz vorbereitet werden müssen.

Mehr als ein Drittel der Schüler in der IG hat während des Projekts neue Lebensmittel kennengelernt. Zudem gab fast die Hälfte der Schüler in der IG an, Rezepte der Entdeckermodule zu Hause nachgemacht zu haben. Am häufigsten wurde zu Hause ein Sandwich zubereitet, gefolgt von einem Smoothie. Dies lässt darauf schließen, dass die Module und auch die Auswahl der Rezepte für die Schüler einen Alltagsbezug aufweisen und die Schüler entweder bereits vor der Maßnahme die nötigen Kompetenzen besaßen oder diese durch die Module aufgebaut haben.

Einige Schüler gaben an, nach dem Projekt andere Lebensmittel als vorher zu essen. Das Projekt hat hier zu einer Erweiterung des Lebensmittelspektrums beigetragen. Daraus können jedoch keine Schlüsse gezogen werden, was dies für die Schüler im Alltag konkret bedeutet. Der Alltagsübertrag ist von der Verfügbarkeit und Verwendung verschiedener Lebensmittel im Haushalt der Familie abhängig. Familiäre Ernährungsgewohnheiten sind stark kulturell determiniert, werden aber auch durch das Ausmaß fester Mahlzeitenstrukturen und die finanzielle Situation in der Familie mitbestimmt. Diese deutlich maßgeblicheren Faktoren 
könnten eine Erklärung dafür sein, warum vier Fünftel der Schüler keine neuen Lebensmittel zu Hause gegessen haben. Einige Schüler gaben am Ende des Projekts an, zu Hause häufiger (mit) zu kochen. Dies kann als Zeichen zunehmender Wertschätzung von Lebensmitteln und gemeinsamen Mahlzeiten gewertet werden.

Hinsichtlich der Häufigkeit des selbstständigen Kochens zu Hause sind deutlich positive Veränderungen erkennbar. Der Anteil der Kinder, die nie oder selten zu Hause kochen sank, während sich der Anteil häufig zu Hause kochender Kinder mehr als verdoppelte (Abbildung 3). Allerdings konnten diese Effekte statistisch nicht gegen die Veränderungen in der Kontrollgruppe abgesichert werden. Die Kontrollgruppe erhielt im Interventionszeitraum Regel-Hauswirtschaftsunterricht und damit auch eine Intervention in ähnliche Richtung. Es handelte sich also nicht um eine klassische Kontrollbedingung. Sowohl die geprüften Module des Projekts EE wie auch der Regel-Hauswirtschaftsunterricht induzierten positive Veränderungen. In Detailaspekten schnitten die Module des Projekts jedoch etwas besser ab (Abbildung 3, Abbildung 4).

Die Ergebnisse zeigen in der Summe, dass der im Projekt gewählte Weg der Ernährungsbildung erfolgsversprechend und effizient ist. Partizipation, positives Erleben und Kompetenzerwerb sind Schlüsselfaktoren. Bisweilen werden in Projekten zur Ernährungsbildung jedoch die besonderen Rahmenbedingungen des Settings Schule vernachlässigt. Die einfache Umsetzbarkeit für Schulen und das aktive Einbeziehen der Lehrer sind zwei elementare Erfolgsfaktoren für Ernährungsbildung und deren nachhaltige Effekte. Die Attraktivität von erlebnis- und handlungsorientierten Ernährungsbildungs-Programmen bei Schülern und Lehrern hängt maßgeblich von den schulischen Ressourcen ab. So darf weder der personelle noch der zeitliche Aufwand zu hoch werden, was in diesem Beispiel u. a. durch die Integrierbarkeit der EE-Module in den Regelunterricht gegeben ist. Eine hohe inhaltliche und methodische Flexibilität ist aufgrund der stark variierenden strukturellen Voraussetzungen sowie der regionalen Unterschiede in den Lehrplänen notwendig für die Adaptation und Integration in das schulische Bildungsangebot.

\section{Schlussfolgerung}

Nicht alle Familien leben ihren Kindern im häuslichen Rahmen ein gesundes Ernährungsverhalten vor. Es gibt erhebliche soziale Unterschiede. Aus diesem Grund wird ein stärkeres Zusammenwirken aller Sozialisationsinstanzen (Familie, Kindertageseinrichtung, 
Schule, Quartier) für ein gesundes Aufwachsen gefordert [28]. Das Potential eines SettingAnsatzes besteht darin, alle Kinder - auch aus sozial schwachen Familien und unabhängig von der elterlichen Bereitschaft - einzubeziehen und etwaige soziale Schranken zu überwinden. Im schulischen Kontext erscheint der erlebnis-, schüler- und handlungsorientierte Ansatz des Ernährungsbildungs-Projekts „Entdeckungsreise Essen“ aufgrund der positiven Rückmeldungen der Schüler vielversprechend. Er berücksichtigt schulische Rahmenbedingungen und stellt Schulen ressourcensparende und flexibel einsetzbare Module zur Verfügung. Der praktische und partizipative Ansatz wird von den Schülern erwartungsgemäß positiv aufgenommen. Ein explorierender, praxis- und erlebnisorientierter Ansatz kann das Interesse von Schülern für die Themenfelder Ernährung, Zubereitung von Mahlzeiten, Esskultur und Wertschätzung fördern [29]. Insgesamt ist die Wirkung einzelner Module jedoch geringer als die Umsetzung eines Gesamtkonzeptes. Damit ist grundsätzlich ein vollständiger Einsatz (hier: Erlebniskochen plus alle Entdeckermodule) dem Auskoppeln einzelner Module vorzuziehen. Allerdings zeigen auch ausgekoppelte Module einen positiven Effekt.

Es wäre ohne Frage wünschenswert, wenn zeitgemäße Ernährungsbildung nicht allein im Projektrahmen, sondern strukturell und kontinuierlich im Schulalltag verankert würde und so dem Beispiel aus Schleswig-Holstein folgt. Im Idealfall wird das Ernährungsbildungskonzept synergistisch mit einer attraktiven und gesundheitsfördernden Schulverpflegung kombiniert, z.B. nach den Qualitätsstandards der Deutschen Gesellschaft für Ernährung [30].

Um abschließende Aussagen über tatsächliche Änderungen z.B. im Ernährungsverhalten treffen zu können, bedarf es weiterer Untersuchungen mit einem Monitoring des tatsächlichen Essverhaltens, bzw. der Erhebung von Biomarkern. Künftige Studien sollten auch die Umsetzbarkeit in verschiedenen Schulformen und die Bewertung durch Lehrkräfte einschließen. 


\section{Literatur}

1. Büning-Fesel M (2008): Ernährungskompetenz ist Lebenskompetenz. Abstract des Vortrages vom 7. Mai 2008, 11. aid-Forum Du isst, wie du bist?. URL: http://ernaehrungsdenkwerkstatt.de/fileadmin/user_upload/EDWText/TextElemente/E rnaehrungskommunikation/aid_forum_2008_abstract_buening_fesel.pdf Zugriff: 15.05.2015.

2. Wippermann P, Krüger J (Hrsg.) (2014) Werte-Index 2014. 1. Auflage, Deutscher Fachverlag GmbH, Frankfurt am Main.

3. Hölling H, Schlack R (2007) Essstörungen im Kindes- und Jugendalter. Erste Ergebnisse aus dem Kinder- und Jugendgesundheitssurvey (KiGGS). Bundesgesundheitsbl - Gesundheitsforsch - Gesundheitsschutz 50:794-799.

4. Kurth BM, Schaffarth Rosario A (2010) Übergewicht und Adipositas bei Kindern und Jugendlichen in Deutschland. Bundesgesundheitsbl, 53: 643-652.

5. Gerhards J, Rössel J (2003) Das Ernährungsverhalten Jugendlicher im Kontext ihrer Lebensstile - Eine empirische Studie, Forschung und Praxis der Gesundheitsförderung, Band 20, Bundeszentrale für gesundheitliche Aufklärung, Köln.

6. World Health Organization (WHO) (2003), Diet, Nutrition And The Prevention Of Chronic Diseases: Report of a Joint WHO/FAO Expert Consultation, World Health Organization, Geneva, WHO Technical Report Series No. 916.

7. Bartsch S et al. (2013) Ernährungsbildung - Standort und Perspektiven. Ernährungs Umschau 2/2013. M84-M94.

8. Philipps U (2004) Evaluation gesundheitsfördernder Maßnahmen bezüglich des Ernährungsverhaltens von Grundschulkindern. Bad Heilbronn: Verlag Julius Klinkhardt. [Dissertation].

9. Bundesministerium für Familie, Senioren, Frauen und Jugend (BMFSFJ) (Hrsg.) (2005) Zwölfter Kinder und Jugendbericht. Bericht über die Lebenssituation junger Menschen und die Leistungen der Kinder und Jugendhilfe in Deutschland. URL: http://www.bmfsfj.de/doku/Publikationen/kjb/data/download/kjb_060228_ak3.pdf Zugriff: 05.01.2015. 
10. Molderings M (2007) Evaluation pädagogisch-didaktischer Ansätze im Rahmen der gesundheitsförderlichen Ernährungserziehung der Grundschule: Einfluss von Unterrichtsformen, Elterneinbeziehung und Zielvereinbarung hinsichtlich einer Veränderung des Ernährungsverhaltens. Baltmannsweiler: Schneider Verlag Hohengehren. [Dissertation].

11. Bayrisches Staatsministerium für Unterricht und Kultus (STMUK) (Hrsg.) Gesunde Schüler lernen besser. URL: http://www.km.bayern.de/ministerium/schule-undausbildung/erziehung/gesundheitsfoerderung.html Zugriff: 05.01.2015.

12. Bartsch S (2009) Einflüsse auf das Essverhalten Jugendlicher. In: Kinderernährung aktuell. Schwerpunkte für Gesundheitsförderung und Prävention. 92-101.

13. Methfessel B (2007) Zwischen "Core Needs" und "Convenience" - Pädagogische Einflussmöglichkeiten auf die Lebensmittelauswahl und Ernährung der Zukunft. Ernährungs Umschau 54: 378-383.

14. Methfessel B (2008) Salutogenese - Ein Modell fordert zum Umdenken heraus. Teil 2 Herausforderungen für die Gesundheitsförderung. Ernährungs Umschau 55: 37-43.

15. HEINDL I (2009) Ernährungsbildung - curriculare Entwicklung und institutionelle Verantwortung. Ernährungs Umschau 56: 578-573.

16. Heindl I (2003) Studienbuch Ernährungsbildung. Ein europäisches Konzept zur schulischen Gesundheitsförderung. Bad Heilbrunn: Verlag Julius Klinkhardt.

17. Auswertungs- und Informationsdienst für Ernährung, Landwirtschaft, Verbraucherschutz e. V. (AID) (Hrsg.) Ernährungs- und Verbraucherbildung. URL: http://www.aid.de/lernen/ernaehrungs_verbraucherbildung.php Zugriff: 10.11.2014.

18. Auswertungs- und Informationsdienst für Ernährung, Landwirtschaft, Verbraucherschutz e. V. (AID) (Hrsg.) SchmExperten - Ernährungsbildung an weiterführenden Schulen. URL: http://www.aid.de/lernen/schmexperten.php Zugriff: 05.01.2015.

19. Heseker H et al. (2007) Schlussbericht des Modellprojekts „Reform der Ernährungsund Verbraucherbildung in Schulen (REVIS)“. Paderborn. URL: https://dsg.unipaderborn.de/fileadmin/evb/forschung_und_entwicklung/REVIS/REVIS-

Schlussbericht-mit_Anhang.pdf Zugriff: 10.11.2014.

20. Heindl I (2010) Reform der Ernährungs- und Verbraucherbildung an Schulen Vorreiterrolle Schleswig-Holsteins im Bundesgebiet -. URL: http://www.uniflensburg.de/ihl/downloads/Verbraucherbildung.pdf Zugriff: 27.06.2015. 
21. Ellrott T, Ranke C, Wagner N (o. J.): Entdeckungsreise Essen. Ein praxisnahes Schulkonzept zur Ernährungsbildung. URL: http://www.pebonline.de/uploads/tx_ ernaehrungundbewegung/EntdeckungsreiseEssen_LowRes.pdf Zugriff: 27.06.2015.

22. Niedersächsisches Kultusministerium (Hrsg.) (2010) Kerncurriculum für die Integrierte Gesamtschule Schuljahrgänge 5 - 10. Arbeit-Wirtschaft-Technik. URL: http://db2.nibis.de/1db/cuvo/datei/kc_awt_igs_internet_august_2010.pdf Zugriff: 05.01.2015.

23. Wagner N (2010): Entdeckungsreise Essen. Ein praxisnahes Schulkonzept zur Ernährungsbildung. Folienvortrag. 18. Aachener Diätetik-Fortbildungsveranstaltung des Verbandes für Ernährung und Diätetik (VFED) e. V. 10.-12.09.2010.

24. Lichtenstein S, Teufel U, Weiland C et al. (2011) Adipositasprävention in Grundschulen. Monatsschr Kinderheilkd. 159: 751-757.

25. Ellrott T, Barlovic I (2012): Einflussfaktoren auf das Essverhalten von Kindern und Jugendlichen. Kinderärztliche Praxis 83, 213-217

26. Deutsche Gesellschaft für Ernährung e.V. (2008): Stellungnahme zur erweiterten Nährwertinformation auf der Basis des „1 plus 4“ - Modells. September 2008; www.dge.de

27. Schöberle H (2011) Evaluation einer modulbasierten Schulintervention zur praktischen Ernährungsbildung am Beispiel des Projekts „Entdeckungsreise Essen“ des Instituts für Ernährungspsychologie an der Universitätsmedizin Göttingen.

28. Fröschl B, Haas S, Wirl C (2009) Prävention von Adipositas bei Kindern und Jugendlichen (Verhalten- und Verhältnisprävention). Schriftenreihe Health Technology Assessment (HTA) in der Bundesrepublik Deutschland. Deutsches Institut für Medizinische Dokumentation und Information (DIMDI) (Hrsg.). Köln. URL: http://portal.dimdi.de/de/hta/hta_berichte/hta242_bericht_de.pdf Zugriff: 20.11.2014.

29. Ellrott T (2013): Perspektiven der schulischen Ernährungsbildung. VFED Sonderheft „Vitamine Schulkantine: Gute Verpflegung in Kitas und Schulen“, 59-60.

30. Deutsche Gesellschaft für Ernährung: DGE-Qualitätsstandard für die Schulverpflegung, 4. Auflage 2014. URL: http://www.schuleplusessen.de/fileadmin/user_upload/ Bilder/DGE_QS_Schule_Essen_web.pdf Zugriff: 23.04.2015. 


\section{Abschlussbetrachtung}

Der erste Beitrag thematisiert die Evaluation des Projektes „5 am Tag für Kids“ der Niedersächsischen Krebsgesellschaft e.V. Mit Hilfe eines Verzehrshäufigkeitsfragebogens wurde das Essverhaltens der an der Intervention teilnehmenden Dritt- und Viertklässler (1.376 Kinder) vor sowie kurz- und mittelfristig nach der Projektdurchführung erfasst. Auf Basis dieser Ergebnisse lässt sich schlussfolgern, dass diese kurze, überwiegend auf Wissensvermittlung ausgerichtete Intervention keine nachhaltigen Effekte auf das Essverhalten, insbesondere auf den Obst- und Gemüseverzehr der Schülerinnen und Schüler zeigt. Bei der Ergebnisinterpretation sind allerdings mögliche Fehlerquellen, die aus der Wahl der Erhebungsmethode oder aus dem gewählten Studiendesign resultieren, zu berücksichtigen. Da die gewünschten Verhaltensänderungen nicht erzielt wurden, muss auch geschlussfolgert werden, dass das übergeordnete Ziel durch eine derartige Präventionsmaßnahme langfristig das Auftreten chronischer Erkrankungen wie Krebs zu beeinflussen, auf diesem Weg nicht erreicht werden kann. Die Ergebnisse verdeutlichen allerdings auch, wie wichtig es ist, die im Setting Schule eingesetzten verhaltens- und verhältnispräventiven Maßnahmen auf ihre Wirksamkeit hin zu untersuchen, um diese im Hinblick auf ihr Kosten-Nutzen-Verhältnis bewerten zu können.

Schon Volker Pudel, der Nestor der Ernährungspsychologie in Deutschland, hat in Frage gestellt, inwieweit Verhaltensprävention, die hauptsächlich auf Informationsvermittlung basiert, um das Wissen und die Einstellungen des Individuums zu ändern, in der Lage ist, emotionales Verhalten zu beeinflussen (Pudel, 2006). Er hält Verhaltensprävention nur für sinnvoll, „wenn sie das Essverhalten als emotionales Verhalten begreift und Methoden anwendet, die geeignet sind, auf Emotionen einzuwirken“ (Pudel, 2006, S. 96). Aus diesem Grund wird der Verhältnisprävention zunehmend Bedeutung beigemessen, zumal Wirksamkeitsanalysen von Projekten zeigen, dass Konzepte zur Gesundheitsförderung, die die beteiligten Settings ändern, oft dauerhafte Wirkungen zeigen (Heindl, 2009). Bezogen auf die Verbesserung des kindlichen Essverhaltens bedeutet dies, entsprechend konzipierte Programme in Erziehungs- und Bildungseinrichtungen zu implementieren (Heindl, 2009) mit dem Ziel, allen Kindern und Jugendlichen das nötige Wissen und die praktischen Fähigkeiten bezogen auf den Einkauf, die Vor- und Zubereitung sowie den Genuss gesundheitsförderlicher Lebensmittel und Speisen zu vermitteln (Mikkelsen, 2005). Auch durch optimierte Angebote von Speisen und Getränken in Kantinen, im Kiosk oder Snack-Automaten können Kindertagesstätten und Ganztagsschulen, langfristig die Lebensmittelauswahl bzw. - 
präferenzen der Kinder beeinflussen (Pudel, 2006). Dabei ist darauf zu achten, dass die im Unterricht vermittelten Inhalte Hand in Hand gehen mit dem Lebensmittelangebot bzw. der Schulverpflegung (Mikkelsen, 2005). In diesem Kontext spielen die beteiligten Lehrkräfte eine wichtige Rolle. Sie sind zum einen Vorbild für die Kinder, zum anderen gestalten sie den Unterricht und die Pausenverpflegung. Sie entscheiden, welche Unterrichtsinhalte schwerpunktmäßig behandelt und welche zusätzlichen Projekte im Bereich „gesunde Ernährung“ integriert werden. Durch die Identifikation der Lehrkräfte mit moderner Ernährungsbildung können auch sie dazu beitragen, eine schulische Umgebung zu schaffen, die Kindern entsprechende Ernährungskompetenzen vermittelt und die erforderlichen Rahmenbedingungen hinsichtlich eines Angebots von gesundheitsförderlichen Lebensmitteln schafft.

An diesen Punkt knüpfen die Inhalte und Erkenntnisse des zweiten Beitrags an, die darlegen, dass Kinder die Lebensmittel bevorzugen, die sie häufig angeboten bekommen. Die Verzehrmengen von Obst und Gemüse fallen signifikant höher bei der Gruppe von Kindern aus, die im Elternhaus und für die Pausenverpflegung Obst und Gemüse zur Verfügung gestellt bekommen. Dieser Aspekt fällt in den Verantwortungsbereich der Eltern.

Da die Schülerinnen und Schüler durch den Ausbau von Ganztagsschulen zunehmend mehr Zeit in der Schule verbringen und dort auch mindestens eine Mahlzeit verzehren (Philipps, 2004), kommt dem Lebensmittelangebot in Bildungsinstitution vermehrt Bedeutung zu. Kindertageseinrichtungen und Schulen sind aufgefordert, ein entsprechendes Angebot an gesundheitsförderlichen Snacks, Speisen und Getränken bereitzustellen. Zum einen steht hier die Sicherstellung einer qualitativ hochwertigen Mittagsverpflegung im Fokus, zum anderen aber auch das von der EU-Kommission initiierte europäische Schulfruchtprogramm. Mit dem Angebot eines flächenweiten kostenfreien oder auch kostenpflichtigen Schulfruchtprogramms ließe sich der Obst- und Gemüseverzehr der Kinder steigern. Eine Übersicht über eingeführte Schulfruchtprogramme bzw. deren Pilotprojekte in Europa bestätigen, dass sie das Potential haben, Essgewohnheiten langfristig $\mathrm{zu}$ verändern. Alle Untersuchungen wiesen eine signifikante Steigerung des Obst- und / oder Gemüsekonsums in der Interventionsgruppe auf (Kaiser und Schönberger, 2008). Auch eine erste Evaluation in den am EU-Schulobstprogramm teilnehmenden Bundesländern in Deutschland für das Schuljahr 2010-2011 verzeichnete eine Zunahme des Verzehrs (Bundesministerium für Ernährung, Landwirtschaft und Verbraucherschutz). Durch ein entsprechendes Angebot ließen sich möglicherweise soziale Ungleichheiten oder Defizite in der elterlichen Versorgung kompensieren. 
Häufig fehlt den beteiligten Personen wie Schulleitern, Cafeteria-Personal oder KioskBetreibern jedoch das erforderliche Fachwissen, um die erwünschten Veränderungen bei der Schulverpflegung $\mathrm{zu}$ initiieren. Hier sollten die Bundesländer dafür Sorge tragen, Bildungsinstitutionen bei der Implementierung durch entsprechend geschultes Fachpersonal zu unterstützen. Durch Einrichtung der landesweiten Vernetzungsstellen Schulverpflegung wurde dem bereits Rechnung getragen.

Bleibt noch die Frage, inwieweit es tatsächlich gelingen kann, dass Erfahrungen, die die Kinder durch verhaltens- und verhältnispräventive Maßnahmen in Bildungseinrichtungen erleben, auch die Lebensmittelauswahl und Zubereitung von Speisen in der häuslichen Umgebung beeinflussen (Pudel, 2006). Es gibt jedoch Hinweise darauf, dass Eltern durch ihre Kinder erreicht werden können (Perry et al., 1989). Beispielsweise deuten die Befragungsergebnisse im dritten Beitrag „Praktische Ernährungsbildung in der 5./6. Klasse: Evaluation des Projekts 'Entdeckungsreise Essen'“ darauf hin, dass sich durch partizipative, teilhabende Interventionsansätze in der Schule auch Veränderungen in der häuslichen Umgebung der Kinder hervorrufen lassen. Das Konzept ist handlungs-, schüler- und alltagsorientiert und darauf ausgerichtet, den Kindern Kompetenzen zu vermitteln, die sie in ihrer Lebenswelt anwenden können. Bei einigen Schülern erweiterte sich nach der Teilnahme an den Modulen das Lebensmittelspektrum und auch bei der Häufigkeit des selbstständigen Kochens bzw. der Zubereitung von projekteigenen Rezepten zu Hause waren positive Veränderungen zu verzeichnen.

\section{Limitationen}

Abschließend werden einige Limitationen der im Rahmen dieser Arbeit durchgeführten Interventionsstudie aufgeführt. Diese Einschränkungen betreffen die eingesetzten Datenerhebungsinstrumente, andere die organisatorische Vorgehensweise bei der Befragung. Die für die Erfassung des Essverhaltens gewählte Methode des Verzehrshäufigkeitsfragebogens beinhaltete Fehlerquellen. Zum einen wurden nur Schätzmengen der verschiedenen Lebensmittelgruppen erfasst, zum anderen sind Eltern grundsätzlich keine verlässlichen Berichterstatter, da sie beispielsweise den Außer-Haus-Verzehr ihres Kindes nicht beobachten können. Darüber hinaus führte die Fokussierung des Elternfragebogens auf das Thema Obst und Gemüse vermutlich zum Phänomen der sozialen Erwünschtheit. Dieser Effekt würde die im Vergleich zu anderen Studien sehr hoch ausfallenden durchschnittlichen Verzehrmengen von Obst und Gemüse zumindest teilweise erklären. 
Die Bereitschaft den FFQ dreimalig auszufüllen, war bei den Eltern sehr gering, so dass zwar der Verzehrshäufigkeitsfragebogen vor der Intervention von vielen Kindern vorlag, häufig der zweite und / oder dritte FFQ nach dem Projekt aber nicht mehr zurückgegeben wurde, so dass die Entwicklung des Essverhaltens nicht beobachtet werden konnte. Die hohe dropout-Rate lässt sich gegebenenfalls auf sprachliche Barrieren bzw. Unverständnis über das mehrmalige Ausfüllen des gleichen Fragebogens sowie den damit verbundenen Zeitaufwand zurückführen. Vermutlich führte dies zu einem gewissen Selektionseffekt, bei dem sich vorwiegend Eltern, die großen Wert auf die Ernährung ihres Kindes legen und bei denen davon ausgegangen werden kann, dass die Kinder viel Obst und Gemüse zu sich nehmen, gewissenhaft an der Befragung beteiligt haben. Dieser Aspekt würde ebenfalls zur Erklärung der hohen Durchschnittswerte bezogen auf den Obst- und Gemüseverzehr beitragen.

Die Verteilung der unterschiedlichen Fragebögen zu den verschiedenen Zeitpunkten wurde durch die jeweilige Lehrkraft sichergestellt, was eine gewissenhafte Ausführung der Lehrkraft voraussetzte und zudem einen hohen organisatorischen Überwachungsaufwand mit sich brachte. Eine andere Möglichkeit, die Eltern zu erreichen gab es jedoch nicht.

Aufgrund des Projektdesigns und des daraus resultierenden Studiendesigns wurde auf die Installation eine Kontrollgruppe verzichtet.

Auch für den zweiten Beitrag, der sich mit den Einflussfaktoren auf den Obst- und Gemüseverzehr der Schülerinnen und Schüler beschäftigt, sind Limitationen zu nennen. Aufgrund des umfangreichen FFQs und der möglicherweise altersbedingten begrenzten Aussagefähigkeit der Kinder, wurde bei der Konzeptionierung von Eltern- und Schülerfragebögen darauf geachtet, die Anzahl der Fragen und Inhalte zu beschränken. Aus diesem Grund wurden weiterführende Aspekte $\mathrm{zu}$ Wissen, Einstellungen und Verhaltensabsichten rund um das Thema Ernährung bzw. Obst und Gemüse nur in geringem Umfang erfasst, so dass nur begrenzt Zusammenhänge zwischen Faktoren und dem kindlichen Verzehr dieser Lebensmittel herzustellen sind. Die Einflussfaktoren auf das Essverhalten und speziell auf den Obst- und Gemüsekonsum sind jedoch in zahlreichen Studien umfassend untersucht worden.

Darüber hinaus greift diese Arbeit im dritten Beitrag Ergebnisse aus der Untersuchung der Wirksamkeit ausgewählter Module der „Entdeckungsreise Essen“ als weitere praktische Maßnahme der Ernährungsbildung auf. In diesem Zusammenhang muss als Limitation aufgeführt werden, dass bei dieser Pilotstudie nicht das tatsächliche Ernährungsverhalten und 
deren potentielle Veränderung erfasst wurden. Stattdessen wurde lediglich aufgrund der Antworten auf Fragen wie „Isst du jetzt andere Lebensmittel als vorher?“, „Hast du etwas von den Rezepten der Ernährungsreise Essen zu Hause zubereitet?“ oder das vermehrte Kochen zu Hause auf eine Übertragung der erlernten Verhaltensweisen und Handlungskompetenzen auf die häusliche Umgebung geschlossen. Als weitere Limitation ist zu erwähnen, dass auch die Kontrollgruppe nicht ganz ohne Intervention geblieben ist, da die Kinder im Befragungszeitraum am Hauswirtschaftsunterricht teilgenommen haben, in dem u. a. auch Kocheinheiten durchgeführt wurden.

\section{Fazit}

Insgesamt besteht eine große Heterogenität bezogen auf die Ergebnisse von Studien, die „,5 am Tag"-Interventionen untersuchten. Basieren diese Interventionen auf einem verhaltens- und verhältnispräventivem Ansatz und beinhalten mehrere Komponenten wie beispielsweise Unterrichtscurriculum, Veränderungen im schulischen Speisenangebot (Mensa, Schulkiosk) und Einbezug der Eltern, konnten signifikante Steigerungen bezogen auf den Obst- und Gemüseverzehr der Kinder festgestellt werden (Knai et al., 2006; De Sa und Lock, 2007; Van Cauwenberghe et al., 2010). Auch das Bereitstellen von kostenfreiem Schulobst trägt zur Zunahme des Verzehrs dieser Lebensmittel bei (Bere et al., 2006; Bere et al., 2007). Grundsätzlich scheint es leichter zu sein, den Obstkonsum der Kinder positiv zu beeinflussen als den Gemüseverzehr (Evans et al., 2012). Inzwischen haben Studien herausgearbeitet, welche Elemente erfolgsversprechend sind und bei der Konzeptionierung von Interventionen berücksichtigt werden sollten:

- Maßnahmen basieren auf Theorien zur Verhaltensänderung,

- Verbesserung von Fertigkeiten zur Zubereitung von Speisen,

- ausreichender Zeitumfang und Intensität,

- Fokussierung auf Obst und Gemüse statt allgemeiner Ernährungsempfehlungen,

- Verbesserung der Verfügbarkeit von Obst und Gemüse,

- Einbezug der Eltern auch in die schulische Umgebung,

- angemessene Ausbildung der Lehrkräfte und des Kantinenpersonals

(Burchett, 2003; Klepp et al., 2005; Knai et al., 2006) 
Das Konzept für „5 am Tag für Kids“ wurde vor etwa 15 Jahren von einer Marketingagentur im Auftrag der Deutschen Krebsgesellschaft e.V. entwickelt. Zu dem Zeitpunkt war die Verhaltensforschung zum einen noch nicht so weit fortgeschritten, zum anderen basiert das Projekt vorwiegend auf Marketingstrategien und nicht auf Theorien zur Verhaltensänderung. Profilierung und Imagebildung des Lebensmittelhandels bei einer jungen Zielgruppe, Schaffung von Nähe zu potentiellen Kunden, Netzwerkbildung zwischen Lebensmittelhandel und Schulen durch eine neutrale Organisation (Landeskrebsgesellschaften) und Reduzierung von Berührungsängsten gegenüber Obst und Gemüse bei Kindern zur Absatzsteigerung standen im Vordergrund. Für die Durchführung waren folgende Aspekte relevant: Das Erreichen möglichst vieler Schulkinder landesweit, moderate Kosten, einfache Implementierung in den Schulen sowie ein geringer Arbeitsaufwand für die beteiligten Lehrkräfte. Aufgrund der Ergebnisse, dass eine kurze, wissensbasierte Intervention keine nachhaltigen Verhaltensveränderungen hervorruft, ist eine Neukonzeptionierung des Projektes unter Berücksichtigung der aktuellen Erkenntnisse aus der Forschung empfehlenswert, um die Effektivität der Maßnahme zu erhöhen. Basierend auf der Erkenntnis, dass das Bereitstellen von Obst und Gemüse einen entscheidenden Prädiktor für den Verzehr darstellt, wäre eine Modifikation des Konzeptes in Richtung Verbesserung des Angebotes anzustreben. Dies müsste unter Beteiligung aller betreffenden Landeskrebsgesellschaften und unter Beachtung finanzieller und personeller Ressourcen diskutiert werden.

Für die Gesamtbetrachtung bedeutet es, dass nach wie vor großer Handlungsbedarf bezüglich der Konzeptionierung und der Bündelung verhaltens- und verhältnispräventiver Maßnahmen zur Förderung eines gesunden Ernährungsverhaltens und damit der Gesunderhaltung der Bevölkerung besteht. Eingangs der Arbeit wurde der Zusammenhang zwischen Krebs und Lebensstilfaktoren wie Ernährung und Bewegung beschrieben und in diesem Kontext auch auf das große Präventionspotential, das eine Verbesserung dieser Faktoren, beinhaltet, hingewiesen. Hier ist die Regierung in Kooperation mit den jeweiligen Fachorganisationen aufgerufen, die entsprechenden Rahmenbedingungen zu gestalten, um evaluierte, effektive Präventionsmaßnahmen und -programme in geeigneten Settings wie Kindertageseinrichtungen und Schulen flächenweit zu installieren. Auf politischer Ebene wurde mit dem nationalen Aktionsplan „IN FORM - Deutschlands Initiative für gesunde Ernährung und mehr Bewegung“ bereits eine Basis geschaffen, um die Aktivitäten zu bündeln und mit der Umsetzung zu beginnen. Das in einigen Bundesländern eingeführte Schulfruchtprogramm trägt ebenfalls zur Verbesserung des institutionellen Angebotes gesundheitsförderlicher Lebensmittel bei. 
Darüber hinaus wurde mit der „Reform der Ernährungs- und Verbraucherbildung in Schulen“ (REVIS) der Grundstein für eine Ernährungswende in der Bildungspolitik gelegt. Das Kerncurriculum zur Ernährungsbildung umschreibt die Inhaltsfelder, in denen sich ein Kompetenzerwerb im Verlauf der Schulbildung vollziehen soll (Universität Paderborn, 2005). In Kombination mit einer gesteigerten Inanspruchnahme von Früherkennungsuntersuchungen könnten so die persönlichen, gesellschaftlichen und wirtschaftlichen Belastungen, die aus chronischen Erkrankungen wie Krebs, Herz-Kreislauferkrankungen und Diabetes resultieren, mit hoher Evidenz reduziert werden (Eyre et al., 2004). Hierfür wäre es allerdings erforderlich, dass sich auch die Ausrichtung der gesundheitlichen Versorgungssysteme in Richtung Prävention und Gesundheitsförderung verschiebt und Präventionsmaßnahmen kontinuierlich und nicht allein im Rahmen kurzer Projektphasen angeboten werden (Klotz et al., 2006). 


\section{Literatur}

Bere E., Veierød MB., Bjelland M. und Klepp KI. 2006. Free school fruit - sustained effect 1 year later. Health Education Research. Vol. 21, no. 2, 268-275.

Bere E., Veierød MB., Skare Ø. und Klepp KI. 2007. Free school fruit - sustained effect three years later. International Journal of Behavioral Nutrition and Physical Activity. 4:5.

Bundesministerium für Ernährung, Landwirtschaft und Verbraucherschutz (Hrsg.).

Zusammenfassung der Evaluationsergebnisse der in der Bundesrepublik Deutschland am EU-Schulobstprogramm teilnehmenden Länder für das Schuljahr 2010-2011. Berlin. URL: http://www.bmel.de/SharedDocs/Downloads/Ernaehrung/KitaSchule/EU-Schulfrucht_Evaluationsbericht.pdf?_blob=publicationFile (Zugriff am 17.06.2013).

Burchett H. 2003. Increasing fruit and vegetable consumption among British primary schoolchildren: a review. Health Education. Vol. 103, no. 2, 99-109.

De Sa J. und Lock K. 2007. School-based fruit and vegetables schemes: A review of the evidence. London School of Hygiene and Tropical Medicine. URL: http://www.5aday.co.nz/5adaywebsite/cms_resources/file/SCHOOL\%20REVIEW\%2 02007.pdf (Zugriff am 12.05.2011).

Evans CEL., Christian MS., Cleghorn CL., Greeenwood DC und Cade JE. 2012. Systematic review and meta-analysis of school-based interventions to improve daily fruit and vegetable intake in children aged 5 to 12 y. American Journal of Clinical Nutrition. 96, 889-901.

Eyre H., Kahn R. und Robertson RM. 2004. Preventing Cancer, Cardiovascular Disease, and Diabtes. Diabetes Care. Vol. 27, no. 7, 1812-1824.

Heindl I. 2009. Ernährungsbildung - curriculare Entwicklung und institutionelle Verantwortung. Ernährungs Umschau. 56, 568-573.

Kaiser J. und Schönberger G. 2008. Ein Schulfruchtprogramm für Deutschland. Ernährung im Fokus. 8-08, 278-286.

Klepp KI., Pereze-Rodrigo C., De Bourdeaudhuij I., Due P., Elmadfa I., Haraldsdottir J., Konug J., Sjostrom M., Thorsdottir I., Vaz de Almeida MD., Yngve A. und Brug J. 2005. Promoting Fruit and Vegetable Consumption among European Schoolchildren: Rationale, Conceptualization and Design of the Pro Children Project. Annals of Nutrion \& Metabolism. 49, 212-220.

Klotz T., Haisch J. und Hurrelmann K. 2006. Prävention und Gesundheitsförderung - Ziel ist anhaltend hohe Lebensqualität. Deutsches Ärzteblatt, Jg. 103, Heft 10, A 606-A 609. 
Knai C., Pomerleau J., Lock K. und McKee M. 2006. Getting children to eat more fruit and vegetables: A systematic review. Preventive Medicine. 85-95.

Mikkelsen BE., Barnekow Rasmussen V. und Young I. 2005. The role of school food service in promoting healthy eating at school - a perspective from an ad hoc group on nutrition in schools, Council of Europe. Food Service Technology. 5, 7-15.

Perry CL., Luepker RV., Murray DM., Hearn MD., Halper A., Dudovitz B., Maile MC. und Smyth M. 1989. Parent involvement with children's health promotion: A one-year follow-up of the Minnesota home team. Health Education Quarterly. 16, 171-180.

Philipps U. 2004. Evaluation gesundheitsfördernder Maßnahmen bezüglich des Ernährungsverhaltens von Grundschulkindern. Verlag Julius Klinkhardt, [Dissertation], Bad Heilbrunn.

Pudel V. 2006. Verhältnisprävention muss Verhaltensprävention ergänzen. Ernährungs Umschau. 53 (3). 95-98.

Universität Paderborn Fachgruppe Ernährung und Verbraucherbildung 2005. REVISModellprojekt. Reform der Ernährungs- und Verbraucherbildung in Schulen 20032005.

Van Cauwenberghe E., Maes L., Spittaels H., van Lenthe FJ., Brug J., Oppert JM. und De Bourdeaudhuij I. 2010. Effectiveness of school-based interventions in Europe to promote healthy nutrition in children and adolescents: systematic review of published and 'grey' literature. British Journal of Nutrition. 103, 781-797. 


\section{Abkürzungsverzeichnis}

DGE Deutsche Gesellschaft für Ernährung e.V.

DNS Desoxyribonukleinsäure

EKN Epidemiologisches Krebsregister Niedersachsen

EsKiMo Ernährungsstudie als KiGGS-Modul

EVS Einkommens- und Verbrauchsstichprobe

GEKID Die Gesellschaft der epidemiologischen Krebsregister in Deutschland e.V.

GKV Gesetzliche Krankenversicherung

KiGGS Kinder- und Jugendgesundheitssurvey, jetzt: Studie zur Gesundheit von Kindern und Jugendlichen in Deutschland

MRI Max Rubner-Institut

NVS I Nationale Verzehrsstudie I

NVS II Nationale Verzehrsstudie II

REVIS Reform der Ernährungs- und Verbraucherbildung in Schulen

RKI Robert Koch-Institut 


\title{
Danksagung
}

\author{
„Nur wer sein Ziel kennt, findet seinen Weg. “ \\ Epiktet, Philosoph, 50-125 n. Chr.
}

An dieser Stelle möchte ich mich bei allen bedanken, die zum Gelingen meiner Promotion beigetragen und mich in dieser Zeit begleitet haben.

Mein besonderer Dank geht an meinen Doktorvater, PD Dr. med. Thomas Ellrott, der mir mit vielen wertvollen Ratschlägen und Anregungen stets zur Seite gestanden hat. Bedanken möchte ich mich auch bei Anja Austel aus dem Institut für Ernährungspsychologie, die mir insbesondere bei statistischen Fragen immer eine große Hilfe war.

Mein tiefster Dank gebührt meinem Arbeitgeber, der Niedersächsischen Krebsgesellschaft e.V., der mir die Möglichkeit gegeben hat, mich im Rahmen meiner beruflichen Tätigkeit wissenschaftlich fortzubilden und diese Dissertation anzufertigen.

Bedanken möchte ich mich auch bei allen Schulleitern, Lehrkräften, Schülern und Eltern, die sich bereit erklärt haben an der Befragung teilzunehmen.

Mein größter Dank geht an meine Familie, die in jeder Lebenslage für mich da ist und immer an mich geglaubt hat, dass ich meinen Weg gehe. 


\section{Eidesstattliche Erklärung}

1. Hiermit erkläre ich, dass diese Arbeit weder in gleicher noch in ähnlicher Form bereits anderen Prüfungsbehörden vorgelegen hat.

Weiter erkläre ich, dass ich mich an keiner anderen Hochschule um einen Doktorgrad beworben habe.

Göttingen, den 15. September 2015

Unterschrift Silke Mittmann

2. Hiermit erkläre ich eidesstattlich, dass diese Dissertation selbständig und ohne unerlaubte Hilfe angefertigt wurde.

Göttingen, den 15. September 2015

Unterschrift Silke Mittmann 

